Pontifícia Universidade Católica

José Bruno Oliveira de Araújo

\title{
An Ultrawideband Metamaterial Absorber and a Parameter Retrieval Method
}

Dissertation presented to the Programa de Pós-graduação em Engenharia Elétrica of PUC-Rio in partial fulfillment of the requirements for the degree of Mestre em Engenharia Elétrica.

Advisor: Prof. Glaucio Lima Siqueira 


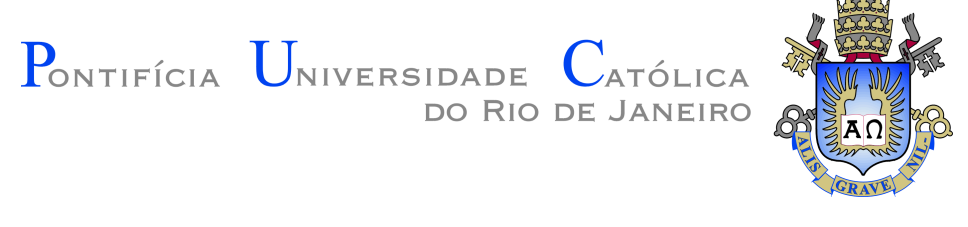

José Bruno Oliveira de Araújo

\section{An Ultrawideband Metamaterial Absorber and a Parameter Retrieval Method}

Dissertation presented to the Programa de Pós-graduação em Engenharia Elétrica of PUC-Rio in partial fulfillment of the requirements for the degree of Mestre em Engenharia Elétrica. Approved by the undersigned Examination Committee.

Prof. Emanoel Paiva de Oliveira Costa

Centro de Estudos em Telecomunicações - PUC-Rio

\section{Prof. Maurício Weber Benjó da Silva} Universidade Federal Fluminense - UFF

Dr. Jorge Ângelo Mitrione Souza

Centro de Estudos em Telecomunicações - PUC-Rio

Prof. Marbey Manhães Mosso

Centro de Estudos em Telecomunicações - PUC-Rio 
All rights reserved.

\section{José Bruno Oliveira de Araújo}

The author graduated in Telecommunication Engineering from the Universidade Federal Fluminense, in Niterói, Rio de Janeiro, Brazil, 2017.

Bibliographic data

de Araújo, José Bruno Oliveira

An Ultrawideband Metamaterial Absorber and a Parameter Retrieval Method / José Bruno Oliveira de Araújo; advisor: Glaucio Lima Siqueira . - Rio de janeiro: PUC-Rio, Departamento de Engenharia Elétrica, 2019.

v., 110 f: il. color. ; $30 \mathrm{~cm}$

Dissertação (mestrado) - Pontifícia Universidade Católica do Rio de Janeiro, Departamento de Engenharia Elétrica.

Inclui bibliografia

1. Engenharia Elétrica - Teses. 2. Engenharia Elétrica Teses. 3. Metamaterial;. 4. Absorvedor;. 5. Extração de Parâmetros;. 6. Banda Larga;. I. Siqueira, Glaucio Lima. II. Pontifícia Universidade Católica do Rio de Janeiro. Departamento de Engenharia Elétrica. III. Título. 


\section{Acknowledgments}

To begin with, I would like to thank my mother, Geilda Moura, for the support, love, and words of wisdom.

I would like to express my sincere gratitude to my advisor, Professor Glaucio Siqueira, for the enthusiasm, personality and guidance without which this journey would have been quite harder.

I also would like to thank Professor Marbey Mosso, for many discussions on electromagnetism and life, and also for the valuable advices.

I would like to thank Bianca Campinho, for the encouragement and unmeasurable contributions to this work.

I am grateful to the professors Cynthia Junqueira, Mauricio Weber and Erich Kemptner for their helpful contributions and many technical suggestions.

I would like to thank my lab colleagues and friends Julio Acosta, Yoiz Nuñez, and Johnes Ricardo for all support.

I thank PUC-Rio for the scholarship, financial support, and infrastructure granted to me.

This study was financed in part by the Coordenação de Aperfeiçoamento de Pessoal de Nível Superior - Brasil (CAPES) - Finance Code 001.

Finally, I would like to thank my family and friends for all support. 


\section{Abstract}

de Araújo, José Bruno Oliveira; Siqueira, Glaucio Lima (Advisor). An Ultrawideband Metamaterial Absorber and a Parameter Retrieval Method. Rio de Janeiro, 2019. 110p. Dissertação de mestrado - Departamento de Engenharia Elétrica, Pontifícia Universidade Católica do Rio de Janeiro.

This work presents the design, characterization, and measurement of an ultrathin and ultrawideband metamaterial absorber based on periodicallyarranged metallic square spiral and its equivalent circuit is proposed to provide a theoretical insight. The parameters of the equivalent circuit are extracted using an algorithm based on the least-square method and using a straightforward approach, which can be applied to broadband absorbers in general. The mechanism of absorption of the proposed structure is discussed and detailed. One of the simulated results showed an absorptivity of more than $90 \%$ from 11.4 to $20.0 \mathrm{GHz}$, covering the Ku band for TM as well as TE polarization, and this broadband feature is confirmed by the experimental measurement. Furthermore, the proposed metamaterial absorber is $\lambda / 16.4$ thick at the lowest frequency of absorption and presents angle stability up to $50^{\circ}$. In addition, the usage of the proposed absorber to the radar crosssection reduction in microstrip antennas is investigated and reductions up to $12.8 \mathrm{~dB}$ are achieved, and an absorber wall is presented to decrease the mutual coupling in antenna arrays.

\section{Keywords}

Metamaterial; Absorber; Parameter Retrieval; Broadband; 


\section{Resumo}

de Araújo, José Bruno Oliveira; Siqueira, Glaucio Lima. Absorvedor com Banda Ultra Larga Baseado em Estruturas Metamateriais e o Método de Extração de Parâmetros. Rio de Janeiro, 2019. 110p. Dissertação de Mestrado - Departamento de Engenharia Elétrica, Pontifícia Universidade Católica do Rio de Janeiro.

Este trabalho apresenta a idealização, caracterização e medições de um absorvedor metamaterial ultrafino com uma banda ultra larga, baseado em espiras quadradas arranjadas periodicamente. O circuito equivalente dessa estrutura é proposto para fornecer uma maior compreensão teórica. Os parâmetros desse circuito são extraídos utilizando um algoritmo baseado no método dos mínimos quadrados, usando uma abordagem direta, que pode ser aplicada aos absorvedores com banda larga em geral. O mecanismo de absorção da estrutura proposta é discutido e detalhado. Um dos resultados da simulação mostrou uma absortividade acima de $90 \%$ de 11.4 até $20 \mathrm{GHz}$, cobrindo a banda $\mathrm{Ku}$ inteiramente tanto para a polarização TM quanto para a TE, e essa banda larga é confirmada pelas medições experimentais. Além disso, a espessura do absorvedor metamaterial proposto é $\lambda / 16.4$, considerando o maior comprimento de onda da banda de operação, e apresenta estabilidade angular até $50^{\circ}$. Ademais, o uso do absorvedor proposto para redução da seção transversal de radar em antenas de microfita é investigado e reduções até 12.8 dB são obtidas, e uma parede absorvedora é apresentada para diminuição do acoplamento mútuo em conjuntos de antenas.

\section{Palavras-chave}

Metamaterial; Absorvedor; Extração de Parâmetros; Banda Larga; 


\section{Table of contents}

$\begin{array}{lr}\text { List of figures } & 9\end{array}$

$\begin{array}{ll}\text { List of tables } & 12\end{array}$

$\begin{array}{llr}1 & \text { Introduction } & 15\end{array}$

2 Theoretical Background $\quad 17$

2.1 Maxwell's Equations $\quad 17$

$\begin{array}{lll}2.2 & \text { Constitutive Relations } & 19\end{array}$

2.3 Boundary Conditions 21

2.4 Reflection and Transmission 22

2.5 Transmission Line Theory 24

2.5.1 Transmission Lines' Equations 24

2.6 The Scattering Matrix 26

2.7 Metamaterial 27

$\begin{array}{ll}2.7 .1 & \text { Introduction } \\ 2.2 & 28\end{array}$

2.7.2 Wave Propagation in Left-Handed Media 30

2.7.3 Negative Refraction 32

2.7.4 Purely Left-Handed Transmission Line 34

2.7.5 Composite Right/Left-Handed Transmission Line 36

2.7.6 Metasurface 39

2.7.7 Characterizing a Metasurface 40

2.8 Radar Cross-Section 42

3 Electromagnetic-Wave Absorber $\quad 47$

3.1 Introduction 47

3.2 Types of Electromagnetic-Wave Absorbers 47

3.2.1 Resonant Absorbers 48

3.2.1.1 Salisbury Screen 48

3.2.1.2 Jaumann Absorber $\quad 49$

3.2.1.3 Dällenbach Layer $\quad 50$

3.2.1.4 Circuit Analog Absorber 50

3.2.2 Broadband Absorbers 51

3.2.3 Physical Mechanisms of Absorption 51

3.3 Theorems for Electromagnetic-Wave Absorber 53

3.3.1 Weston's Theorems 53

3.3.2 Rozanov's Theory 53

3.4 Metamaterial-based Absorber 54

3.5 Interpretation and Characterization of Absorbers 56

3.5.1 Equivalent Circuit $\quad 56$

3.5.2 Reflection and Transmission in a Stratified Medium 59

3.5.2.1 Reflection Coefficient 61

3.5.2.2 Reflection and Transmission in a Homogeneous Dielectric Film 62 
4 Design and Characterization of Metamaterial Absorbers $\quad 65$

$\begin{array}{lll}4.1 & \text { Parametric Approach } & 65\end{array}$

$\begin{array}{lll}\text { 4.1.1 Numerical and Conceptual Analysis } & 69\end{array}$

4.2 Mechanism of Absorption 73

4.3 Equivalent Circuit and Parameter Retrieval 80

4.4 Applications of the Proposed Metamaterial Absorber 87

4.4.1 Radar Cross-Section Reduction in Antennas $\quad 87$

4.4.1.1 RCS Analysis of a Microstrip Patch Antenna 87

4.4.1.2 RCS Analysis of a Microstrip Antenna Array 90

4.4.2 Mutual Coupling Reduction on a Spatially Close Microstrip Array 94

4.5 Experimental Results 96

$\begin{array}{lll}5 & \text { Conclusion and Future Work } & 101\end{array}$

$\begin{array}{llr}6 & \text { Bibliography } & 103\end{array}$ 


\section{List of figures}

Figure 2.1 Field directions at boundary [16].

Figure 2.2 Plane wave obliquely impinging at a dielectric interface [17].

Figure 2.3 Distributed model of a transmission line [17]. 24

Figure 2.4 Generic N-port network [17]. 27

Figure 2.5 Generic example of metamaterial [10]. 28

Figure 2.6 The system of vectors $\mathbf{E}, \mathbf{H}, \mathbf{k}$, and $\mathbf{S}$ considering a plane transverse electromagnetic (TEM) wave in a right- (a) and a left-handed (b) medium [22].

Figure 2.7 Classification of materials [10].

Figure 2.8 Prototype of the metamaterial used by Smith et al. to experimentally reproduce a DNG medium [24].

Figure 2.9 Characteristic of reflection and refraction of a impinging uniform plane wave at a DPS-DNG interface, image adapted from [15].

Figure 2.10 Photorealistic images of a drinking glass filled with water with its real index of refraction (a) and the same drinking glass with fitcious index of refraction equals to the water but negative (b) $[25]$.

Figure 2.11 Homogeneous left- (a) and right-handed (b) transmission lines.

Figure 2.12 Effectively homogeneous CRLH transmission line.

Figure 2.13 Dispersion diagram of the purely $\mathrm{RH}\left(\beta_{\mathrm{PRH}}\right)$, the purely LH $\left(\beta_{\mathrm{PLH}}\right)$ and CRLH ( $\beta_{\mathrm{RH}}$ and $\left.\beta_{\mathrm{LH}}\right)$ TLs, considering the power flow along the $+z$ direction. [26].

Figure 2.14 Free-space measurement of a material sample [33]. 42

Figure 2.15 Radar Cross Section: monostatic (a) and bistatic (b) [35]. 43

Figure 3.1 Salisbury Screen (a) and its equivalent circuit (b). 48

Figure 3.2 Jaumann absorber (a) and its equivalent circuit (b). 49

Figure 3.3 Dällenbach layer (a) and its equivalent circuit (b). $\quad 50$

Figure 3.4 Examples of FSS elements [38]. 51

Figure 3.5 Broadband absorber used in anechoic chambers [39]. 52

Figure 3.6 Unit cell of the MPA proposed by Landy (a) and the plot of absorbance (b) [9]. $\quad 56$

$\begin{array}{lll}\text { Figure 3.7 } & \text { Equivalent circuit of a resonant absorber. } & 57\end{array}$

$\begin{array}{lll}\text { Figure 3.8 } & \text { Equivalent circuit of a single-resonant absorber. } & 58\end{array}$

$\begin{array}{lll}\text { Figure } 3.9 & \text { Stratified medium. } & 60\end{array}$

Figure 3.10 Homogeneous dielectric film. 63

Figure 4.1 Unit cell of the metamaterial absorber based on square spiral: top (a) and perspective (b) views. $\quad 66$

Figure 4.2 Absorptivity of a metamaterial absorber based on square spiral. 
Figure 4.3 Simulated surface current density on the top at (a) 4.7 $\mathrm{GHz}$, (b) $10.1 \mathrm{GHz}$, and (c) $15.1 \mathrm{GHz}$, and on the bottom at (d) $4.7 \mathrm{GHz}$, (e) $10.1 \mathrm{GHz}$, and (f) $15.1 \mathrm{GHz}$.

Figure 4.4 Unit cell of the proposed metamaterial absorber: top (a) and perspective (b) views.

Figure 4.5 Absorptivity of the proposed metamaterial absorber with different dimensions as in Table 4.1.

Figure 4.6 Absorptivity of the metamaterial absorber considering both TE and TM polarization.

Figure 4.7 Absorptivity of the metamaterial absorber considering different values of incidence angle $\theta$ for TE (a) and TM (b) polarizations.

Figure 4.8 Absorptivity of the metamaterial absorber based on Model 4 considering different values of $\alpha$.

Figure 4.9 Absorptivity of the metamaterial absorber based on Model 4 considering different values of $d$.

Figure 4.10 Simulated surface current density of the proposed metamaterial absorber on the top at (a) $12.2 \mathrm{GHz}$, (b) $17.1 \mathrm{GHz}$, and (c) $19.7 \mathrm{GHz}$, and on the bottom at (d) $12.2 \mathrm{GHz}$, (e) 17.1 $\mathrm{GHz}$, and (f) $19.7 \mathrm{GHz}$.

Figure 4.11 Electric field distribution of the proposed absorber at (a) 12.2 GHz, (b) $17.1 \mathrm{GHz}$, and (c) $19.7 \mathrm{GHz}$, and the magnetic field distribution at (d) $12.2 \mathrm{GHz}$, (e) $17.1 \mathrm{GHz}$, and (f) $19.7 \mathrm{GHz} .77$

Figure 4.12 Extracted constitutive electromagnetic parameters: real (a) and imaginary (b) parts of $\epsilon_{\text {eff }}$ and $\mu_{\text {eff }}$, and the real and imaginary parts of $n_{\text {eff }}(\mathrm{c})$.

Figure 4.13 Absorptivity of the proposed absorber considering lossy and loss-free FR-4 substrates.

Figure 4.14 Absorptivity of the proposed absorber considering different substrates.

Figure 4.15 Equivalent circuit of a broadband metamaterial absorber. 81

Figure 4.16 Real and imaginary parts of the (a) admittance and (b) impedance of the proposed absorber from the EM simulation.

Figure 4.17 Curves of absorptivity of the absorber for the equivalent circuit and the EM simulation.

Figure 4.18 Final equivalent circuit for the proposed metamaterial absorber.

Figure 4.19 Curves of absorptivity for the initial and final equivalent circuit, and for the EM simulation.

Figure 4.20 Real (a) and imaginary (b) parts of the input impedance from the initial and final equivalent circuits.

Figure 4.21 Curves of absorptivity for the absorber designed to operate at the $\mathrm{X}$ band.

Figure 4.22 Top (a) and side (b) views of the microstrip antenna with the metamaterial absorbers.

Figure 4.23 Simulated reflection coefficient of the antenna with and without the absorber. 
Figure 4.24 Simulated radiation pattern of the patch antenna at 10 $\mathrm{GHz}$ of the E-plane (a) and H-plane (b).

Figure 4.25 Simulated bistatic RCS of the antenna with and without absorber at $10 \mathrm{GHz}$ at the plane yoz (a) and xoz (b). $\quad 90$

Figure 4.26 Top view of the proposed microstrip patch antenna array. 91

Figure 4.27 Simulated reflection coefficient of the proposed antenna array with and without the absorber.

Figure 4.28 Simulated radiation pattern of the microstrip antenna array at $10 \mathrm{GHz}$ of the E-plane (a) and H-plane (b).

Figure 4.29 Simulated bistatic RCS of the proposed antenna arrays with and without absorber at $10 \mathrm{GHz}$ at the plane yoz (a) and xoz (b).

Figure 4.30 Simulated $S_{21}$ of the proposed antenna arrays with and without the absorber.

Figure 4.31 Absorptivity of the proposed absorber for 5G applications. 95

Figure 4.32 Top (a) and perspective (b) views of the microstrip antenna array with the metamaterial absorber wall.

Figure 4.33 Simulated S-parameters of the array with and without the absorber wall.

Figure 4.34 Top view of the surface current density at 3.7 (a) and 3.6 (b) GHz for the arrays with and without the absorber wall, respectively.

Figure 4.35 Perspective view of the surface current density on the microstrip antenna array with the decoupling wall at $3.7 \mathrm{GHz}$.

Figure 4.36 Simulated radiation pattern of E-plane (a) and H-plane (b) at 3.6 and $3.7 \mathrm{GHz}$ for the microstrip array with and without the absorber wall, respectively.

Figure 4.37 Photograph of the fabricated metamaterial absorber proposed: top (a) and perspective (b) views.

Figure 4.38 Photograph of the setup of measurements at DLR.

Figure 4.39 Simulated and measured absorptivity of the proposed absorber under normal incidence.

Figure 4.40 Absorptivity per incidence angle plot for both (a) TE and (b) TM polarization. 


\section{List of tables}

Table 2.1 Parameters of a transmission line. 26

Table 2.2 RCS of some ordinary shapes in the optical region. 46

Table 4.1 Parameters for the models of the proposed metamaterial absorbers.

Table 4.2 Comparison of the proposed absorber with previously presented absorbers.

Table 4.3 Results of the proposed absorber considering different substrates. 


\section{List of Abreviations}

$5 \mathrm{G}$

ACS

$\mathrm{CA}$

CRLH

DGS

DNG

DPS

EBG

EM

ENG

ERR

FSS

GSTC

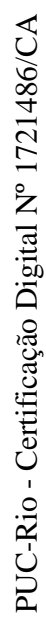

5th Generation of Mobile Technologies

Absorption Cross-Section

Circuit Analog

Composite Right/Left-Handed Transmission Line

Defected Ground Structure

Double-Negative

Double-Positive

Electromagnetic Band-Gap

Electromagnetic

Epsilon-Negative

Electric Ring Resonator

Frequency Selective Surface

Generalized Sheet Transition Conditions

Halpern Anti Radar Paint

High Frequency Structure Simulator

Left-Handed Transmission Line

Multiple-Input Multiple-Output

Metamaterial Perfect Absorber

Metamaterial

Nicolson-Ross-Weir Method

Radar Absorbing Material

Radar Cross-Section

Right-Handed Transmission Line

Transverse Electric

Transmission Line

Transverse Magnetic 
It's beauty in the struggle, ugliness in the success Hear my words or listen to my signal of distress I grew up in the city and though some times we had less

Compared to some of my homies down the block, man, we were blessed...

For what's money without happiness?

Or hard times without the people you love?

Though I'm not sure what's 'bout to happen next

I asked for strength from the Lord up above...

J. Cole, Love yourz. 


\section{Introduction}

The interest in electromagnetic-wave absorber dates back to World War II. At this time, both the United States and Germany had massive projects to develop this technology. While the United States were interested, at least at first, in the usage of absorbers to improve the radar performance by reducing the interference from the reflection of nearby objects, the main goal of Germany was in the employment of absorber in radar camouflage [1].

Over the past few decades, services using electromagnetic wave-based systems has been increasing exponentially, and absorbers are not anymore only an exclusively military issue, but civilian as well. Nowadays, absorbers are essential to a variety of applications, such as microbolometers [2], energy harvesting [3], thermal emitters [4], [5], solar cells [6], radar cross-section reduction [7], as well as the attenuation of surface waves [8]. Along with the wide applicability of the absorbers, some demanding features have been emerging over the last few years, such as compactness, broadband, polarization-independent response, and wide-angle stability. Therefore, new techniques and methods are desirable to fulfill these requirements.

In 2003, Landy et al. proposed and demonstrated a perfect absorber based on the concepts of metamaterials [9], which are engineered periodic structures that exhibit an electromagnetic response not available or not easily found in natural form [10]. The employment of metamaterials in electromagneticwave absorbers allowed an efficient way of impedance matching between the structure and the free-space, and, therefore, opening up new possibilities to the design of absorbers.

Since the perfect metamaterial absorber proposed by Landy, the interesting of the academia and industry in this kind of structure has been growing with each passing day. At the same time, many studies have been conducted to propose an equivalent circuit to describe the absorbers, as well as methods to retrieve the parameters of the circuit [11], [12], [13]. The description of absorbers as an equivalent circuit is an important tool to their understanding, analysis, and design.

Thus, in this present study, the metamaterial absorbers are extensively investigated, and their mechanism of absorption are discussed, and designed to 
operate in the microwave region. The proposed absorber is devised to present some key features, such as sub-wavelength thickness and periodicity, broadband, low-cost, polarization insensitivity, and wide-angle stability. Furthermore, an equivalent circuit and a parameter retrieval algorithm are introduced to describe broadband absorbers.

This work is organized as follows: Chapter 2 summarizes and presents necessary concepts regarding the electromagnetic theory, transmission lines, and metamaterials, which are essential to the comprehension of this work. In Chapter 3, the electromagnetic wave absorber theory is introduced and discussed, as well as methods to characterize the absorbers. Chapter 4 gathers the most important contributions of this work, and presents a design methodology to create broadband absorbers. Furthermore, an equivalent circuit and a parameter retrieval algorithm are introduced, and experimental results are detailed and discussed. The usage of the proposed absorber in microstrip antennas to reduce the radar cross-section and mutual coupling is investigated in this Chapter as well. Finally, Chapter 5 presents the conclusions, contributions, and possibilities of future works. 


\section{2}

\section{Theoretical Background}

This Chapter outlines some essential theoretical aspects to the proper understanding of the results and analysis throughout this work, including the electromagnetic, transmission line and radar cross-section theories. Furthermore, discussions on metamaterials, especially on metasurfaces, are presented.

\section{1}

\section{Maxwell's Equations}

The Maxwell's equations are governing laws that describe the behavior and the propagation of an electromagnetic (EM) wave in media and how it interacts with matter at the macroscopic level. This set of equations forms the basis of classical electromagnetism and it was established by James Clerk Maxwell in 1873 [14]. Additionally, considering that the fields are timeharmonics with a time convention of the type $e^{-i \omega t}$, Maxwell's equations in differential form can be expressed as

$$
\begin{gathered}
\nabla \times \mathbf{E}=i \omega \mathbf{B}, \\
\nabla \times \mathbf{H}=-i \omega \mathbf{D}+\mathbf{J}, \\
\nabla \cdot \mathbf{D}=\rho, \\
\nabla \cdot \mathbf{B}=0,
\end{gathered}
$$

where $\mathbf{E}$ is the electromagnetic field vector $[\mathrm{V} / \mathrm{m}], \mathbf{H}$ the magnetic field vector $[\mathrm{A} / \mathrm{m}], \mathbf{D}$ the electric flux density vector $\left[\mathrm{C} / \mathrm{m}^{2}\right]$, $\mathbf{B}$ the magnetic flux density vector $\left[\mathrm{Wb} / \mathrm{m}^{2}\right], \rho$ the charge density $\left[\mathrm{C} / \mathrm{m}^{3}\right]$, and $\mathbf{J}$ the current density vector $\left[\mathrm{A} / \mathrm{m}^{2}\right]$. Besides that, Equations 2-3 and 2-4 are the Gauss' Law for electric and magnetic fields, respectively, Equation 2-1 is the Faraday's Law of Induction, and Equation 2-2 is the Ampère's Law. Some problems are more easily solved with the integral form of the Maxwell's equations and they are deduced from the differential form using Gauss' and Stokes' theorems, and they can be written as 


$$
\begin{gathered}
\oint_{C} \mathbf{E} \cdot d \mathbf{l}=i \omega \int_{s} \mathbf{B} \cdot d \mathbf{s}, \\
\oint_{C} \mathbf{H} \cdot d \mathbf{l}=-i \omega \int_{s} \mathbf{D} \cdot d \mathbf{s}+\int_{s} \mathbf{J} \cdot d \mathbf{s}, \\
\oint_{S} \mathbf{D} \cdot d \mathbf{s}=\int_{V} \rho \cdot d v \\
\oint_{S} \mathbf{B} \cdot d \mathbf{s}=0 .
\end{gathered}
$$

The field vectors and flux density vectors are related via the so-called constitutive relations and, in free-space, they are expressed as follow

$$
\begin{aligned}
& \mathbf{D}=\varepsilon_{0} \mathbf{E}, \\
& \mathbf{B}=\mu_{0} \mathbf{H},
\end{aligned}
$$

where $\varepsilon_{0}$ and $\mu_{0}$ are the electric permittivity and the magnetic permeability of vacuum, respectively, and their values are

$$
\begin{gathered}
\varepsilon_{0}=8.854 \times 10^{-12} \simeq \frac{10^{-9}}{36 \pi}[\mathrm{F} / \mathrm{m}], \\
\mu_{0}=4 \pi \times 10^{-7}[\mathrm{H} / \mathrm{m}] .
\end{gathered}
$$

The term $\frac{\partial \mathbf{D}}{\partial t}$ is known as displacement current density and plays an important role in the Maxwell's equations. This term was added in the Ampére's Law, Equation 2-2, in order to ensure the charge conservation and it can be considered one of the most important contributions of Maxwell. So, taking the divergence of both sides of Equation 2-2 and using Gauss' Law for electric fields, the conservation law can be written as

$$
\frac{\partial \rho}{\partial t}+\nabla \cdot \mathbf{J}=0
$$

The behavior of an electromagnetic field depends on the features of the medium in which the propagation occurs. Still, no references to these features and how it affects the wave were made in this Section so far. Therefore, different types of media and their influence on the wave propagation will be approached in Section 2.2. 


\section{2}

\section{Constitutive Relations}

When materials are subjected to an EM field, their charged particles interact with the EM field vectors, producing current, and, thus, the wave propagation through the material is different when compared with the propagation in free-space [15]. To describe the interaction of EM wave with the matter, the constitutive parameters must be introduced and are given, considering a linear and isotropic medium, by

$$
\begin{aligned}
& \mathbf{D}=\varepsilon \mathbf{E}, \\
& \mathbf{B}=\mu \mathbf{H}, \\
& \mathbf{J}=\sigma \mathbf{E},
\end{aligned}
$$

where $\varepsilon$ is electric permittivity of the media $[\mathrm{F} / \mathrm{m}], \mu$ magnetic permeability $[\mathrm{H} / \mathrm{m}]$, and $\sigma$ the electrical conductivity $[\mathrm{S} / \mathrm{m}]$. Besides that, the electric permittivity $\varepsilon$ and magnetic permeability $\mu$ indicate how well the material stores electric and magnetic energy, respectively, and their general form can be written as

$$
\begin{gathered}
\varepsilon=\varepsilon^{\prime}-j \varepsilon^{\prime \prime}=\varepsilon_{0}(1+\chi), \\
\mu=\mu^{\prime}-j \mu^{\prime \prime}=\mu_{0}\left(1+\chi_{m}\right),
\end{gathered}
$$

where $\chi$ and $\chi_{m}$ are the electric and magnetic susceptibilities, respectively. The electric susceptibility is the measure of how easily bound charges are displaced due to an applied electric field. On the other hand, the magnetic susceptibility is the measure of how easily magnetic dipoles are aligned due to an applied magnetic field.

Considering a dielectric material, an applied electric field $\mathbf{E}$ implies in a displacement of electron cloud, creating electric dipole moments and polarizing the material. The influence of this effect is incorporated into the electric flux density vector $\mathbf{D}$, which can be expressed, considering the Equation 2-17, as

$$
\mathbf{D}=\varepsilon \mathbf{E}=\varepsilon_{0}(1+\chi) \mathbf{E}=\varepsilon_{0} \mathbf{E}+\mathbf{P},
$$

where $\mathbf{P}=\varepsilon_{0} \chi \mathbf{E}$ is the electric polarization and is considered the material response due to an applied electric field, while the term $\varepsilon_{0} \mathbf{E}$ is the free-space response.

The same reasoning can be used considering a magnetic material, and the magnetic flux density vector $\mathbf{B}$ can be expressed as 


$$
\mathbf{B}=\mu \mathbf{H}=\mu_{0}\left(1+\chi_{m}\right) \mathbf{H}=\mu_{0} \mathbf{H}+\mathbf{M},
$$

where $\mathbf{M}=\mu_{0} \chi_{m} \mathbf{H}$ is the magnetization and is considered the material response due to an applied magnetic field, while the term $\mu_{0} \mathbf{H}$ is the freespace response. Furthermore, one can realize that depending on the nature of the matter, contributions in the fluxes $\mathbf{D}$ and $\mathbf{B}$ arise from its polarization. These contributions are taken into account adding electric polarization $\mathbf{P}$ and magnetization $\mathbf{M}$ to the constitutive relations.

In order to make the understanding of the constitutive parameters easier, they are often referred to as relative permittivity $\varepsilon_{r}$ and relative permeability $\mu_{r}$, and are expressed as

$$
\begin{gathered}
\varepsilon_{r}=\frac{\varepsilon}{\varepsilon_{0}}=1+\chi, \\
\mu_{r}=\frac{\mu}{\mu_{0}}=1+\chi_{m} .
\end{gathered}
$$

Some important parameters of the media are expressed in terms of the permittivity and permeability, such as the characteristic impedance, refractive index, and wave number that are defined, respectively, by

$$
\begin{gathered}
\eta=\sqrt{\frac{\mu}{\varepsilon}}, \\
n=\sqrt{\varepsilon_{r} \mu_{r}}, \\
k=k_{0} \sqrt{\varepsilon \mu},
\end{gathered}
$$

where $k_{0}=$ is the free-space wave number.

When both $\mathbf{E}$ and $\mathbf{P}$ present the same direction, the material is called isotropic. Otherwise, if this feature is not present, the material is called anisotropic, and, thus, becoming the constitutive relations more complicated. In anisotropic materials, the permittivity and permeability are interpreted as tensors of rank two, and $\mathbf{D}$ and $\mathbf{B}$ can be written as

$$
\begin{gathered}
\mathbf{D}=\left[\begin{array}{l}
D_{x} \\
D_{y} \\
D_{z}
\end{array}\right]=\left[\begin{array}{lll}
\varepsilon_{x x} & \varepsilon_{x y} & \varepsilon_{x z} \\
\varepsilon_{y x} & \varepsilon_{y y} & \varepsilon_{y z} \\
\varepsilon_{z x} & \varepsilon_{z y} & \varepsilon_{z z}
\end{array}\right]\left[\begin{array}{l}
E_{x} \\
E_{y} \\
E_{z}
\end{array}\right]=[\varepsilon]\left[\begin{array}{c}
E_{x} \\
E_{y} \\
E_{z}
\end{array}\right], \\
\mathbf{B}=\left[\begin{array}{l}
B_{x} \\
B_{y} \\
B_{z}
\end{array}\right]=\left[\begin{array}{lll}
\mu_{x x} & \mu_{x y} & \mu_{x z} \\
\mu_{y x} & \mu_{y y} & \mu_{y z} \\
\mu_{z x} & \mu_{z y} & \mu_{z z}
\end{array}\right]\left[\begin{array}{l}
B_{x} \\
B_{y} \\
B_{z}
\end{array}\right]=[\mu]\left[\begin{array}{l}
B_{x} \\
B_{y} \\
B_{z}
\end{array}\right] .
\end{gathered}
$$


When $\varepsilon$ and/or $\mu$ depend on the magnitude $E$ or $H$ of applied electric or magnetic field, respectively, the medium is called non-linear, and $\mathbf{D}$ and $\mathbf{B}$ are expressed as

$$
\begin{aligned}
\mathbf{D} & =\varepsilon(E) \mathbf{E}, \\
\mathbf{B} & =\mu(H) \mathbf{H} .
\end{aligned}
$$

When $\varepsilon$ and/or $\mu$ vary depending on the location within the material, this medium is called inhomogeneous, which provides the following relation

$$
\begin{aligned}
& \mathbf{D}=\varepsilon(\mathbf{r}) \mathbf{E}, \\
& \mathbf{B}=\mu(\mathbf{r}) \mathbf{H} .
\end{aligned}
$$

\section{3}

\section{Boundary Conditions}

In practice, there is no infinite medium, which means that a propagating electromagnetic wave will eventually impinge on an interface between two media. The boundary conditions are applied to relate the fields across the interface and they can be deduced from the integral form of Maxwell's equations. Moreover, the boundary conditions for the field vectors, as it is illustrated in Figure 2.1, across the interface are given as

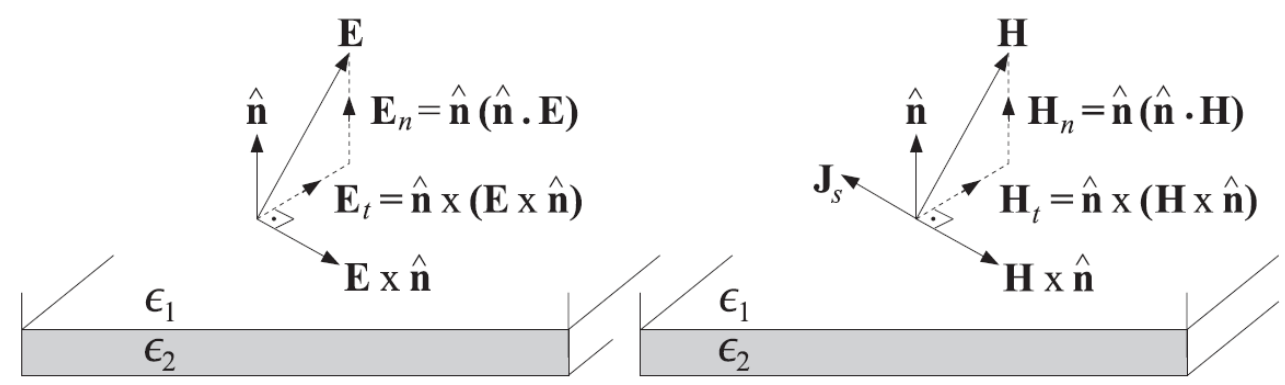

Figure 2.1: Field directions at boundary [16].

$$
\begin{gathered}
\hat{\mathbf{n}} \times\left(\mathbf{E}_{1}-\mathbf{E}_{2}\right)=0, \\
\hat{\mathbf{n}} \times\left(\mathbf{H}_{1}-\mathbf{H}_{2}\right)=\mathbf{J}_{s}, \\
\hat{\mathbf{n}} \cdot\left(\mathbf{B}_{1}-\mathbf{B}_{2}\right)=0, \\
\hat{\mathbf{n}} \cdot\left(\mathbf{D}_{1}-\mathbf{D}_{2}\right)=\rho_{s},
\end{gathered}
$$

where the subscripts 1 and 2 indicate the field vectors of the medium 1 and 2, respectively, $\hat{\mathbf{n}}$ is a unit vector normal to the interface pointing from medium 
2 into medium 1, and $\mathbf{J}_{s}$ and $\rho_{s}$ are the electric surface current and surface charge density across the interface, respectively [16].

\section{4}

\section{Reflection and Transmission}

When an incident plane wave hits a media interface, part of the energy is reflected and part is transmitted. Considering the situation in Figure 2.2, where the incident wave propagates into the interface in the $+z$ direction, and the incidence is oblique with parallel polarization in a dielectric interface, and, therefore, for $z<0$, the incident electric and magnetic fields can be written as

$$
\begin{gathered}
\vec{E}_{i}=E_{0}\left(\hat{x} \cos \theta_{i}-\hat{z} \sin \theta_{i}\right) e^{-j k_{1}\left(x \sin \theta_{i}+z \cos \theta_{i}\right)}, \\
\vec{H}_{i}=\frac{E_{0}}{\eta_{1}} \hat{y} e^{-j k_{1}\left(x \sin \theta_{i}+z \cos \theta_{i}\right)}
\end{gathered}
$$

where $k_{1}$ and $\eta_{1}$ are the propagation constant and the characteristic impedance of the region I, respectively, and $E_{0}$ is the amplitude of the incident wave. Moreover, the reflected wave can be expressed as

$$
\begin{gathered}
\vec{E}_{r}=E_{0} \Gamma\left(\hat{x} \cos \theta_{r}+\hat{z} \sin \theta_{r}\right) e^{-j k_{1}\left(x \sin \theta_{r}-z \cos \theta_{r}\right)}, \\
\vec{H}_{r}=\frac{-E_{0} \Gamma}{\eta_{1}} \hat{y} e^{-j k_{1}\left(x \sin \theta_{r}-z \cos \theta_{r}\right)},
\end{gathered}
$$

where $\Gamma$ is the reflection coefficient. For $z>0$, the transmitted electric and magnetic fields are represented as follow

$$
\begin{gathered}
\vec{E}_{t}=E_{0} \mathrm{~T}\left(\hat{x} \cos \theta_{t}-\hat{z} \sin \theta_{t}\right) e^{-j k_{2}\left(x \sin \theta_{t}+z \cos \theta_{t}\right)}, \\
\vec{H}_{t}=\frac{E_{0} \mathrm{~T}}{\eta_{2}} \hat{y} e^{-j k_{2}\left(x \sin \theta_{t}+z \cos \theta_{t}\right)}
\end{gathered}
$$

where $k_{2}$ and $\eta_{2}$ are the propagation constant and the characteristic impedance of the region II, respectively, and $\mathrm{T}$ is the transmission coefficient. As it is stated in Section 2.3, the tangential components of the electric and magnetic fields are continuous at the media interface $(z=0)$, so

$$
\begin{aligned}
\cos \theta_{i} e^{-j k_{1} x \sin \theta_{i}}+\Gamma \cos \theta_{r} e^{-j k_{1} x \sin \theta_{r}} & =\mathrm{T} \cos \theta_{t} e^{-j k_{2} x \sin \theta_{t}}, \\
\frac{1}{\eta_{1}} e^{-j k_{1} x \sin \theta_{i}}-\frac{\Gamma}{\eta_{1}} e^{-j k_{1} x \sin \theta_{r}} & =\frac{\mathrm{T}}{\eta_{2}} e^{-j k_{2} x \sin \theta_{t}}
\end{aligned}
$$

In order to solve these two equations above, the following phase match condition must be satisfied

$$
k_{1} \sin \theta_{i}=k_{1} \sin \theta_{r}=k_{2} \sin \theta_{t} .
$$

Once the phase match condition is respected, the reflection and transmission coefficients for oblique incidence can be defined as 


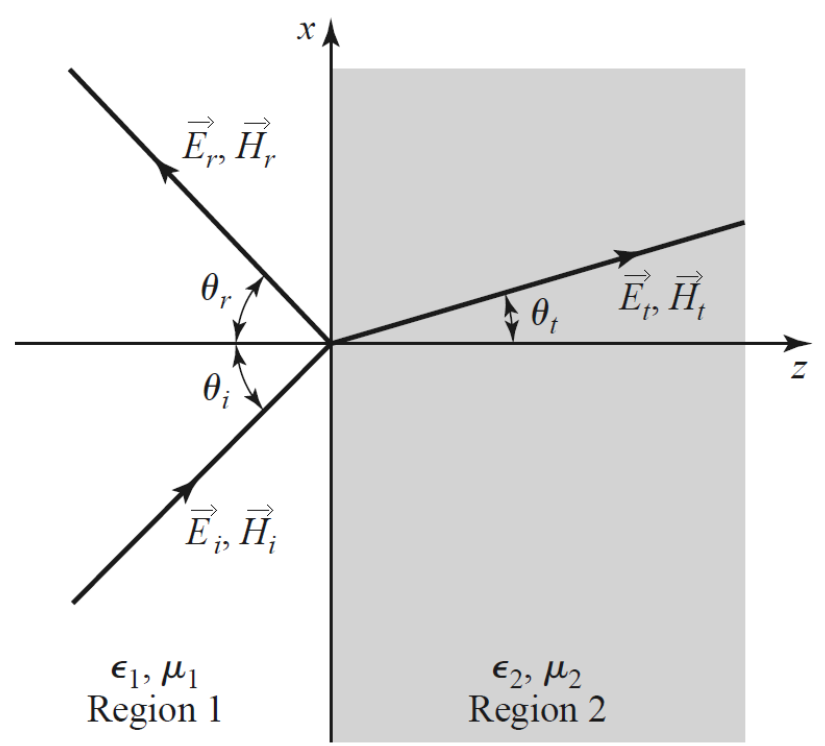

Figure 2.2: Plane wave obliquely impinging at a dielectric interface [17].

$$
\begin{aligned}
\Gamma & =\frac{\eta_{2} \cos \theta_{t}-\eta_{1} \cos \theta_{i}}{\eta_{2} \cos \theta_{t}+\eta_{1} \cos \theta_{i}}, \\
\mathrm{~T} & =\frac{2 \eta_{2} \cos \theta_{i}}{\eta_{2} \cos \theta_{t}+\eta_{1} \cos \theta_{i}} .
\end{aligned}
$$

Under normal incidence, where $\theta_{i}=\theta_{r}=\theta_{t}=0$, the reflection and transmission coefficients are

$$
\begin{aligned}
\Gamma & =\frac{\eta_{2}-\eta_{1}}{\eta_{2}+\eta_{1}}, \\
\mathrm{~T} & =\frac{2 \eta_{2}}{\eta_{2}+\eta_{1}} .
\end{aligned}
$$

So far, the development to find the reflection and transmission coefficients in this Section considered the parallel polarization. The same reasoning can be applied for the perpendicular polarization, but taking into account the proper magnetic and electric fields for the type of polarization at issue [17]. Therefore, the reflection and transmission coefficients for the perpendicular polarization are written as

$$
\begin{aligned}
\Gamma & =\frac{\eta_{2} \cos \theta_{i}-\eta_{1} \cos \theta_{t}}{\eta_{2} \cos \theta_{i}+\eta_{1} \cos \theta_{t}}, \\
\mathrm{~T} & =\frac{2 \eta_{2} \cos \theta_{i}}{\eta_{2} \cos \theta_{i}+\eta_{1} \cos \theta_{t}} .
\end{aligned}
$$

It is worth pointing out that the expressions for the reflection and transmission coefficients under normal incidence are the same for both perpendicular and parallel polarization. 


\section{5}

\section{Transmission Line Theory}

Transmission lines (TL) are used to carry electrical signals from one point to another, and, thus, it is easy to realize that TLs are extensively used in everyday applications. Additionally, the main examples of them are the coaxial cable, microstrip line, and twisted pair cable. Basically, a TL consists of at least two electrical conductors that are used to carry the signal.

Lumped element circuit concepts play an important role in TL theory and are frequently used. Despite that, TLs cannot be analyzed or described by circuit theory. The main difference between the TL and circuit theories relies on their electrical size. In TL theory, the physical size of the line is much smaller than the guided wavelength $\left(\lambda_{g}\right)$ or even a fraction, while in circuit theory the physical size of the network is smaller than the wavelength, but not so smaller when comparing with TL theory. So, TL is considered a distributed parameter network, i.e. their parameters are defined by unit per length.

Figure 2.3 shows a distributed model representing a two-wire transmission line of infinitesimal length $\Delta z$, where $L$ is the series inductance in $[\mathrm{H} / \mathrm{m}]$, $R$ the series resistance in $[\Omega / \mathrm{m}], C$ the shunt capacitance $[\mathrm{F} / \mathrm{m}]$ and $G$ the shunt conductance $[\mathrm{S} / \mathrm{m}]$.

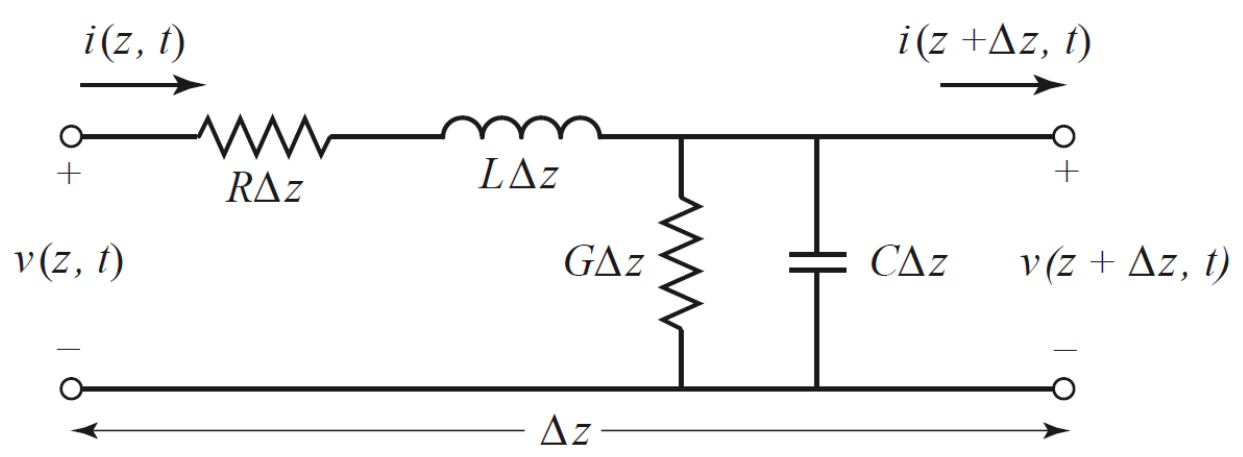

Figure 2.3: Distributed model of a transmission line [17].

\subsection{1}

\section{Transmission Lines' Equations}

Applying Kirchhoff's Voltage Law to the outer loop of the equivalent circuit of Figure 2.3 is possible to find

$$
\begin{gathered}
v(z, t)=R \Delta z i(z, t)+L \Delta z \frac{\partial i(z, t)}{\partial t}+v(z+\Delta z, t), \\
-\frac{v(z+\Delta z, t)-v(z, t)}{\Delta z}=R i(z, t)+L \frac{\partial i(z, t)}{\partial t} .
\end{gathered}
$$


Taking the limit of Equation 2-52 as $\Delta z \longrightarrow 0$, the following equation is found

$$
-\frac{\partial v(z, t)}{\partial z}=\operatorname{Ri}(z, t)+L \frac{\partial i(z, t)}{\partial t} .
$$

Now, applying Kirchhoff's current law to the main node of Figure 2.3, it is possible to obtain

$$
\begin{gathered}
i(z, t)=G \Delta z v(z+\Delta z, t)+C \Delta z \frac{\partial v(z+\Delta z, t)}{\partial t}+i(z+\Delta z, t), \\
-\frac{i(z+\Delta z, t)-i(z, t)}{\Delta z}=G v(z+\Delta z, t)+C \frac{\partial v(z+\Delta z, t)}{\partial t} .
\end{gathered}
$$

Taking the limit of Eq. 2-55 as $\Delta z \longrightarrow 0$, the following equation is found

$$
-\frac{\partial i(z, t)}{\partial z}=G v(z, t)+C \frac{\partial v(z, t)}{\partial t} .
$$

So, Equations 2-53 and 2-56 are the time domain form of TL's equation and describe the behavior of current and tension over any transmission line.

Considering a time-harmonic dependence, Equations 2-53 and 2-56 can be rewritten as

$$
\begin{aligned}
& -\frac{d V(z)}{d z}=(R+j \omega L) I(z), \\
& -\frac{d I(z)}{d z}=(G+j \omega C) V(z),
\end{aligned}
$$

where $V(z)$ and $I(z)$ are the phasorial forms of $v(z, t)$ and $i(z, t)$, respectively.

In Equations 2-57 and 2-58, $V(z)$ and $I(z)$ are coupled. Therefore, taking the derivative in terms of these equations, it is possible to find the following expressions

$$
\begin{aligned}
& \frac{d^{2} V(z)}{d z^{2}}=(R+j \omega L)(G+j \omega C) V(z), \\
& \frac{d^{2} I(z)}{d z^{2}}=(G+j \omega C)(R+j \omega L) I(z) .
\end{aligned}
$$

Rearranging these equations

where

$$
\begin{aligned}
& \frac{d^{2} V(z)}{d z^{2}}-\gamma^{2} V(z)=0 \\
& \frac{d^{2} I(z)}{d z^{2}}-\gamma^{2} I(z)=0
\end{aligned}
$$

$$
\gamma=\alpha+j \beta=\sqrt{(R+j \omega L)(G+j \omega C)} .
$$

It is easy to realize that Equations 2-61 and 2-62 are the wave equations of tension and current in a transmission line. Besides that, $\gamma$ is known as the 
complex propagation constant, $\alpha$ the attenuation constant and $\beta$ the phase constant. The traveling wave solution of the wave equations can be expressed as

$$
\begin{gathered}
V(z)=V_{0}^{+} e^{-\gamma z}+V_{0}^{-} e^{+\gamma z}, \\
I(z)=I_{0}^{+} e^{-\gamma z}+I_{0}^{-} e^{+\gamma z} .
\end{gathered}
$$

where $V_{0}^{+}, V_{0}^{-}, I_{0}^{+}$and $I_{0}^{-}$are the amplitudes of the wave. Moreover, $e^{-\gamma z}$ and $e^{+\gamma z}$ indicate a propagation in $+\mathrm{z}$ and $-\mathrm{z}$ directions, respectively. Applying Equation 2-57 in Equations 2-64 and 2-65, it is possible to find the following relation

$$
I_{0}^{+} e^{-\gamma z}+I_{0}^{-} e^{+\gamma z}=\frac{\gamma}{R+j \omega L}\left(V_{0}^{+} e^{-\gamma z}-V_{0}^{-} e^{+\gamma z}\right) .
$$

The characteristic impedance of the transmission line, $Z_{0}$, can be defined as

$$
Z_{0}=\frac{R+j \omega L}{\gamma}=\sqrt{\frac{R+j \omega L}{G+j \omega C}} .
$$

In addition, the characteristic impedance can be defined as the ratio between the tension wave and current wave that propagate in the same direction. Thus,

$$
Z_{0}=\frac{V_{0}^{+}}{I_{0}^{+}}=-\frac{V_{0}^{-}}{I_{0}^{-}}
$$

So far, the analysis considered a transmission line with losses. However, in some cases, a lossless line will be considered, where $R=0=G$. Another important situation is the distortionless line, where $\frac{R}{L}=\frac{G}{C}$. For these reasons, Table 2.1 sums up the relations for $\gamma$ and $Z_{0}$ considering a transmission line with loss, a lossless line, and a distortionless line.

Table 2.1: Parameters of a transmission line.

\begin{tabular}{c|c|c} 
Case & $\gamma$ & $Z_{0}$ \\
\hline General & $\sqrt{(R+j \omega L)(G+j \omega C)}$ & $\sqrt{\frac{R+j \omega L}{G+j \omega C}}$ \\
Lossless & $0+j \omega \sqrt{L C}$ & $\sqrt{\frac{L}{C}}+j 0$ \\
Distortionless & $\sqrt{R G}(1+j \omega \sqrt{L C})$ & $\sqrt{\frac{L}{C}}+j 0$
\end{tabular}

\section{6}

\section{The Scattering Matrix}

The scattering matrix, or S-matrix for short, is an important tool to the understanding of the behavior of an N-port network and it provides a relation 
between the incident and reflected voltage waves. Figure 2.4 shows a generic $\mathrm{N}$-port network and its S-matrix is represented as

$$
\left[\begin{array}{c}
V_{1}^{-} \\
V_{2}^{-} \\
\vdots \\
V_{\mathrm{N}}^{-}
\end{array}\right]=\left[\begin{array}{cccc}
S_{11} & S_{12} & \ldots & S_{1 \mathrm{~N}} \\
S_{21} & \ddots & & \vdots \\
\vdots & & \ddots & \vdots \\
S_{\mathrm{N} 1} & \ldots & \ldots & S_{\mathrm{NN}}
\end{array}\right]\left[\begin{array}{c}
V_{1}^{+} \\
V_{2}^{+} \\
\vdots \\
V_{\mathrm{N}}^{+}
\end{array}\right]
$$

where $V_{\mathrm{N}}^{-}$and $V_{\mathrm{N}}^{+}$are the amplitude of the reflected and incident voltage waves of the port $n$, respectively. Additionally, the S-matrix can be represented in a simple form as

$$
\left[V^{-}\right]=[S]\left[V^{+}\right]
$$

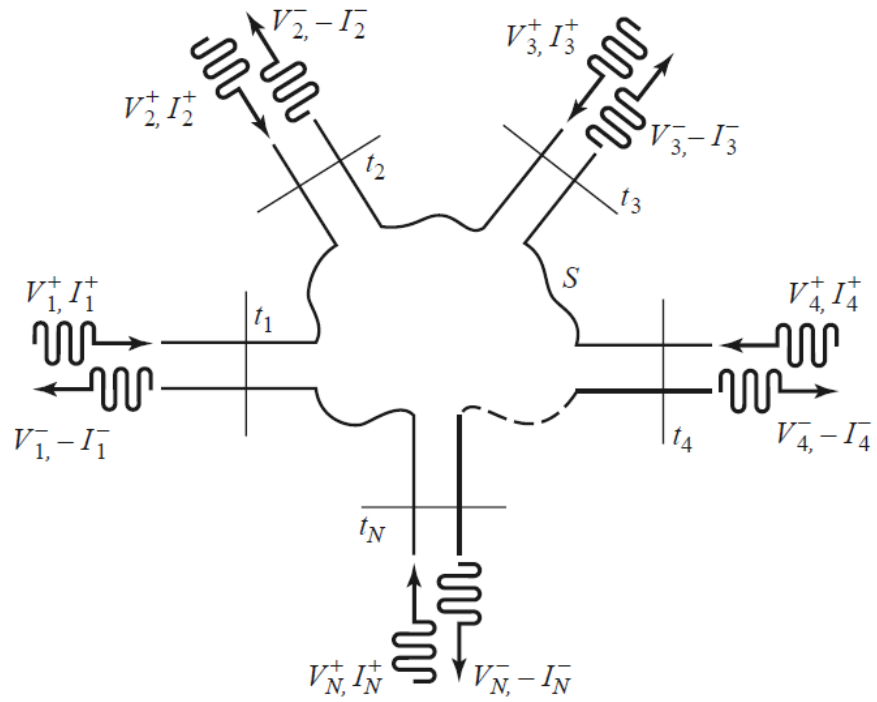

Figure 2.4: Generic N-port network [17].

The individual elements of the S-matrix are calculated setting to zero the incident wave of all ports but the jth port and it can be expressed as follows

$$
S_{\mathrm{ij}}=\left.\frac{V_{\mathrm{i}}^{-}}{V_{\mathrm{j}}^{+}}\right|_{V_{\mathrm{k}}^{+}=0 \forall k \neq i} .
$$




\subsection{1}

\section{Introduction}

Electromagnetic metamaterials, or simply metamaterials (MTM), are engineered periodic structures that exhibit unique and unusual electromagnetic properties, which are not ordinarily found in nature. The lattice constants of a single unit cell of MTM must be much smaller than the free-space and/or guided wavelength so that a macroscopic electromagnetic response can be considered and tailored. In general, an MTM can be synthesized by a subwavelength periodic array of resonators embedded or etched in a host medium or surface, as it can be seen in Figure 2.5. Besides that, these features allow the MTMs to find a great number of applications as in antenna systems [18], absorbers [9], polarizers [19], and perfect lens [20], to name a few.
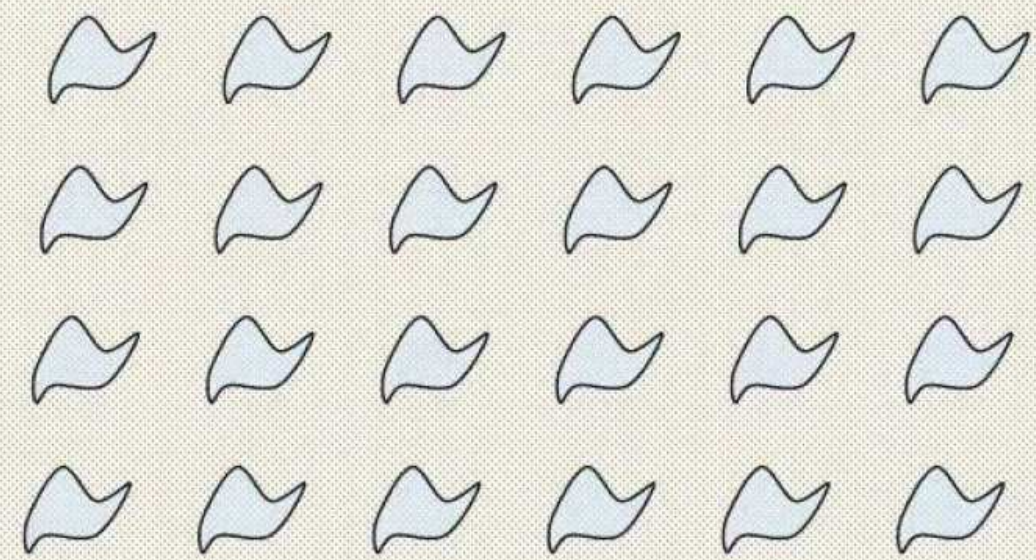

Figure 2.5: Generic example of metamaterial [10].

Using the effective medium theory, MTMs can be characterized and classified in terms of their complex permittivity and permeability. In addition, the types of MTMs that receive most of the attention from academia are known as double-negative media materials, which are composite media with their effective permittivity and permeability are negative real values simultaneously and are often called left-handed media. This possibility was theoretically investigated by V.G. Veselago in 1967 [21], whose work anticipated the phenomena that occur when a monochromatic uniform plane wave travels through a left-handed medium. He realized the occurrence of exotic electromagnetic features such as the reversal of Snell's Law, the Doppler effect, and the Vavilov-Cerenkov effect [21]. Moreover, he postulated that a propagating wave in such medium presents the Poyting vector direction that is antiparallel to the direction of 
the phase velocity, and, thus, in this kind of medium, the triplet $\mathbf{E}, \mathbf{H}$ and $\mathbf{k}$ does not follow the right-hand rule, it follows the left-hand rule. Figure 2.6 provides the illustration of the system of vector $\mathbf{E}, \mathbf{H}, \mathbf{k}$, and $\mathbf{S}$ for both rightand left-handed media.

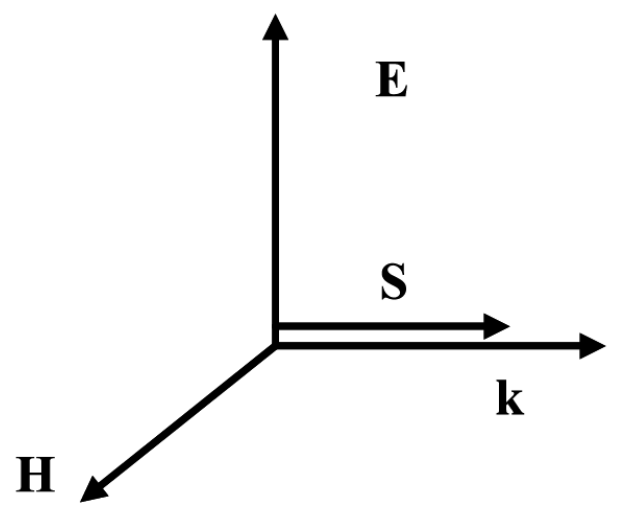

(a)

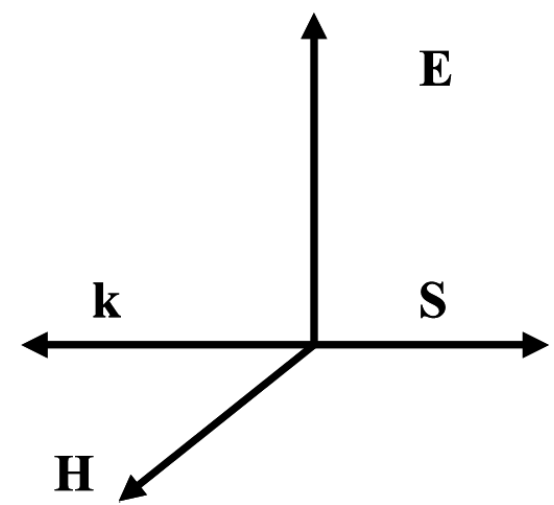

(b)

Figure 2.6: The system of vectors $\mathbf{E}, \mathbf{H}, \mathbf{k}$, and $\mathbf{S}$ considering a plane transverse electromagnetic (TEM) wave in a right- (a) and a left-handed (b) medium [22].

In general, the materials can be classified in terms of their macroscopic permeability and permittivity, as can be observed in Figure 2.7. The materials with both positive permittivity and permeability $(\epsilon>0, \mu>0)$ are known as double-positive (DPS) media/materials. The dielectrics are the most common example of DPS media. In the second quadrant falls the media with negative permittivity and positive permeability $(\epsilon<0, \mu>0)$ that are known as epsilon-negative (ENG). Noble metals in the infrared and visible frequency ranges and plasmas are examples of ENG. A mu-negative (MNG) medium presents positive permittivity and negative permeability $(\epsilon>0, \mu<0)$ and magnetic materials are examples of it. A medium with both permittivity and permeability with negative values $(\epsilon<0, \mu<0)$ is referred as double-negative (DNG). When it comes to metamaterials, DNGs are the most explored class of material and they are physically realizable only with artificial structures.

In 1999, Pendry et al. published a paper theoretically proving that an array of split-ring resonators and metal wires could provide negative permeability and permittivity, and, therefore, achieving a negative index of refraction [23]. The first experimental verification of DNG media was carried out by Smith et al. in 2001 [24] and Figure 2.8 shows the structure used in this work. Moreover, in this structure, the split-ring resonator contributes to the negative permeability and the metal wire contributes to the negative permittivity, and both together, arranged periodically, create a DNG medium. Since these breakthroughs, the expression metamaterial has become a buzzword in 


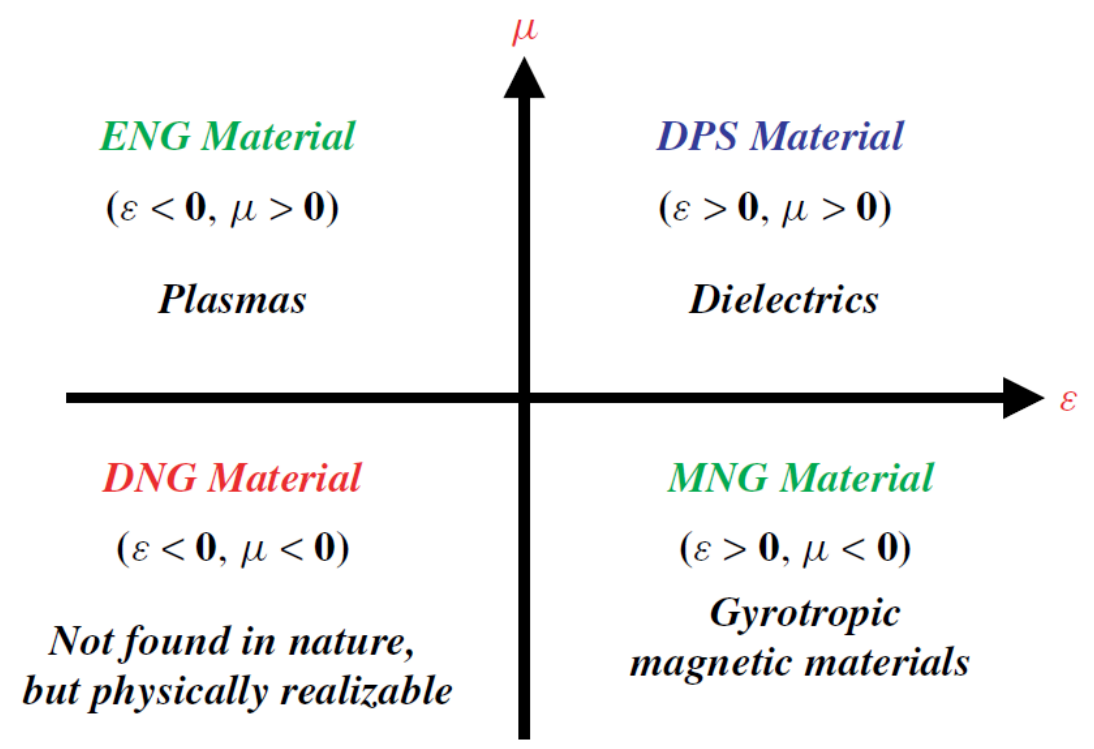

Figure 2.7: Classification of materials [10].

academia and industry due to its great range of new possibilities in both electromagnetic and material fields.

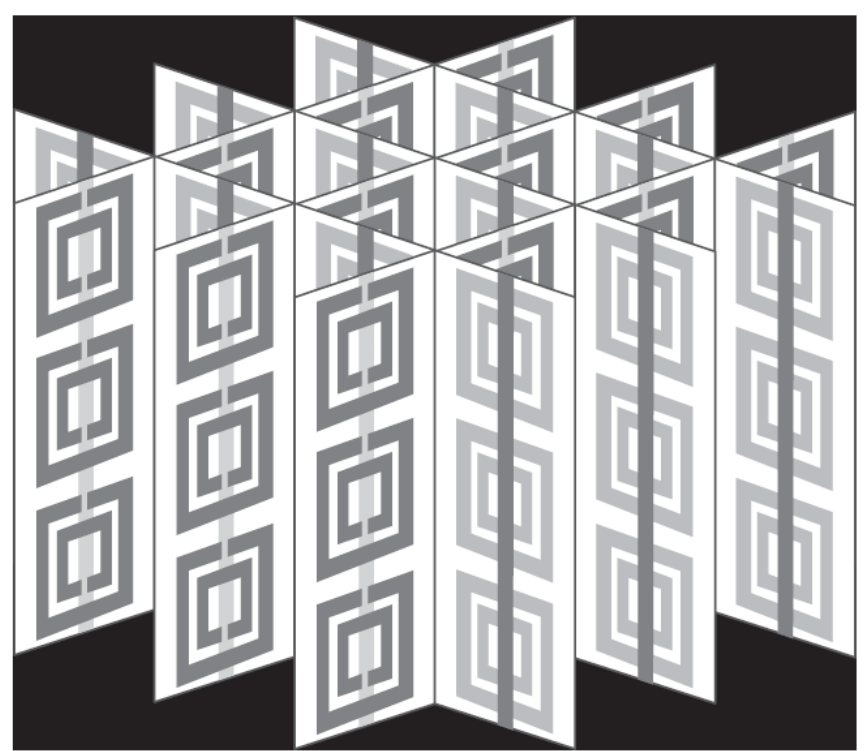

Figure 2.8: Prototype of the metamaterial used by Smith et al. to experimentally reproduce a DNG medium [24].

\subsection{2}

\section{Wave Propagation in Left-Handed Media}

To understand the propagation in a left-handed medium, it is necessary to consider that the Maxwell's equations are reduced, considering an electro- 
magnetic wave in a medium with permittivity $\epsilon$ and permeability $\mu$, to the following wave equations [22],

$$
\begin{aligned}
\nabla^{2} \mathbf{E}+\omega^{2} \mu \epsilon \mathbf{E} & =0, \\
\nabla^{2} \mathbf{H}+\omega^{2} \mu \epsilon \mathbf{H} & =0,
\end{aligned}
$$

from which the plane wave fields are expressed as follow

$$
\begin{aligned}
& \mathbf{E}=E_{0} e^{-j(\mathbf{k} \cdot \mathbf{r}-\omega t)}, \\
& \mathbf{H}=H_{0} e^{-j(\mathbf{k} \cdot \mathbf{r}-\omega t)},
\end{aligned}
$$

where the time and space dependence of the fields are of the form $e^{-j(\mathbf{k} \cdot \mathbf{r}-\omega t)}$. It is clear that the considered plane wave can propagate in a medium where the product between $\epsilon$ and $\mu$ is positive, which means that the propagation in left-handed media is possible.

Once the possibility of propagation of the electromagnetic wave in a left-handed media is confirmed, it is time to verify the difference between the propagation in DPS and DNG media. So, let's consider the following Maxwell's first order equations

$$
\begin{gathered}
\nabla \times \mathbf{E}=-j \omega \mu \mathbf{H}, \\
\nabla \times \mathbf{H}=j \omega \epsilon \mathbf{E} .
\end{gathered}
$$

Solving these equations considering the plane wave at issue in a DPS medium, it is possible to find

$$
\begin{aligned}
\mathbf{k} \times \mathbf{E} & =\omega \mu \mathbf{H}, \\
\mathbf{k} \times \mathbf{H} & =-\omega \epsilon \mathbf{E} .
\end{aligned}
$$

So, it can be inferred that the triplet of vectors $\mathbf{E}, \mathbf{H}$, and $\mathbf{k}$ forms the righthanded rule, which is expected for a medium where both $\epsilon$ and $\mu$ are positive. On the other hand, for a DNG medium,

$$
\begin{gathered}
\mathbf{k} \times \mathbf{E}=-\omega|\mu| \mathbf{H}, \\
\mathbf{k} \times \mathbf{H}=\omega|\epsilon| \mathbf{E} .
\end{gathered}
$$

These relations show that, for a medium where both $\epsilon$ and $\mu$ are negative, the triplet of vectors $\mathbf{E}, \mathbf{H}$, and $\mathbf{k}$ forms the left-handed orthogonal system. 
The direction of propagation of an electromagnetic wave is provided by the orientation of the Poynting vector, which is given by

$$
\mathbf{S}=\frac{1}{2} \operatorname{Re}\left[\mathbf{E} \times \mathbf{H}^{*}\right]
$$

It can be inferred from the Poynting vector that its direction does not depend on whether the medium is DPS or DNG, and the triplet of vector $\mathbf{E}, \mathbf{H}$ and $\mathbf{S}$ forms the right-handed rule in both media. Hence, in a DNG medium, the direction of the Poynting vector $\mathbf{S}$ is antiparallel to the direction of the wave vector $\mathbf{k}$, which means that the direction of propagation is opposite to the direction of the phase velocity. This phenomenon is illustrated in Figure 2.6.

\subsection{3}

\section{Negative Refraction}

As it was aforementioned, a DNG medium possesses negative permittivity and permeability at the same time, and, thus, this medium has a negative index of refraction. This kind of medium is not found in natural form and presents some different features from the conventional DPS media. Moreover, the index of refraction of a medium is frequency-dependent and can be written as

$$
n(\omega)^{2}=\epsilon(\omega) \mu(\omega) .
$$

The index of refraction provides the measure of the deflection in the propagation of an electromagnetic wave when it hits an interface between two media with different values of refractive indices. Besides that, the Snell's Law of refraction provides a relation among the angle of incidence, angle of refraction, and indices of refraction of these two media, and it is represented as follows

$$
n_{1} \sin \theta_{1}=n_{2} \sin \theta_{2} .
$$

where $n_{1}$ and $n_{2}$ are the indices of refraction of the media 1 and 2, respectively, and $\theta_{1}$ and $\theta_{2}$ are the angle of the ray tracing of the propagating wave with the surface normal of media 1 and 2, respectively. Besides that, at an interface, the Snell's Law of reflection must be satisfied, which is written as

$$
\theta_{r}=\theta_{i}
$$

where $\theta_{r}$ and $\theta_{i}$ are the angle of reflection and incidence, respectively.

Considering that medium 1 is DPS and medium 2 is DNG, and, thus, $n_{1}>0$ and $n_{2}<0$, the interface between these two media presents different properties of refraction from a conventional DPS-DPS interface, as can be seen, for a perpendicular polarization, in Figure 2.9. In this Figure, $\mathbf{S}$ denotes the Poynting vectors and $\mathbf{k}$, the phase vectors. Besides that, at a DPS-DPS interface, the refracted wave presents the same direction of propagation of the 
incident wave. On the other hand, at a DPS-DNG interface, the direction of propagation of the refracted wave is opposite to the direction of the incident wave and, as aforementioned in Subsection 2.7.2, the direction of the power flow is antiparallel to the phase vector. In a DNG medium, the phase wavefronts move toward the source, which can be considered unusual when comparing to a DPS medium, where the phase wavefronts move outward from the source. This phenomenon can be inferred from the expression of the propagation constant for the DNG medium, that is represented as follows

$$
k_{2}=\omega \sqrt{\epsilon_{2} \mu_{2}}=-\omega \sqrt{\left|\epsilon_{2}\right|\left|\mu_{2}\right|},
$$

where the minus sign indicates a phase advance, which is a key characteristic of a DNG medium. In a DPS medium, the propagation constant $k_{1}=\omega \sqrt{\epsilon_{1} \mu_{1}}$ provides a phase delay and this is one of the main differences between the media at issue.

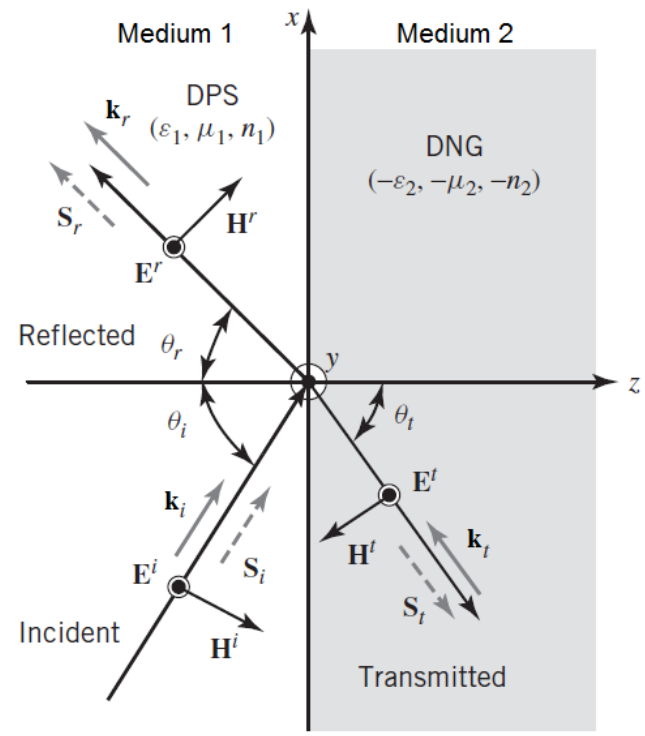

Figure 2.9: Characteristic of reflection and refraction of a impinging uniform plane wave at a DPS-DNG interface, image adapted from [15].

The antiparallelism between the power flow and the phase vector can be better understood if these vectorial quantities were described mathematically. So, considering that the phase match conditions between $k_{1}$ and $k_{2}$ are respected at the interface, and, thus, considering the Figure 2.9, the wave vectors for the incident, reflected and transmitted fields can be represented as

$$
\begin{aligned}
& \mathbf{k}_{i}=k_{1}\left(\hat{x} \cdot \sin \theta_{i}+\hat{z} \cdot \cos \theta_{i}\right)=n_{1} \frac{\omega}{c}\left(\hat{x} \cdot \sin \theta_{i}+\hat{z} \cdot \cos \theta_{i}\right), \\
& \mathbf{k}_{r}=k_{1}\left(\hat{x} \cdot \sin \theta_{i}-\hat{z} \cdot \cos \theta_{i}\right)=n_{1} \frac{\omega}{c}\left(\hat{x} \cdot \sin \theta_{i}-\hat{z} \cdot \cos \theta_{i}\right),
\end{aligned}
$$




$$
\mathbf{k}_{t}=\left|k_{2}\right|\left(\hat{x} \cdot \sin \left|\theta_{t}\right|-\hat{z} \cdot \cos \left|\theta_{t}\right|\right)=\left|n_{2}\right| \frac{\omega}{c}\left(\hat{x} \cdot \sin \left|\theta_{t}\right|-\hat{z} \cdot \cos \left|\theta_{t}\right|\right) .
$$

The Poynting vector points to the propagation direction of an electromagnetic wave and the direction of the power flow. Moreover, considering the magnetic and electric fields for the perpendicular polarization of the Figure 2.9 and using the Equation 2-80 to calculate the incident, reflected and transmitted Poynting vectors is possible to find the following relations

$$
\begin{gathered}
\mathbf{S}_{i}=\frac{1}{2} \frac{\left|E_{0}\right|^{2}}{\eta_{1}}\left(\hat{x} \cdot \sin \theta_{i}+\hat{z} \cdot \cos \theta_{i}\right), \\
\mathbf{S}_{r}=\frac{1}{2} \frac{\left|\Gamma E_{0}\right|^{2}}{\eta_{1}}\left(\hat{x} \cdot \sin \theta_{i}-\hat{z} \cdot \cos \theta_{i}\right), \\
\mathbf{S}_{t}=\frac{1}{2} \frac{\left|\mathrm{T} E_{0}\right|^{2}}{\eta_{2}}\left(-\hat{x} \cdot \sin \left|\theta_{t}\right|+\hat{z} \cdot \cos \left|\theta_{t}\right|\right) .
\end{gathered}
$$

Note that in medium 1 the wave vector and Poynting vectors, $\mathbf{k}_{i}$ and $\mathbf{S}_{i}$ point to the same direction, as well as $\mathbf{k}_{r}$ and $\mathbf{S}_{r}$, as expected for a DPS medium. On the other hand, in the medium 2 , the directions of $\mathbf{k}_{t}$ and $\mathbf{S}_{t}$ are antiparallel to each other. This phenomenon, as aforementioned, occurs in DNG media, which means that the wave propagates in a direction that opposes to the direction of the phase vector or the phase velocity.

Due to the fact that is not possible to find a DNG medium in natural form and its counterintuitive characteristics, the negative index of refraction can be scary at first. So, Dolling et al. developed photorealistic images using ordinary objects but with fictitious refractive index, providing a visual understanding of a DNG medium [25]. Figure 2.10 shows the images of two glasses filled with water. Besides that, the index of refraction of the water is 1.33 , but in one of the glasses the water has a fictitious refractive index of -1.33 , as it can be observed in Figure 2.10(b).

\subsection{4}

\section{Purely Left-Handed Transmission Line}

A purely left-handed transmission line (LHTL) can be considered the dual model of a conventional right-handed transmission line (RHTL). The LHTL presents backward wave propagation and is constituted of series timesunit-length capacitance $C_{L}^{\prime}$ and shunt times-unit-length inductance $L_{L}^{\prime}$, as it can be seen in Figure 2.11(a). The capacitive impedance and inductive admittance of the LHTL are written, respectively, as follow

$$
Z^{\prime}(\omega)=\frac{1}{j \omega C_{L}^{\prime}},
$$




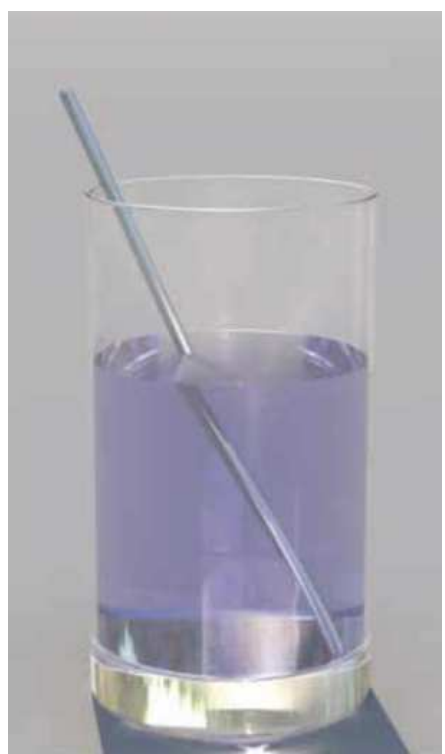

(a)

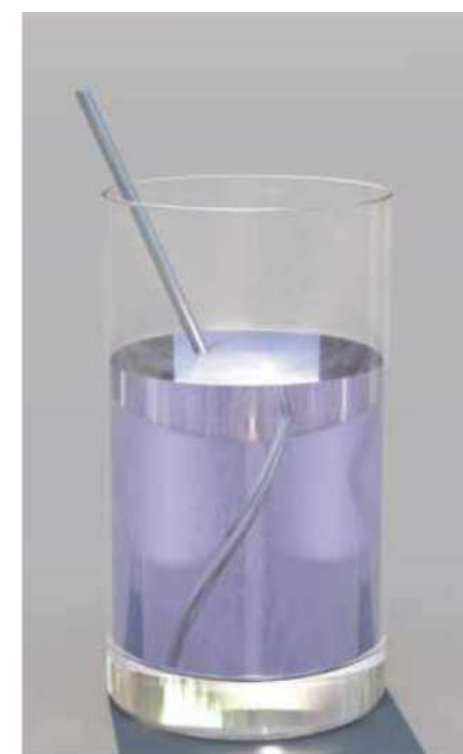

(b)

Figure 2.10: Photorealistic images of a drinking glass filled with water with its real index of refraction (a) and the same drinking glass with fitcious index of refraction equals to the water but negative (b) [25].

$$
Y^{\prime}(\omega)=\frac{1}{j \omega L_{L}^{\prime}} .
$$

The considered LHTL is lossless and its complex constant propagation is represented as

$$
\gamma(\omega)=j \beta(\omega)=\sqrt{Z^{\prime}(\omega) Y^{\prime}(\omega)}
$$

which leads to

$$
\beta(\omega)=\frac{-1}{\omega \sqrt{L_{L}^{\prime} C_{L}^{\prime}}}<0 .
$$

Differently from a conventional RHTL, the phase constant in LHTL is negative, which implies different effects in the propagating wave within the line at issue. In order to understand these effects, the phase velocity and group velocity in an LHTL are written, respectively, as

$$
\begin{gathered}
v_{p}=\frac{\omega}{\beta(\omega)}=-\omega^{2} \sqrt{L_{L}^{\prime} C_{L}^{\prime}}<0, \\
v_{g}=\left(\frac{\partial \beta(\omega)}{\partial \omega}\right)^{-1}=\omega^{2} \sqrt{L_{L}^{\prime} C_{L}^{\prime}}>0 .
\end{gathered}
$$

It can be observed that the phase and group velocities have opposite signs, which means that the direction of the propagating wave is antiparallel to the direction of the phase vector. This kind of wave is known as a backward or lefthanded propagating wave, which is the main feature of an LHTL, attracting the attention of researchers due to its exotic behavior. 


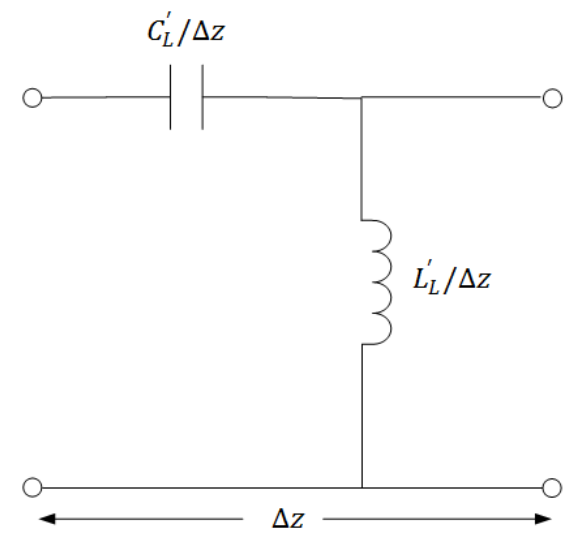

(a)

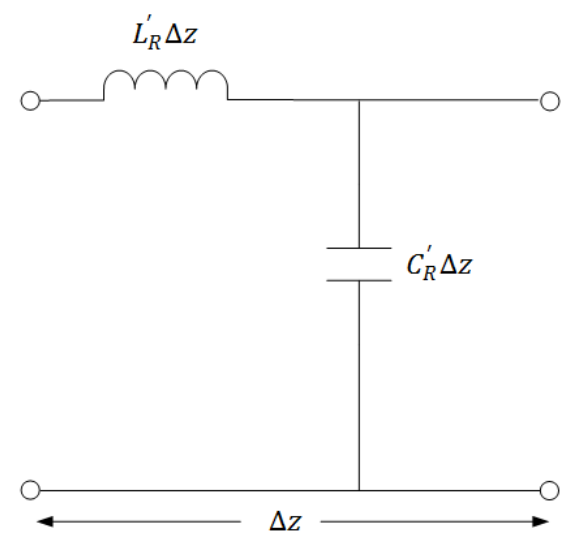

(b)

Figure 2.11: Homogeneous left- (a) and right-handed (b) transmission lines.

\subsection{5}

\section{Composite Right/Left-Handed Transmission Line}

The purely left-handed transmission line presents interesting characteristics but it is not possible to implement it due to the inherent effects of the transmission line. To be more specific, considering the LHTL structure in Figure 2.11(a), the current that flows through $C_{L}^{\prime}$ induces a magnetic field, so a series inductance $L_{R}^{\prime}$ must be taken into account. Moreover, the proximity of the two metal wires requires the inclusion of a shunt capacitor $C_{R}^{\prime}$. It is worth pointing out that the subscripts $L$ and $R$ refer to the inherent lumped elements of the left-handed and right-handed transmission lines, respectively.

In 2003, Caloz et al. coined the term composite right/left-handed (CRLH) transmission line in [26], in which the unavoidable effects of the right-handed TL were considered to provide an accurate and physically realizable transmission line approach of an LH medium. Furthermore, the equivalent circuit of a lossless and an effectively homogeneous CRLH TL is shown in Figure 2.12, and the per-unit-length impedance $Z^{\prime}(\Omega / \mathrm{m})$ and the per-unit-length admittance $Y^{\prime}(\mathrm{S} / \mathrm{m})$ are expressed as

$$
\begin{aligned}
& Z^{\prime}(\omega)=j\left(\omega L_{R}^{\prime}-\frac{1}{\omega C_{L}^{\prime}}\right), \\
& Y^{\prime}(\omega)=j\left(\omega C_{R}^{\prime}-\frac{1}{\omega L_{L}^{\prime}}\right) .
\end{aligned}
$$

The phase constant of the lossless CRLH TL is written as follows

$$
\beta=-j \sqrt{Z^{\prime} Y^{\prime}}=s(\omega) \sqrt{\frac{\omega^{2}}{\omega_{R}^{2}}\left(1-\frac{\omega_{s}^{2}}{\omega^{2}}\right)\left(1-\frac{\omega_{p}^{2}}{\omega^{2}}\right)},
$$

where $\omega_{R}=\left(L_{R}^{\prime} C_{R}^{\prime}\right)^{-\frac{1}{2}}, \omega_{s}=\left(L_{R}^{\prime} C_{L}^{\prime}\right)^{-\frac{1}{2}}, \omega_{p}=\left(L_{L}^{\prime} C_{R}^{\prime}\right)^{-\frac{1}{2}}$ and 


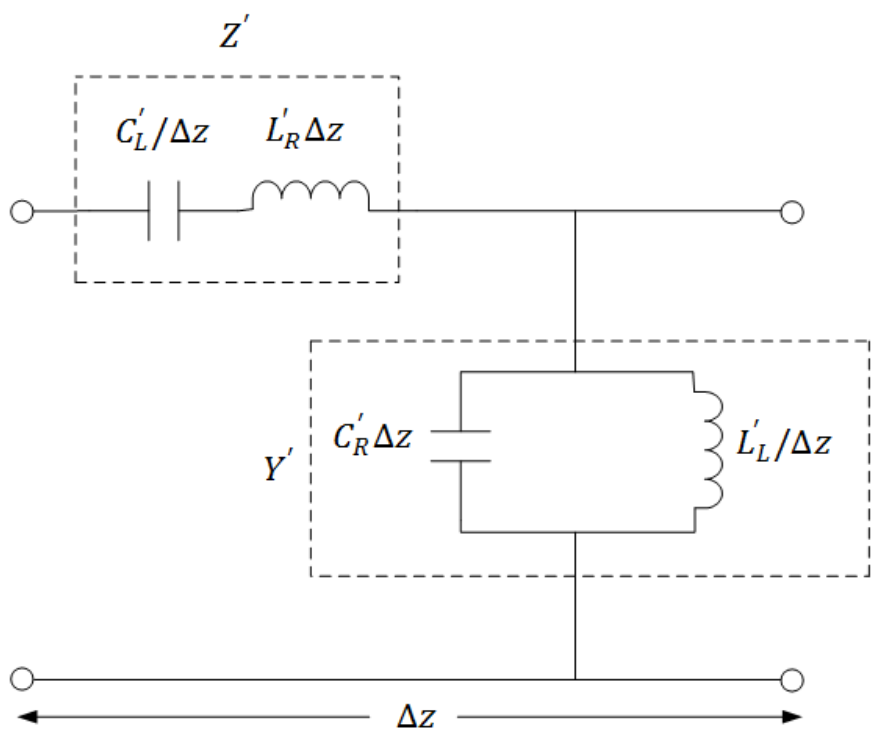

Figure 2.12: Effectively homogeneous CRLH transmission line.

$$
s(\omega)= \begin{cases}-1 & \text { if } \omega<\min \left(\omega_{s}, \omega_{p}\right) \\ +1 & \text { if } \omega>\max \left(\omega_{s}, \omega_{p}\right)\end{cases}
$$

where the positive sign denotes the behaviour of a RHTL and the negative sign, an LHTL. So, the dispersion diagram of the CRLH TL can expressed, as it can be seen in Figure 2.13. The phase velocity $\left(v_{p}=\frac{\omega}{\beta(\omega)}\right)$ and the group velocity $\left(v_{g}=\left(\frac{\partial \beta(\omega)}{\partial \omega}\right)^{-1}\right)$ can be calculated from the dispersion diagram. As aforementioned, for a purely LHTL, the phase and group velocities are antiparallel, $\left(v_{p} v_{g}<0\right)$. On the other hand, the phase and group velocities are parallel for a purely RHTL, $\left(v_{p} v_{g}>0\right)$. Furthermore, the dispersion diagram of the CRLH TL shows an LH and an RH region, in which the boundary between these two regions is located around the frequency of maximum attenuation $\omega_{0}$, that can be find through the root of the derivative of the phase constant

$$
\frac{\partial \beta}{\partial \omega}=s(\omega) \frac{\omega / \omega_{R}^{2}-\omega_{L}^{2} / \omega^{3}}{\sqrt{\left(\omega / \omega_{R}\right)^{2}+\left(\omega_{L} / \omega\right)^{2}-\omega_{L}^{2} / \omega_{s}^{2}-\omega_{L}^{2} / \omega_{p}^{2}}}=0,
$$

obtaining

$$
\omega_{0}=\frac{1}{\sqrt[4]{L_{R}^{\prime} C_{R}^{\prime} L_{L}^{\prime} C_{L}^{\prime}}},
$$

where

$$
\omega_{L}=\frac{1}{\sqrt{L_{L} C_{L}}} .
$$

Depending on the frequency range, the effective permittivity and permeability can be positive or negative and can be represented, respectively, as

$$
\epsilon_{\mathrm{eff}}(\omega)=C_{R}^{\prime}-\frac{1}{\omega^{2} L_{L}^{\prime}},
$$




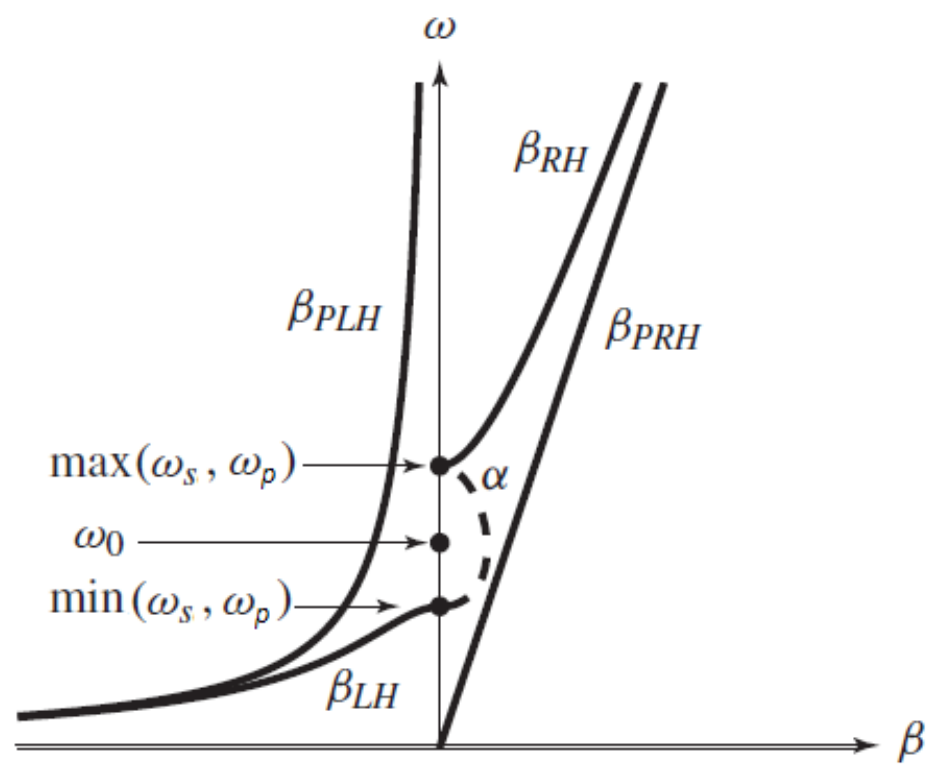

Figure 2.13: Dispersion diagram of the purely $\mathrm{RH}\left(\beta_{\mathrm{PRH}}\right)$, the purely $\mathrm{LH}$ $\left(\beta_{\mathrm{PLH}}\right)$ and CRLH $\left(\beta_{\mathrm{RH}}\right.$ and $\left.\beta_{\mathrm{LH}}\right)$ TLs, considering the power flow along the $+z$ direction. [26].

$$
\mu_{\mathrm{eff}}(\omega)=L_{R}^{\prime}-\frac{1}{\omega^{2} C_{L}^{\prime}}
$$

One of the most important quantities of the transmission lines is the characteristic impedance, which is defined, for the CRLH TL, as

$$
Z_{c}=\sqrt{\frac{L_{L}^{\prime}}{C_{L}^{\prime}}} \sqrt{\frac{\left(\omega / \omega_{s}\right)^{2}-1}{\left(\omega / \omega_{p}\right)^{2}-1}} .
$$

Besides the characteristic impedance of the CRLH TL, other parameters are essential to the analysis of a TL, which are the guided wavelength $\lambda_{g}$, phase velocity $v_{p}$, and group velocity $v_{g}$, and they are expressed as

$$
\begin{gathered}
\lambda_{g}=\frac{2 \pi}{|\beta|}=\frac{2 \pi}{\sqrt{\left(\omega / \omega_{R}\right)^{2}+\left(\omega_{L} / \omega\right)^{2}-\omega_{L}^{2} / \omega_{s}^{2}-\omega_{L}^{2} / \omega_{p}^{2}}}, \\
v_{p}=\frac{\omega}{\beta}=s(\omega) \frac{\omega}{\sqrt{\left(\omega / \omega_{R}\right)^{2}+\left(\omega_{L} / \omega\right)^{2}-\omega_{L}^{2} / \omega_{s}^{2}-\omega_{L}^{2} / \omega_{p}^{2}}}, \\
v_{g}=\left(\frac{\partial \beta}{\partial \omega}\right)^{-1}=\frac{\left|\omega \omega_{R}^{-2}-\omega^{-3} \omega_{L}^{2}\right|}{\sqrt{\left(\omega / \omega_{R}\right)^{2}+\left(\omega_{L} / \omega\right)^{2}-\omega_{L}^{2} / \omega_{s}^{2}-\omega_{L}^{2} / \omega_{p}^{2}}} .
\end{gathered}
$$

Therefore, the CRLH TL is an accurate model of LH media, once a purely LHTL does not physically exist. Even though the quantities of the CRLH TL seem more complicated than the ones for the conventional RHTL, all of these 
quantities are derived from the classical transmission line theory.

\subsection{6 \\ Metasurface}

A metasurface is considered the surface equivalent of a metamaterial, where its thickness is much smaller than the operating wavelength and can be interpreted as an infinitesimal sheet that provides changes in the phase and amplitude of the scattered fields. Moreover, the metasurface can be divided into two broad categories: metafilm and metascreen. A metafilm refers to a metasurface in which its scatter elements of a unit cell are not touching the scatters of an adjacent cell. On the other hand, metasurfaces with the scatter of adjacent unit cells in touch with each other are known as metascreens [27].

Considering that this work is regarding electromagnetic-wave absorbers based on metafilms, as it will be noted in the following chapters, it is important to understand the differences between a metasurface and a frequency selective surface (FSS). This is a relevant issue once a great number of absorbers are classified as FSS and rely on concepts of it, and they are both periodic structures.

The classification and behavior of a composite periodic two-dimensional structure depend on the dimensions of their scatters and/or lattice with respect to the operating wavelength. To begin with, the periodicity of FSSs is usually comparable to or smaller than the operating wavelength. Moreover, the lattice of the FSS determines in which frequency the structure resonates [27]. In this kind of structure, the presence of high-order Floquet-Bloch modes interferes even more in the fundamental propagating wave as the lattice is getting comparable to the wavelength.

The metasurface is a two-dimensional composite material in which its periodicity is much smaller than the wavelength. Also, the resonance depends on the shape of the scatters, and not on the lattice by itself. So, they are designed in a way that the very scatters resonate. Furthermore, a metasurface can be considered a dispersive medium, but it is possible to find effective permittivity, permeability and refractive index, for instance.

To summarize, metasurfaces and FSSs differ from each other in the part of their structure that is more relevant to the resonance at a given frequency. The resonance in FSSs depends on their periodicity, while the resonance in metasurfaces is mainly related to the scatters by themselves. Moreover, unlike the FSSs, the high-order Floquet-Bloch modes are not relevant when it comes to the metasurfaces, once their periodicity is much smaller than the operating wavelength. A metasurface can be understood as some type 
of effective medium, which is not true for FSSs.

\subsection{7}

\section{Characterizing a Metasurface}

When it comes to the constitutive electromagnetic parameters retrieval, there is a large number of available methods according to some features of the sample and the classification of the metamaterial at issue. Usually, the constitutive parameters are calculated in terms of either measured or numerically calculated complex scattering parameters $S_{11}$ and $S_{21}$.

One of the main mistakes regarding the modeling of metamaterials is the use of the same method for both full 3D metamaterials and metasurfaces. To be more specific, the method of Nicolson-Ross-Weir (NRW) [28], [29] is often wrongly employed in metasurfaces. This method, for example, is appropriate for bulk metamaterials and not for metasurface, and this incorrect employment leads to results that depend on the sample thickness of the metasurface, which is fundamentally wrong. The permittivity and permeability of a bulk metamaterial must be the same regardless of the thickness of the sample. Therefore, the use of NRW or similar methods in metasurface leads to results that vary with the thickness of the metasurface, which means that the effective material properties are not uniquely defined. Moreover, as the thickness of the metamaterial is getting much smaller than the operating wavelength, i.e. a metasurface, the localized effects due to boundaries are getting too relevant to be ignored [30].

Throughout this work, all the proposed structures are classified as metafilms. The effective medium method employed in metamaterial to retrieve the material properties is not suitable for this type of metasurface. For this kind of structure the generalized sheet transition conditions (GSTCs) is more appropriated [31]. This method uses the surface susceptibilities to describe the metafilms, once these parameters are uniquely defined for them. Moreover, the transverse surface susceptibilities for TE-polarized waves are written as

$$
\begin{gathered}
\chi_{\mathrm{MS}}^{x x}=\frac{2 j}{k_{0}} \frac{S_{11}(0)-S_{21}(0)+1}{S_{11}(0)-S_{21}(0)-1}, \\
\chi_{\mathrm{ES}}^{y y}=\frac{2 j}{k_{0}} \frac{S_{11}(0)+S_{21}(0)-1}{S_{11}(0)+S_{21}(0)+1}, \\
\chi_{\mathrm{MS}}^{z z}=-\frac{\chi_{\mathrm{ES}}^{y y}}{\sin ^{2} \theta}+\frac{2 j \cos \theta}{k_{0} \sin ^{2} \theta} \frac{R(\theta)+T(\theta)-1}{R(\theta)+T(\theta)+1},
\end{gathered}
$$

where $\chi_{\mathrm{MS}}^{x x, y y, z z}$ and $\chi_{\mathrm{ES}}^{x x, y y, z z}$ are the magnetic and electric transverse surface susceptibilities, respectively, $S_{11}(0)$ and $S_{21}(0)$ are the complex reflection and transmission coefficients under normal incidence, respectively, and $R(\theta)$ and $T(\theta)$ are the complex reflection and transmission coefficients at a given angle 
of incidence $\theta$. Moreover, for TM-polarization wave, the surface susceptibilities are

$$
\begin{gathered}
\chi_{\mathrm{ES}}^{x x}=\frac{2 j}{k_{0}} \frac{S_{11}(0)+S_{21}(0)-1}{S_{11}(0)+S_{21}(0)+1}, \\
\chi_{\mathrm{MS}}^{y y}=\frac{2 j}{k_{0}} \frac{S_{11}(0)-S_{21}(0)+1}{S_{11}(0)-S_{21}(0)-1}, \\
\chi_{\mathrm{MS}}^{z z}=-\frac{\chi_{\mathrm{MS}}^{y y}}{\sin ^{2} \theta}+\frac{2 j \cos \theta}{k_{0} \sin ^{2} \theta} \frac{T(\theta)-1-R(\theta)}{T(\theta)+1-R(\theta)} .
\end{gathered}
$$

All samples analysed in this work are metal-backed, which means that $S_{21}$ is always null and present polarization insensitivity, considering the two orthogonal polarization TE and TM, and, thus, $\chi_{\mathrm{ES}}^{x x}=\chi_{\mathrm{ES}}^{y y}$ and $\chi_{\mathrm{MS}}^{x x}=\chi_{\mathrm{MS}}^{y y}$. Considering these features, the susceptibilities can be simplified [32] and written as

$$
\begin{aligned}
\chi_{e s} & =\frac{2 j}{k_{0}} \frac{S_{11}(0)-1}{S_{11}(0)+1}, \\
\chi_{m s} & =\frac{2 j}{k_{0}} \frac{S_{11}(0)+1}{S_{11}(0)-1} .
\end{aligned}
$$

Moreover, there are connections between the susceptibilities and the effective permittivity and permeability, which are valid and useful only for metasurfaces located on the plane $x y$ and $z=0$, and are expressed as

$$
\begin{aligned}
& \epsilon_{e f f}=1+\frac{\chi_{e s}}{d}, \\
& \mu_{e f f}=1+\frac{\chi_{m s}}{d},
\end{aligned}
$$

where $d$ is the thickness of the metasurface.

The experimental characterization of material or metamaterial requires more attention to some details [33]. The first one is regarding the sample size, and in order to have accurate results, the sample size should be at least twice larger than the operating wavelength. Furthermore, the far-field requirement must be considered as well, that is ruled by the following relation

$$
r>\frac{2 D^{2}}{\lambda}
$$

where $r$ is the distance between the antenna aperture and the sample, $D$ is the largest dimension of the antenna aperture, and $\lambda$ is the operating wavelength. The fulfilling of this requirement ensures that the impinging electromagnetic wave can be treated as a plane wave from the perspective of the device under test. It is important to point out that the far-field requirement must be calculated for both device under test and the reference antenna, and the biggest value of them must be used. Moreover, Figure 2.14 illustrates a typical freespace measurement setup using reflection method to calculate the reflection 
coefficient from the material sample.

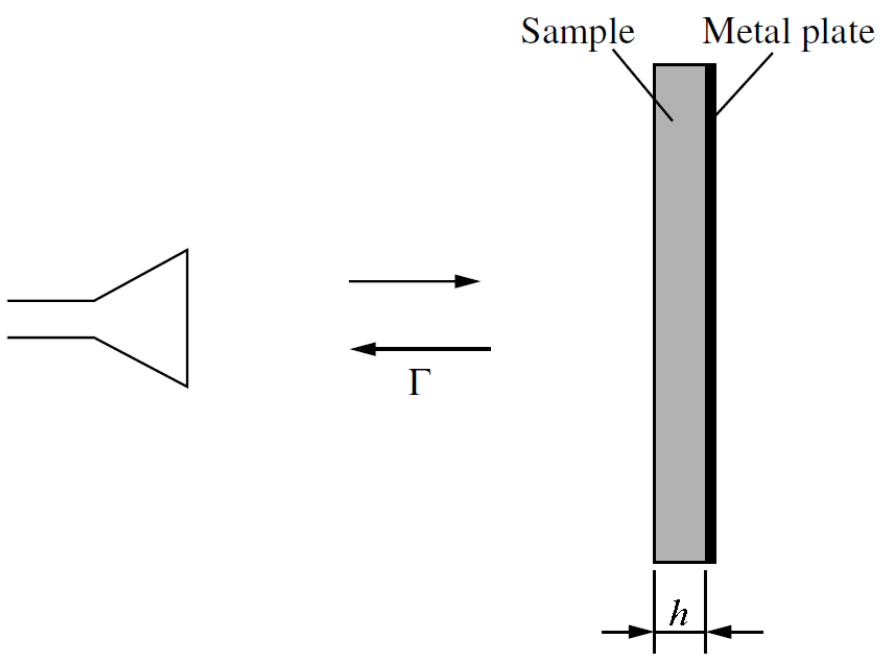

Figure 2.14: Free-space measurement of a material sample [33].

\section{8}

\section{Radar Cross-Section}

According to the IEEE Standard Dictionary of Electrical and Electronic Terms [34], the expression radar cross-section (RCS) means "the measure of reflective strength of a target defined as $4 \pi$ times the ratio of the power per unit solid angle scattered in a specified direction to the power per unit area in a plane wave incident on the scatterer from a specified direction". In other words, RCS can be summarized as the ability of a target to reflect the radar signals in the direction of the receiver antenna. Furthermore, the RCS is very useful to the evaluation of absorbers and has been extensively studied since the advent of stealth concept in radar systems, which the main goal is to attenuate the scattered power to below the threshold of the radar receiver.

Electromagnetic-wave absorbers are extremely important in radar systems and are used to decrease the RCS of a target. Absorbers can help an object to become stealth to the radar, which is quite useful in military applications. Taking this into account, a great number of low-cost and low-profile absorbers aiming the reduction of RCS has been studied and developed, which can be used in satellite, ships, and aircraft, for instance. The materials employed for the reduction of RCS are known as radar absorbing materials (RAM). Furthermore, it is important to note that the development of efficient RAMs, such as absorbers, contributed to the technological advance of radar systems. This is because the progress of RAM automatically demands the creation of more sensitive radar systems in order to counterbalance the RCS reduction. 
It is worth pointing out that there are two main types of RCS: monostatic and bistatic. In the monostatic RCS, both transmission and reception are provided by the same antenna. However, when the transmitter and receiver antennas are slightly separated from each other, the radar systems is called quasi monostatic, which is the most common kind of radar system. For the sake of simplicity, throughout this work, the quasi monostatic and monostatic are considered the same kind of radar system. On the other hand, in the bistatic RCS, both transmitter and receiver antennas are placed considerably apart from each other. Moreover, these two configurations of radar systems are shown in Figure 2.15.

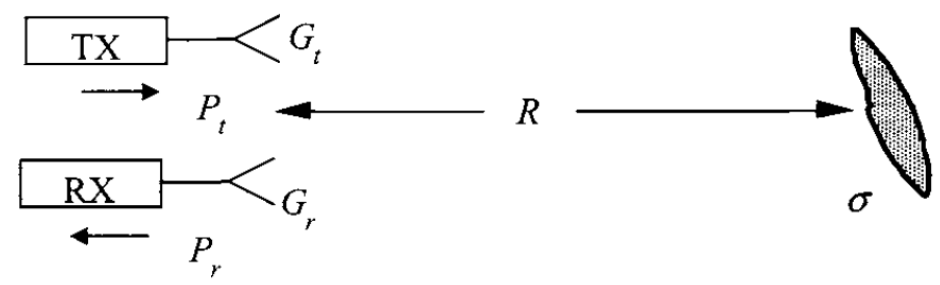

(a)

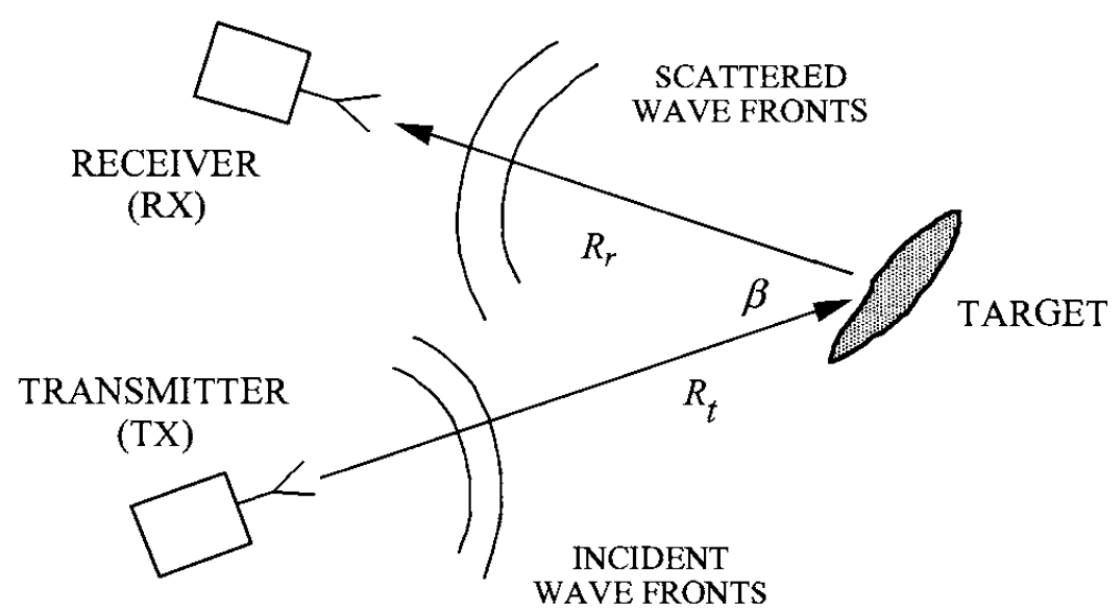

(b)

Figure 2.15: Radar Cross Section: monostatic (a) and bistatic (b) [35].

To describe mathematically the RCS, $\sigma$, the monostatic radar is considered. So, the power density of the incident wave $S_{i}$ can be written as

$$
S_{i}=\frac{E_{i} H_{i}}{2}=\frac{Y_{0}\left|E_{i}\right|^{2}}{2}
$$

where $E_{i}$ and $H_{i}$ are the magnitudes of the incident electric and magnetic fields on the target, respectively, and $Y_{0}$ is the free-space admittance. Moreover, it is assumed that the target scatters the wave and can be considered some sort of effective area $\sigma$, as it is done when it comes to antennas [36]. Therefore, the captured power $P$ can be expressed as 


$$
P=\sigma S_{i}=\frac{\sigma Y_{0}\left|E_{i}\right|^{2}}{2}
$$

Considering that the target scatters the captured power isotropically and that the distance between the target and the antenna is much bigger than the size of the target, the scattered power density $S_{s}$ can be represented in terms of the captured power and the area of a sphere of radius $r$,

$$
S_{s}=\frac{P}{4 \pi r^{2}}=\frac{\sigma Y_{0}\left|E_{i}\right|^{2}}{8 \pi r^{2}} .
$$

Also, as for $S_{i}$, the scattered power density can be written in terms of the scattered electric and magnetic fields strength,

$$
S_{s}=\frac{E_{s} H_{s}}{2}=\frac{Y_{0}\left|E_{s}\right|^{2}}{2} .
$$

The RCS $\sigma$ can be expressed in terms of the scattered and incident electric fields strengths solving Equations 2-121 and 2-122,

or

$$
\sigma=4 \pi r^{2} \frac{\left|E_{s}\right|^{2}}{\left|E_{i}\right|^{2}}=4 \pi r^{2} \frac{\left|H_{s}\right|^{2}}{\left|H_{i}\right|^{2}},
$$

$$
\sigma=4 \pi r^{2} \frac{S_{s}}{S_{i}} .
$$

This equation matches with the definition of RCS showed at the beginning of this Section.

The dependence of the distance $r$ in the RCS measurement implies that the target characteristic is a function of the measurement environment. To minimize this issue, the value of $r$ is forced to approach the infinity, and, thus, the standard equation of the RCS is defined as

$$
\sigma=4 \pi \lim _{r \rightarrow \infty} r^{2} \frac{\left|E_{s}\right|^{2}}{\left|E_{i}\right|^{2}}=4 \pi \lim _{r \rightarrow \infty} r^{2} \frac{\left|H_{s}\right|^{2}}{\left|H_{i}\right|^{2}} .
$$

Equation 2-126 represents the standard definition of RCS, which its unit is length square. Furthermore, the RCS can be expressed in decibels relative to a square meter $\left(\mathrm{dBm}^{2}\right.$ or $\left.\mathrm{dBsm}\right)$, which is calculated as

$$
\sigma[\mathrm{dBsm}]=10 \log \left(\sigma\left[\mathrm{m}^{2}\right]\right) .
$$

The RCS can be correlated to some parameters of the antennas such as gain $G$, transmitted power $P_{T}$, and received power $P_{R}$, and forming the radar equation [37], which is expressed as

$$
P_{R}=\frac{P_{T} G^{2} \sigma \lambda^{2}}{(4 \pi)^{3} r^{4}} .
$$

The relation between the transmitted and received power relies on the physical features of the target such as its geometry, and electrical and magnetic properties. In addition, the polarization and frequency of the radar influence 
the relation between $P_{T}$ and $P_{R}$.

There is a conceptual definition of RCS that provides an easier understanding of its meaning and consists of a product of three factors: projected cross-section, reflectivity, and directivity.

$$
\sigma=\text { Projected cross section } \times \text { Reflectivity } \times \text { Directivity }
$$

where the projected cross-section is the aperture of the target seen by the radar, reflectivity is the intercepted radiated power by the target, and the directivity is the ration of the backscattered power into the radar's direction to the power that would have been scattered isotropically.

Some ordinary shapes already present well-defined equations to describe their RCS, as can be seen in Table 2.2. The equations showed in this Table are valid in the optical region, which is the region where the dimensions of the target are larger than the operating wavelength.

Besides the cross-section presented so far, there are other types of it, such as absorption cross-section (ACS), which is used in a non-conducting target that not only reflects the incident wave but also absorbs part of it. The ACS $\sigma_{a}$ is expressed as

$$
\sigma_{a}=\frac{P_{a}}{S_{i}}
$$

where $P_{a}$ is the absorbed power by the target and $S_{i}$ is the incident power density. The absorbed power can be determined via analytical methods using the resistivities and currents of the target at issue.

In conclusion, among all the types of cross-sections, the RCS is, by far, the most important and popular. Besides that, it is an important tool to provide an insight into the performance of an electromagnetic-wave absorber or RAM. The importance of absorber materials in radar systems is an important indicator of how essential this kind of material is in nowadays applications. 
Table 2.2: RCS of some ordinary shapes in the optical region.

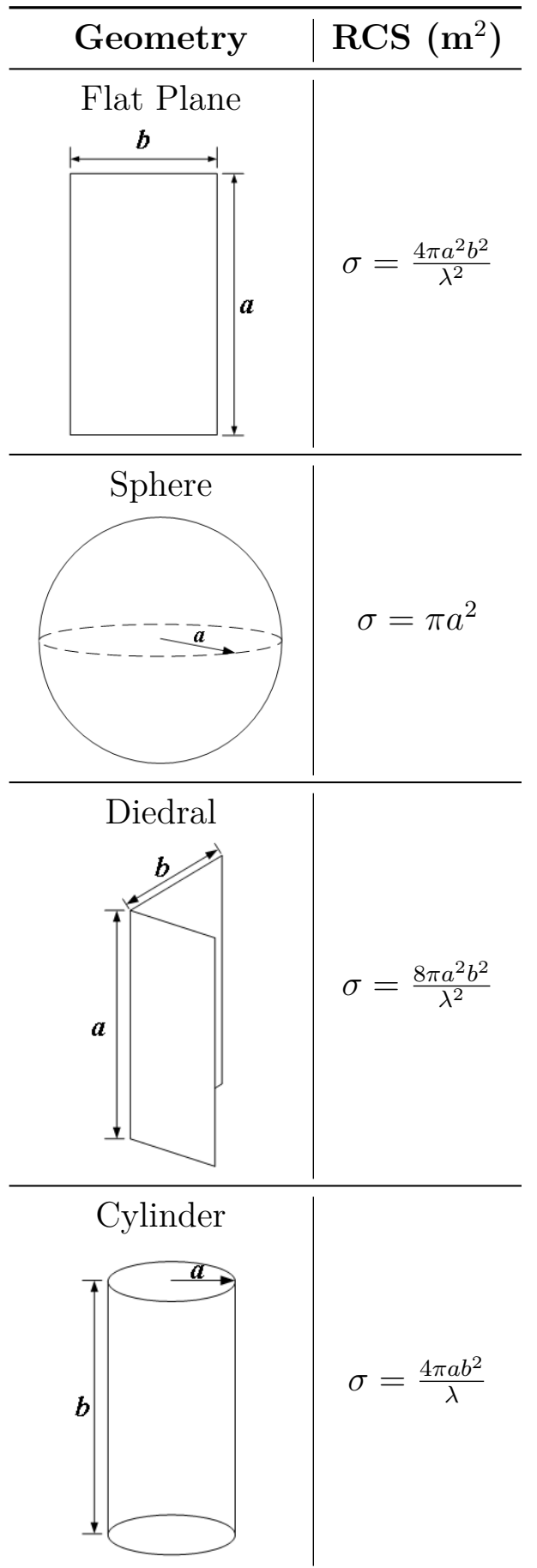




\section{3 \\ Electromagnetic-Wave Absorber}

In this Chapter, the theory and main aspects necessaries to the comprehension of electromagnetic wave absorbers are presented as well as their precursors and categories. In addition, theorems regarding some limitations of absorbers are detailed, and methods to interpret and characterize them are introduced.

\section{1}

\section{Introduction}

Once the number of wireless devices has been increasing, techniques and methods have been proposed in order to reduce spurious radiation or sidelobe radiation, for instance. In this scenario, electromagnetic-wave absorbers are applied to deal with it and can find many applications in civilian and military issues.

Basically, an electromagnetic-wave absorber, or simply absorber, is a structure or material that attenuates the energy of an impinging EM wave. The efficiency of absorbers is usually given in terms of their curve of absorptivity $A(\omega)$ and it is expressed by

$$
A(\omega)=1-R(\omega)-T(\omega),
$$

where $R(\omega)$ is the reflectance and $T(\omega)$ is the transmittance. Researchers have been working in order to obtain an absorptivity as near as possible of unity at the range of frequency of interest.

\section{2}

\section{Types of Electromagnetic-Wave Absorbers}

Absorbers can be divided into two main categories: resonant and broadband absorbers. Basically, resonant absorbers are structures that resonate due to the interaction with the impinging radiation in a specific frequency. Broadband absorbers are structures that can attenuate the reflection in a wide frequency band, and, usually, their properties are frequency independent. The details of the structure of these two types of absorbers and how they operate is analyzed in the following. 


\subsection{1}

\section{Resonant Absorbers}

Resonant absorbers can present a single layer or multiple layers in which each layer has a quarter of the operating wavelength of thickness. The absorption in this kind of structure is supported by the transmission line theory, which says that a short circuit, i.e. a metal plate, placed a quarterwavelength of a load will act as an open circuit. Thereby, the impedance of the resonant absorber matches free-space, and, thus, the incident wave considers the absorber as being free-space and there is no reflection.

\subsubsection{1}

\section{Salisbury Screen}

One of the oldest forms of electromagnetic-wave absorbers is the Salisbury screen, which was invented by W. W. Salisbury in 1952 as part of an American project called Halpern Anti Radar Paint (HARP), and its structure consists of three layers: a purely resistive sheet, a lossless dielectric, and metal ground plane, as it can be seen in Figure 3.1(a).

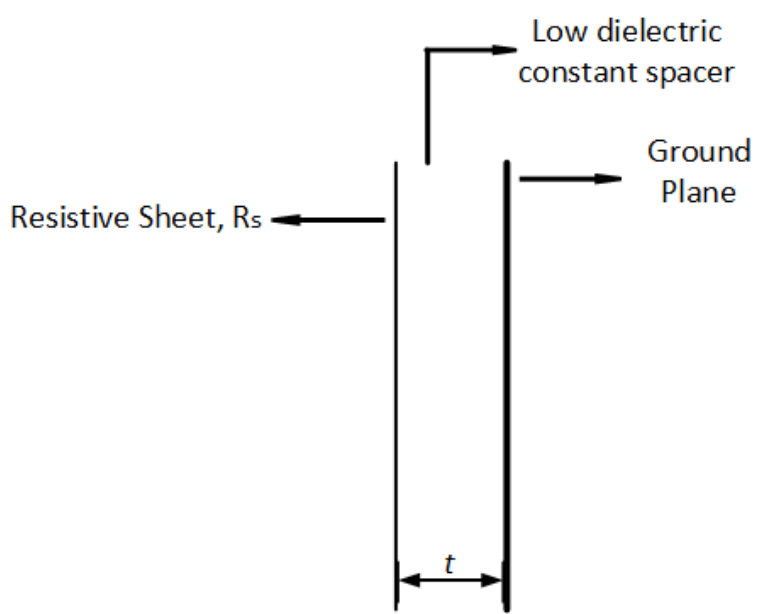

(a)

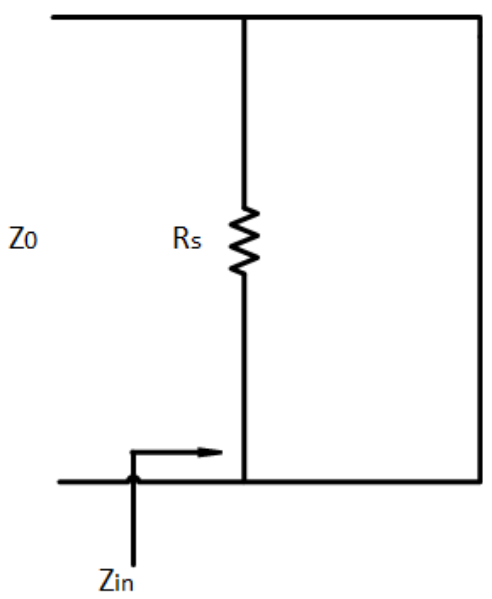

(b)

Figure 3.1: Salisbury Screen (a) and its equivalent circuit (b).

The resistive sheet and the ground plane are separated by a lossless dielectric that is $\frac{\lambda}{4}$, or odd multiple of $\frac{\lambda}{4}$, thick, and, therefore, the short is transformed into an open circuit at the resistive sheet at the resonance. Moreover, Figure 3.1(b) presents the equivalent circuit of the Salisbury screen. In order to achieve a zero reflection, the resistivity of the sheet is usually 377 $\Omega / \square$, as can be inferred from the following relation

$$
\frac{1}{Z_{\text {in }}}=\frac{1}{R_{s}}+\frac{1}{\infty}=\frac{1}{R_{s}} .
$$


The absorption in the Salisbury screen occurs due to cancellation of the waves reflected on the resistive sheet and the ground plane. To be more precise, when an impinging wave hits the $377 \Omega$ /square resistive sheet, there is no reflection at the sheet and the wave propagates through the dielectric. This wave travels $\frac{\lambda}{4}$ and it is reflected by the ground plane. Afterward, the reflected wave travels $\frac{\lambda}{4}$ again and eventually hits the resistive sheet, which implicates in a phase change of $180^{\circ}$ once the distance traveled was $\frac{\lambda}{2}$. Therefore, this wave is completely out of phase with the just incident EM wave, and, thus, resulting in a destructive interference between them.

It is easy to realize that the Salisbury screen is a narrowband device, once its resonance relies on the thickness of the structure. This feature implies that, for some applications, the Salisbury screen is considered bulky, which limits its usage.

\subsubsection{2}

\section{Jaumann Absorber}

Although it is considered an extension of the Salisbury screen, the Jaumann absorber was discovered independently in Germany and, at first, its main application was to camouflage submarine snorkels and periscopes. Additionally, in order to increase the bandwidth, the Jaumann absorber cascades several resistive sheets, and its structure and equivalent circuit can be seen in Figure 3.2.

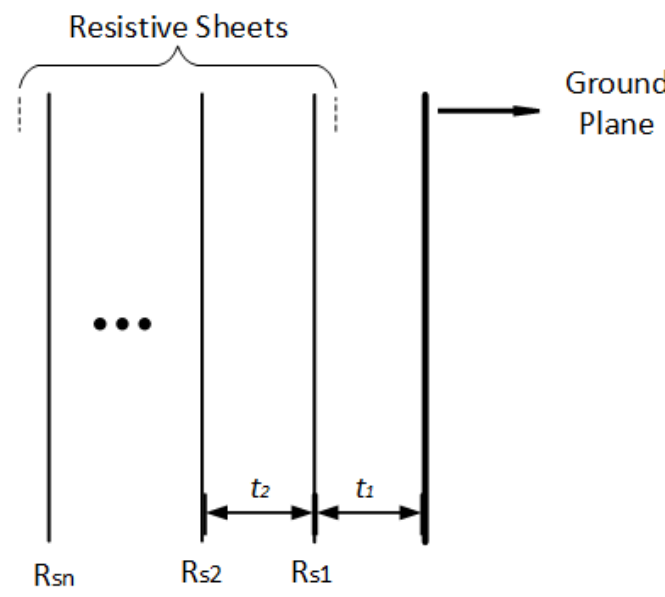

(a)

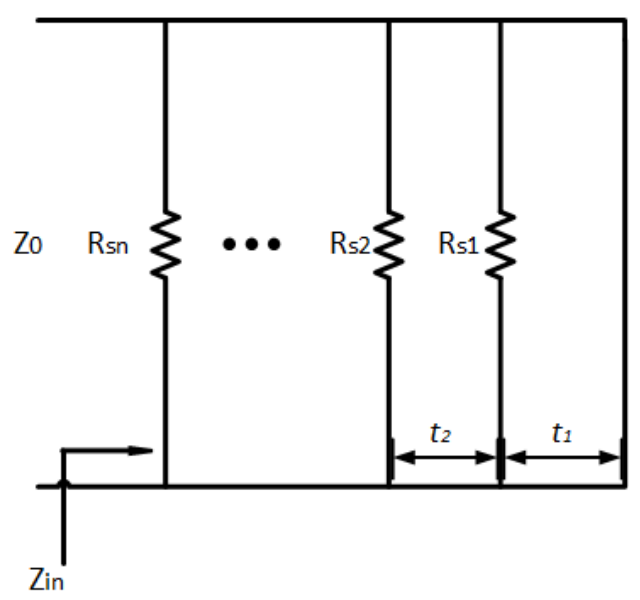

(b)

Figure 3.2: Jaumann absorber (a) and its equivalent circuit (b).

Each resistive sheet is designed to operate at a distinct operating wavelength, creating multiple peaks of absorption around the central wavelength $\left(\lambda_{0}\right)$; hence, the distance between the different sheets are not equal. Besides 
that, to optimal performance, the highest value of resistivity must be on the front and this value decreases gradually until the last sheet, that must present the lowest value of resistivity. The number of sheets depends on how large the bandwidth of absorption is, and, thus, the Jaumann absorber is usually quite thick.

\subsubsection{3}

\section{Dällenbach Layer}

The Dällenbach Layer presents a different approach to the previously mentioned absorbers. It consists in a homogeneous lossy layer in front of a metal plate, as it can be observed in Figure 3.3. The losses experimented by the electromagnetic wave that passes through the layer comes from the imaginary part of the permittivity and/or permeability of the material employed. So, it does not require the employment of a resistive sheet. By properly choosing the properties and the thickness of the material, it is possible to tune the frequency in which the peak of absorption occurs.

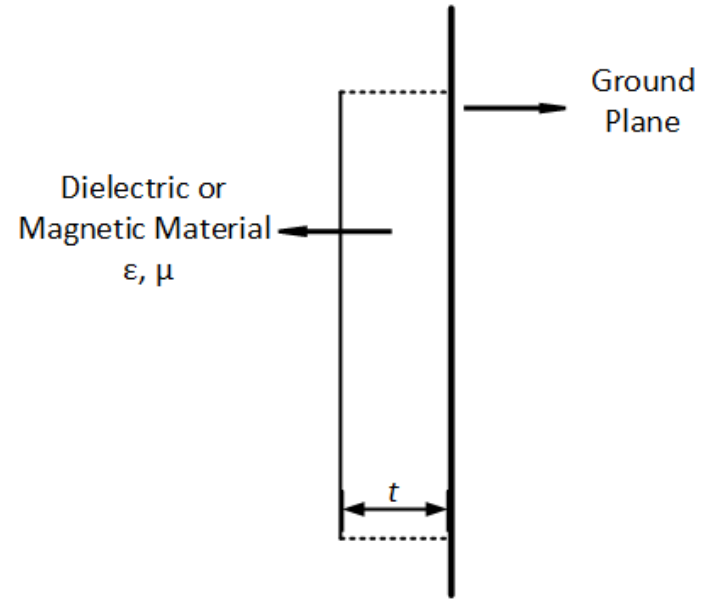

(a)

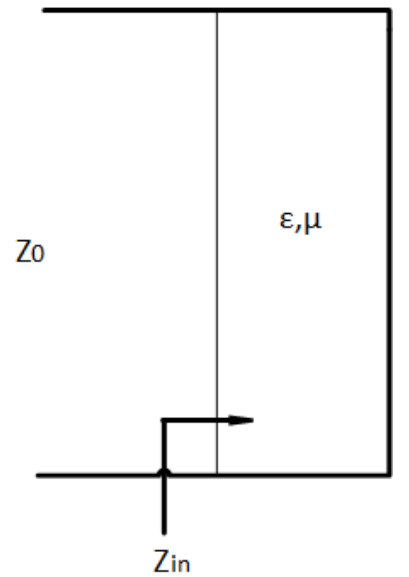

(b)

Figure 3.3: Dällenbach layer (a) and its equivalent circuit (b).

\subsubsection{4}

\section{Circuit Analog Absorber}

Circuit analog (CA) absorbers are another type of resonant absorber. The usual composition of it consists of a metallic geometric pattern etched on a lossless thin film. Unlike the Salisbury screen and the Jaumann absorber, which employ purely resistive sheets, the CA absorbers employ sheets that present a reactive part as well. The use of this kind of material provides great flexibility to the structure in terms of size, weight, bandwidth, and so on. The 
reactive part is achieved arranging geometric patterns periodically. Therefore, the input impedance of a CA absorber depends on the geometric pattern and the sheet resistance employed, and, thus, both of them can be tailored to achieve a high level of absorption over the frequency range of interest.

The performance of a CA absorber can be analyzed in terms of its lumped elements, making its design more intuitive. Usually, they are represented by a combination of RLC circuits and their geometries by themselves control the effective capacitance and inductance. Basically, any frequency selective surface (FSS) pattern can be used on a CA sheet, and examples of these elements are shown in Figure 3.4.
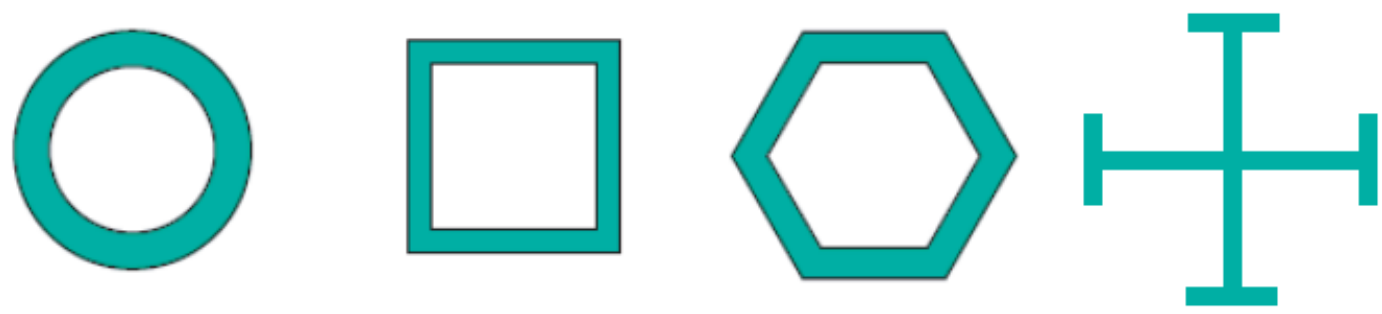

Figure 3.4: Examples of FSS elements [38].

\subsection{2}

\section{Broadband Absorbers}

The broadband absorbers, as the name implies, operate in a wider frequency band than the resonant absorbers. The most common example of this kind of absorber is the one used in anechoic chambers, as it can be seen in Figure 3.5. Additionally, pyramids or wedge shapes loaded with lossy materials are commonly used in order to create a slow transition from free-space to the lossy material. So, the electromagnetic wave is gradually absorbed over the length of the structure.

\subsection{3}

\section{Physical Mechanisms of Absorption}

There are three main physical mechanisms of absorption: ohmic loss, dielectric loss, and magnetic loss. Basically, ohmic loss occurs in imperfect conductors, and it is related to the motion of free charges, which are usually electrons.

To understand the concept of dielectric and magnetic losses, the complex relative permittivity and permeability must be considered first, and they are represented, respectively, by 


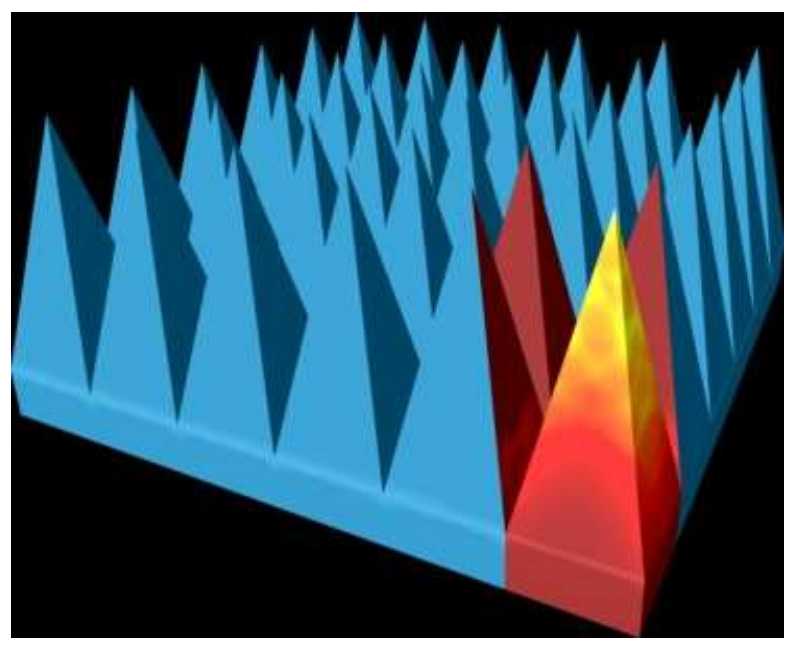

Figure 3.5: Broadband absorber used in anechoic chambers [39].

$$
\begin{aligned}
& \varepsilon_{r}=\varepsilon_{r}^{\prime}-j \varepsilon_{r}^{\prime \prime}, \\
& \mu_{r}=\mu_{r}^{\prime}-j \mu_{r}^{\prime \prime},
\end{aligned}
$$

where $\varepsilon_{r}^{\prime}$ is the lossless part of the permittivity of the material, $\varepsilon_{r}^{\prime \prime}$ represents the loss due to damping of the vibrating dipole moments, $\mu_{r}^{\prime}$ is the lossless part of the permeability of the material, and $\mu_{r}^{\prime \prime}$ denotes the losses due to damping forces. Hence, the imaginary parts of both complex relative permittivity and permeability are related to dielectric and magnetic losses, respectively. Moreover, the dielectric and magnetic losses are usually quantified in terms of electric and magnetic loss tangents which are expressed, respectively, as

$$
\begin{aligned}
\tan \delta_{\varepsilon} & =\frac{\varepsilon_{r}^{\prime \prime}}{\varepsilon_{r}^{\prime}}, \\
\tan \delta_{\mu} & =\frac{\mu_{r}^{\prime \prime}}{\mu_{r}^{\prime}} .
\end{aligned}
$$

These quantities above provide a good insight into how well the material provides losses to the propagating wave within its structure. However, it is worth pointing out that the dielectric loss is much stronger than the ohmic loss in most of the absorbers [40].

Researchers use to plot the current surface density in the conductor on the top and on the bottom of an absorber at the resonance frequency in order to find evidence of magnetic resonant responses. To make a long story short, the presence of circulating current loop in the absorber indicates a strong magnetic resonance within the structure. In addition, the electric resonance is determined by the electric-like dipole resonance frequency of the absorber [41]. Therefore, both electric and magnetic resonances are able, together or not, to 
create the electromagnetic absorption.

\section{3}

\section{Theorems for Electromagnetic-Wave Absorber}

\subsection{1}

Weston's Theorems

Weston postulated two theorems regarding the backscattering fields from electromagnetic-wave absorbers, which are essentially important in absorber theory [42]. Moreover, these theorems are derived from Maxwell's equation and the boundary conditions and they are presented hereafter.

Theorem 1: If a plane electromagnetic wave is incident on a body composed of a material such that $\frac{\mu}{\mu_{0}}=\frac{\epsilon}{\epsilon_{0}}$ at each point, then the far-zone backscattered field is zero, provided that the incidence direction is parallel to an axis of the body about which a rotation of $90^{\circ}$ leaves the shape of the body, together with its material medium invariant.

Theorem 2: If a plane wave is incident on a body composed of a material such that the total field components satisfy the impedance boundary condition, i.e. $\eta=\eta_{s}$, and if the surface is invariant under a $90^{\circ}$ rotation, the backscattering field is zero if the direction of incidence is along the axis of symmetry, where $\eta_{s}$ are equivalent surface impedance and $\eta$ is the impedance of the material employed in the absorber.

So, both theorems depend on the symmetry of the absorber, which is not easily achieved when it comes to real applications. However, if the condition $\frac{\mu}{\mu_{0}}=\frac{\epsilon}{\epsilon_{0}}$ of the Theorem 1 is obeyed, the surface will present an impedance equals to the free-space. Hence, the reflection coefficient between the absorber and the free-space will be zero. However, this is applied only to specular fields. The contribution of the nonspecular fields to the backscattered fields is null only if the geometrical restrictions were obeyed.

\subsection{2}

\section{Rozanov's Theory}

Rozanov's theory presents a physical bound for electromagnetic-wave absorbers relating the bandwidth and its thickness [43], which shows that the bandwidth of the absorber is limited by its thickness. This theory can be applied for any metal-backed absorber under normal incidence and can be expressed as follows

$$
\left|\int_{0}^{\infty} \ln \right| R(\lambda)|d \lambda| \leq 2 \pi^{2} \sum_{i} \mu_{s, i} d_{i},
$$


where $R$ is the reflection coefficient profile, $\lambda$ is the free-space wavelength, and $\mu_{s, i}$ and $d_{i}$ are the thickness and the static permeability of the $i$ th layer of the slab, respectively. Considering a nonmagnetic absorber, i.e. $\mu_{s}=1$, the thickness limit of an absorber can be rewritten as

$$
d \geq \frac{\left|\int_{0}^{\infty} \ln \right| R(\lambda)|d \lambda|}{2 \pi^{2}}
$$

where $d$ is the total thickness of the nonmagnetic absorber.

Therefore, Equation 3-8 can be used for design purposes and provides the minimum thickness for a given frequency response of the reflection coefficient. Moreover, after some experimental analysis, it was shown that the frequency response of the reflection coefficient, in $\mathrm{dB}$, can be approximated by a piecewise function, that is expressed as

$$
R(f)= \begin{cases}-R_{0}\left(\frac{f-f_{1}}{f_{2}-f_{1}}\right) & f_{1} \leq f \leq f_{2} \\ -R_{0} & f_{2} \leq f \leq f_{3} \\ R_{0}\left(\frac{f-f_{4}}{f_{4}-f_{3}}\right) & f_{3} \leq f \leq f_{4} \\ 0 & \text { otherwise }\end{cases}
$$

where $R_{0}$ is the average level of the reflection coefficient, in $\mathrm{dB}$, between $f_{1}$ and $f_{4}$. Besides that, the above equation must be mapped into the wavelength domain before its use in Equation 3-8.

\section{4}

\section{Metamaterial-based Absorber}

An electromagnetic-wave absorber that uses the concepts of metamaterial is often called metamaterial-based absorber or metamaterial perfect absorber (MPA), and, thus, the theory of metamaterial (Section 2.7) can be applied in the structures seen in Section 3.2. Usually, MPAs present a sub-wavelength unit cell that is arranged periodically in order to tailor the effective permeability and permittivity to provide an impedance matching between the free-space and the MPA, and, therefore, a near unity absorptivity over the frequency band of interest can be achieved.

An MPA can be considered a resonant absorber or, more specifically, a circuit analog absorber. In general terms, a single layer MPA consists of three layers. The top layer is a metallic geometric pattern arranged periodically and the bottom layer is a continuous metallic plate, which plays the role of a ground plane. Besides that, these two layers are separated by a dielectric material, in which its thickness is a fraction of the operational wavelength. The main goal of an MPA is to minimize the reflectance and transmittance to achieve a near 
unity absorption, as can be inferred from Equation 3-1. The transmittance is null in the presence of a continuous ground plane and the reflectance can be reduced by choosing properly the design of the geometric pattern and the dielectric layer.

The choice of a proper dielectric substrate is critical to the performance of an MPA. The dielectric should provide space enough to the incident electromagnetic wave stay within the structure and be absorbed. Hence, the thickness and permittivity of the material must be carefully chosen. Moreover, it is important to point out that the absorption within the substrate occurs due to the imaginary part of the permittivity and permeability. Although the values of these quantities are usually small, it is enough to provide a high absorption.

As can be seen in Subsection 3.2.3, the presence of metallic components in the MPA can provide an ohmic loss. Still, this ohmic loss is usually irrelevant when comparing to the dielectric loss. The main role of the metallic pattern is, along with the dielectric and ground plane, to provide an effective medium that matches with free-space and, thus, minimizing the reflection. So, the incident electromagnetic wave does not experiment reflection on the surface of the absorber and its energy is dissipated within the substrate. Beside that, for the MPA be interpreted as an effective medium, the size of its unit cell must be a small fraction of the wavelength.

The first experimental verification of an MPA was performed by Landy et al. in 2008 [9]. The proposed MPA presented two metallic layers and one dielectric layer. Besides that, in a single unit cell, as it is shown in Figure 3.6(a), the top and bottom metal layers consist of an electric ring resonator (ERR) and a cut wire, respectively. It was verified that the proposed MPA presented both electric and magnetic response. The electric response is provided by the ERR along with the cut wire coupling to the incident electric field at a specific frequency. On the other hand, the magnetic response is achieved due to the antiparallel directions of the surface current densities on the top and bottom layers. This structure presented an absorptivity of $\approx 99 \%$ at $11.48 \mathrm{GHz}$, as can be seen in Figure 3.6(b).

Since the first successful experimental realization of an MPA, the interest in this kind of structure has been growing exponentially. Different types of MPA have been proposed from $\mathrm{GHz}$ to the $\mathrm{THz}$ realm. Furthermore, the advent of metamaterials in electromagnetic-wave absorbers has been proving very versatile, and, therefore, a great number of MPAs has been presented in the literature with broadband, wide-angle stability, polarization insensitivity, small size, multiband, small cost, and so on. 


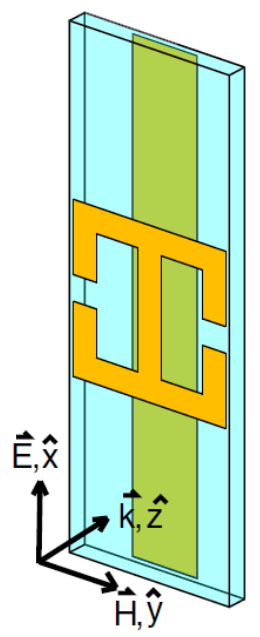

(a)

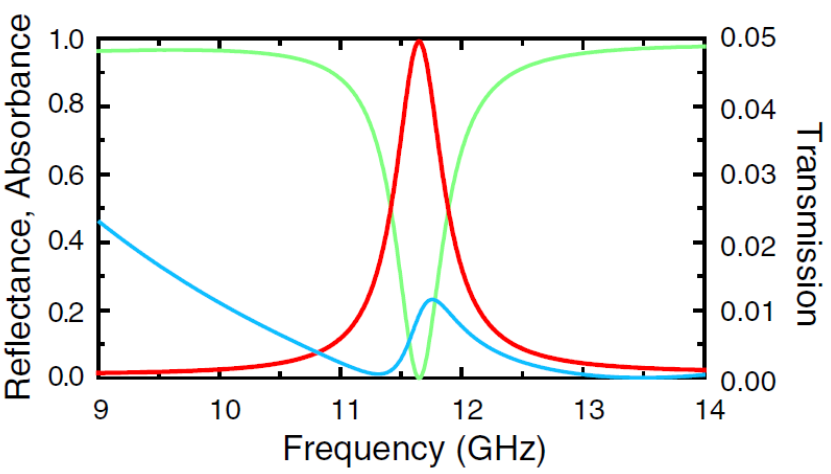

(b)

Figure 3.6: Unit cell of the MPA proposed by Landy (a) and the plot of absorbance (b) [9].

\section{5}

\section{Interpretation and Characterization of Absorbers}

This Section presents means to interpret and to characterize electromagnetic-wave absorbers. Usually, equivalent circuits are used for this purpose as well as the interpretation of the absorber as a stratified medium. Therefore, these two methods will be presented hereafter in this Section.

\subsection{1}

\section{Equivalent Circuit}

One of the most popular and efficient ways to understand the principles of operation of an electromagnetic-wave absorber is defining and grasping its equivalent circuit. For this purpose, the transmission line theory (TLT) is applied to provide means to comprehend the structure at issue. Also, LC circuits have been proven to be effective to characterize the behavior of resonant absorbers [44]. The number of lumped elements employed in these equivalent circuits depends on the number of resonances presented by the absorber. For example, a single resonant absorber presents only one series RLC in parallel with the grounded dielectric. A generic equivalent circuit for a metal-backed absorber is shown in Figure 3.7, where $Z_{d}$ is the impedance of the grounded substrate, and $\epsilon$ and $\mu$ are permittivity and permeability of the substrate, respectively.

Using the TLT is possible to describe the impedance of the grounded dielectric, for both TE and TM polarization, as 


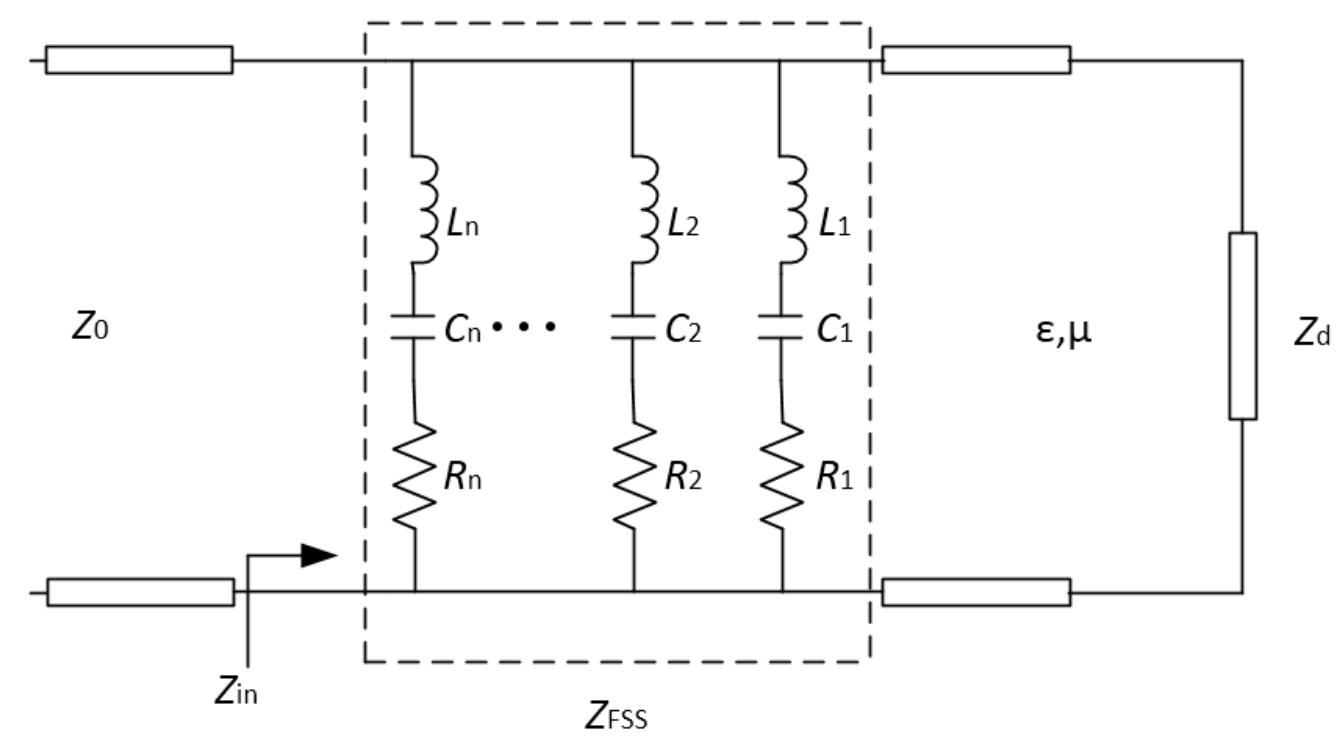

Figure 3.7: Equivalent circuit of a resonant absorber.

$$
\begin{aligned}
& Z_{d}^{\mathrm{TE}}=j \frac{\omega \mu}{\beta} \tan \left(\beta_{\mathrm{TE}} d\right), \\
& Z_{d}^{\mathrm{TM}}=j \frac{\beta}{\omega \epsilon} \tan \left(\beta_{\mathrm{TM}} d\right),
\end{aligned}
$$

where $\beta_{\mathrm{TM}}=\beta_{\mathrm{TE}}=\sqrt{k^{2}-k_{t}^{2}}$ is the propagation constant along the normal unitary vector of the absorber, $k_{t}=k_{0} \sin \theta$ is the transverse wavenumber, and $\theta$ is the angle of incidence of the impinging wave. Besides that, it is important to note that when the thickness of the grounded dielectric is smaller than the quarter of the wavelength, the impedance of the grounded dielectric becomes inductive [40].

The input impedance of the equivalent circuit is given by the parallel connection between the impedance of the grounded dielectric $Z_{d}$ and the impedance of the RLC circuits. So, the reflection coefficient of the equivalent circuit can be written as

$$
\left|\Gamma^{\mathrm{TE}, \mathrm{TM}}\right|=\sqrt{\frac{\left(\operatorname{Re}\left\{Z_{\text {in }}\right\}-Z_{0}^{\mathrm{TE}, \mathrm{TM}}\right)^{2}+\left(\operatorname{Im}\left\{Z_{\text {in }}\right\}\right)^{2}}{\left(\operatorname{Re}\left\{Z_{\text {in }}\right\}+Z_{0}^{\mathrm{TE}, \mathrm{TM}}\right)^{2}+\left(\operatorname{Im}\left\{Z_{\text {in }}\right\}\right)^{2}}},
$$

where $Z_{0}^{\mathrm{TE}, \mathrm{TM}}$ is the characteristic impedance of free-space for TE or TM polarization, and are written, respectively, as follow

$$
\begin{gathered}
Z_{0}^{\mathrm{TE}}=\frac{Z_{0}}{\cos \theta}, \\
Z_{0}^{\mathrm{TM}}=Z_{0} \cos \theta,
\end{gathered}
$$

where $Z_{0}$ is the characteristic impedance of free-space and its value is approximately $377 \Omega$. 
The optimal values of the lumped elements must provide means to the maximum absorption, which occurs when the following conditions are respected

$$
\begin{gathered}
\operatorname{Re}\left\{Z_{\text {in }}\right\}=Z_{0}^{\mathrm{TE}, \mathrm{TM}}, \\
\operatorname{Im}\left\{Z_{\text {in }}\right\}=0 .
\end{gathered}
$$

To find the optimal values of the lumped elements of the equivalent circuit, a reference of the input impedance $Z_{\text {in }}$ can be calculated using the reflection coefficient $\Gamma$ of the absorber from the electromagnetic simulation, and, thus, $Z_{\text {in }}$ can be easily determined. Hence, the input impedance can be written as

$$
Z_{\text {in }}=Z_{0} \frac{1+\Gamma}{1-\Gamma}
$$

This value of $Z_{\text {in }}$ is used as a reference to the extraction of the lumped elements of the equivalent circuit.

To provide a better understanding of how the equivalent circuit can provide an efficient way to interpret the mechanism of absorption, a singleresonant absorber is considered from now on and its equivalent circuit is presented in Figure 3.8.

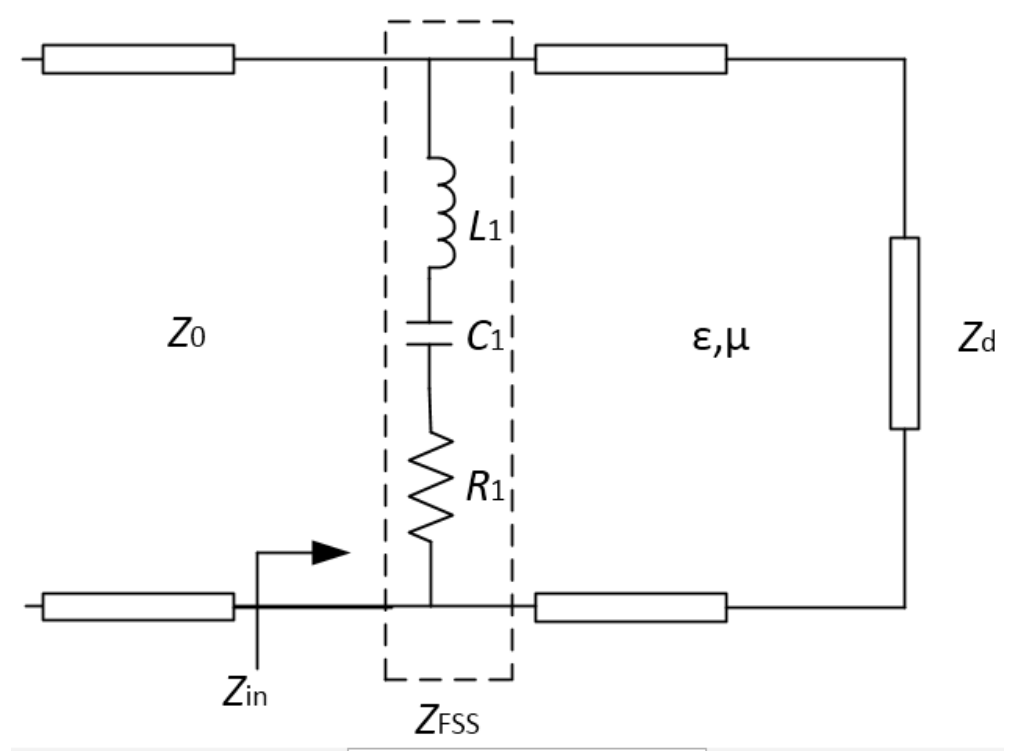

Figure 3.8: Equivalent circuit of a single-resonant absorber.

The impedance of the lossy FSS can be expressed as

$$
Z_{\mathrm{FSS}}=R-j\left(\frac{1-\omega^{2} L C}{\omega C}\right) .
$$

Furthermore, $Z_{\text {in }}$ is equal to the parallel connection between $Z_{\mathrm{FSS}}$ and $Z_{\mathrm{d}}$, which is written as 


$$
Z_{\text {in }}=\frac{Z_{\mathrm{d}} Z_{\mathrm{FSS}}}{Z_{\mathrm{d}}+Z_{\mathrm{FSS}}}
$$

When the inductive impedance of the grounded substrate $Z_{\mathrm{d}}$ is equal to the imaginary part of $Z_{\mathrm{FSS}}$, the parallel circuit resonates and the impedance $Z_{\text {in }}$ is purely real for thin substrates [45], which can be represented as

$$
\operatorname{Re}\left\{Z_{\text {in }}\right\}=\frac{\left(Z_{d}^{\mathrm{TE}, \mathrm{TM}}\right)^{2}}{R} .
$$

The absorption of the impinging wave occurs when the real part of the input impedance $Z_{\text {in }}$ matches to the free-space impedance, and, thus, an optimum value of resistance $R_{\text {opt }}$ of the RLC circuit can be written as follows

$$
R_{\mathrm{opt}}=\frac{\left(Z_{d}^{\mathrm{TE}, \mathrm{TM}}\right)^{2}}{Z_{0}^{\mathrm{TE}, \mathrm{TM}}} .
$$

The value of lumped resistance $R$ can be related to the surface resistance of the FSS $R_{s}$ as well. This relation means that the optimal surface resistance of the FSS must be equal to $R_{\text {opt }}$. Besides that, the shape of the unit cell, the resistance of the sheet employed, the thickness and the dielectric constant of the substrate are important parameters in which the surface resistance depends on. A good estimative of the lumped resistance taking into account the surface resistance of the FSS is given by

$$
R \approx R_{s} \frac{D^{2}}{A}
$$

where $D$ is the periodicity of the unit cell and $A$ is the area of the lossy element parallel to the impinging electric field within a unit cell.

Some classical configurations of absorbers present straightforward equations to calculate the values of the lumped elements in terms of the geometric parameters. For instance, the Jerusalem cross and the patch present welldefined equivalent circuits and equations [46], [47].

\subsection{2}

\section{Reflection and Transmission in a Stratified Medium}

The reflection and transmission method provides a way to understand the process of absorption in a structure with single or multiples layers. This method uses the amplitudes and phases of the reflection and transmission coefficients to show that a destructive interference can occur within the absorber, and, therefore, providing a high level of absorption. The overall reflection and transmission are results of the multiple reflections and transmissions at the multiples interfaces. Additionally, this Section presents the development for a TE wave for the sake of simplicity, the results for TM can be found in [48], [49]. 
Considering a stratified medium with $n+1$ regions, as it is represented in Figure 3.9, for an incident TE waves, the electric and magnetic fields in region $l$, taking into account that all field vectors are dependent of $z$ and $x$ and independent of $y$, can be expressed as

$$
\begin{gathered}
E_{l y}=\left(A_{l} e^{i k_{l z} z}+B_{l} e^{-i k_{l z} z}\right) e^{i k_{x} x}, \\
H_{l x}=-\frac{k_{l z}}{\omega \mu_{l}}\left(A_{l} e^{i k_{l z} z}-B_{l} e^{-i k_{l z} z}\right) e^{i k_{x} x}, \\
H_{l z}=\frac{k_{x}}{\omega \mu_{l}}\left(A_{l} e^{i k_{l z} z}+B_{l} e^{-i k_{l z} z}\right) e^{i k_{x} x},
\end{gathered}
$$

where $z=-d_{0},-d_{1}, \ldots,-d_{n}$ are the boundaries of each layer; $k_{l z}$ and $k_{x}$ are the components of the incident wave vector, $\epsilon_{l}$ and $\mu_{l}$ are the permeability and permittivity of the $l$ th region, respectively. In addition, $A_{l}$ is the amplitude of all wave components that have a propagating velocity component along $+z$ direction and $B_{l}$, along $-z$ direction.

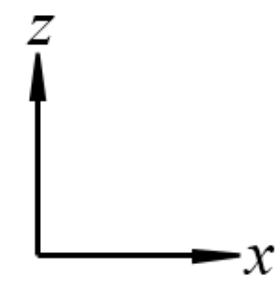

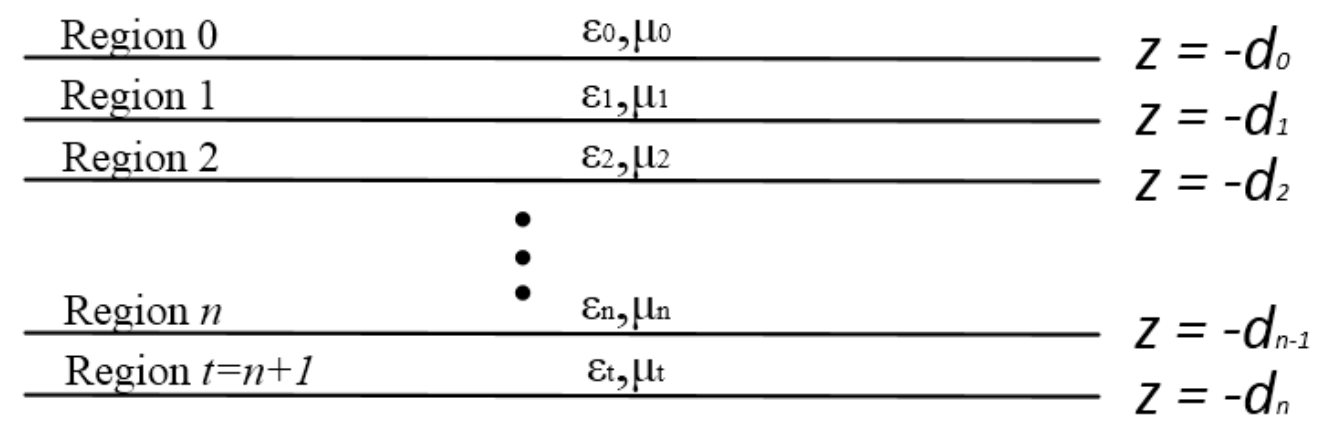

Figure 3.9: Stratified medium.

The field vectors $E_{l y}, H_{l x}$ and $H_{l z}$ satisfy the Helmholtz equation. Besides that, using Maxwell's equation and the field vector $E_{l y}$, the following dispersion relation can be found

$$
k_{l z}^{2}+k_{x}^{2}=\omega^{2} \mu_{l} \epsilon_{l} .
$$

In region 0 , the wave amplitudes $A_{0}$ and $B_{0}$ can be written as

$$
A_{0}=\Gamma E_{0}
$$




$$
B_{0}=E_{0}
$$

For region $t$,

$$
\begin{gathered}
A_{t}=0, \\
B_{t}=\mathrm{T} E_{0},
\end{gathered}
$$

where $E_{0}$ is the amplitude of the incident electromagnetic wave, $\Gamma$ is the reflection coefficient, and $\mathrm{T}$ is the transmission coefficient.

Applying the boundary conditions presented in Section 2.3 at the interface $z=-d_{l}$, the following relations can be obtained

$$
\begin{gathered}
A_{l} e^{-i k_{l z} d_{l}}+B_{l} e^{i k_{l z} d_{l}}=A_{l+1} e^{-i k_{(l+1) z} d_{l}}+B_{l+1} e^{i k_{(l+1) z} d_{l}}, \\
\frac{k_{l z}}{\mu_{l}}\left(A_{l} e^{-i k_{l z} z}-B_{l} e^{i k_{l z} z}\right)=\frac{k_{(l+1) z}}{\mu_{l+1}}\left(A_{l+1} e^{-i k_{(l+1) z} z}-B_{l+1} e^{i k_{(l+1) z} z}\right) .
\end{gathered}
$$

Although a stratified medium presents multiples reflections and transmissions due to its multiple interfaces, when it comes to absorbers, the overall reflection coefficient in region 0 is enough to understand the mechanism of absorption. The electromagnetic-wave absorbers considered in this work present a ground plane, which provides a transmission coefficient of -1 . Moreover, a simple way to find these coefficients will be described in the following sections.

\subsubsection{1}

\section{Reflection Coefficient}

To find the close-form formula for reflection coefficient $\Gamma$, the Equations 3-30 and 3-31 are solved and rewritten as follow

$$
\begin{aligned}
& A_{l} e^{-i k_{l z} d_{l}}=\frac{1}{2}\left(1+p_{l(l+1)}\right)\left\{A_{l+1} e^{-i k_{(l+1) z} d_{l}}+R_{l(l+1)} B_{l+1} e^{i k_{(l+1) z} d_{l}}\right\}, \\
& B_{l} e^{i k_{l z} d_{l}}=\frac{1}{2}\left(1+p_{l(l+1)}\right)\left\{R_{l(l+1)} A_{l+1} e^{-i k_{(l+1) z} d_{l}}+B_{l+1} e^{i k_{(l+1) z} d_{l}}\right\},
\end{aligned}
$$

where $p_{l(l+1)}$, for a TE wave, is expressed as

$$
p_{l(l+1)}=\frac{\mu_{l} k_{(l+1) z}}{\mu_{l+1} k_{l z}}
$$

and the reflection coefficient in region $l$ provided by the interface between regions $l$ and $l+1$ can be written as

$$
R_{l(l+1)}=\frac{1-p_{l(l+1)}}{1+p_{l(l+1)}}
$$


It is possible to note in Equation 3-34 that $p_{l(l+1)}=\frac{1}{p_{(l+1) l}}$, which provides the following expression

$$
R_{(l+1) l}=-R_{l(l+1)} .
$$

The amplitudes $A_{l}$ and $B_{l}$, depending on the referential, can be interpreted as the amplitude of the reflected and transmitted waves. So, to find the reflection coefficient of the region $l$ is important to solve the ratio $A_{l} / B_{l}$ that is

$$
\begin{aligned}
\frac{A_{l}}{B_{l}}=\frac{e^{i k_{l z} d_{l}}}{R_{l(l+1)}}+ & \frac{\left[1-\left(1 / R_{l(l+1)}^{2}\right)\right] e^{i 2\left(k_{(l+1) z}+k_{l z}\right) d_{l}}}{\left[1 / R_{l(l+1)}\right] e^{i 2 k_{(l+1) z} d_{l}}+\left(A_{l+1} / B_{l+1}\right)} \\
& =\frac{e^{i k_{l z} d_{l}}}{R_{l(l+1)}}+\frac{\left[1-\left(1 / R_{l(l+1)}^{2}\right)\right] e^{i 2\left(k_{(l+1) z}+k_{l z}\right) d_{l}}}{\left[1 / R_{l(l+1)}\right] e^{i 2 k_{(l+1) z} d_{l}}}+\frac{A_{l+1}}{B_{l+1}} .
\end{aligned}
$$

The reflection coefficient of the stratified medium, $\Gamma$, is obtained solving the ratio $A_{0} / B_{0}$ and can be expressed as a continued fraction as

$$
\begin{aligned}
& \Gamma=\frac{A_{0}}{B_{0}}=\frac{e^{i 2 k_{z} d_{0}}}{R_{01}}+\frac{\left[1-\left(1 / R_{01}^{2}\right)\right] e^{i 2\left(k_{1 z}+k_{z}\right) d_{0}}}{\left(1 / R_{01}\right) e^{i 2 k_{1 z} d_{0}}}+\frac{e^{i 2 k_{z} d_{1}}}{R_{12}}+\frac{\left[1-\left(1 / R_{12}^{2}\right)\right] e^{i 2\left(k_{2 z}+k_{1 z}\right) d_{1}}}{\left(1 / R_{12}\right) e^{i 2 k_{2 z} d_{1}}} \\
& +\cdots+\frac{e^{i 2 k_{(n-1) z} d_{n-1}}}{R_{(n-1) n}}+\frac{\left[1-\left(1 / R_{(n-1) n}^{2}\right)\right] e^{i 2\left(k_{n z}+k_{(n-1) z}\right) d_{n-1}}}{\left(1 / R_{(n-1) n}\right) e^{i 2 k_{n z} d_{n-1}}}+R_{n t} e^{i 2 k_{n z} d_{n}} .
\end{aligned}
$$

The closed-form solution can be quite complicated to solve depending on the number of layers, so an alternative solution is to consider the stratified medium as a homogeneous dielectric film, this approach is presented hereafter.

\subsubsection{2}

\section{Reflection and Transmission in a Homogeneous Dielectric Film}

The present method considers a multilayer structure as a single-layer homogeneous film and provides a straightforward way of understanding how and why the absorption occurs. Besides that, it is assumed that all the media are nonmagnetic, i.e. $\mu_{r}=1$, and Figure 3.10 illustrates the problem that considers three media, in which the thickness of the homogeneous dielectric film is $h$.

The Fresnel coefficients at the two interfaces can be written as

$$
r_{12}=\frac{n_{1} \cos \theta_{1}-n_{2} \cos \theta_{2}}{n_{1} \cos \theta_{1}+n_{2} \cos \theta_{2}}
$$




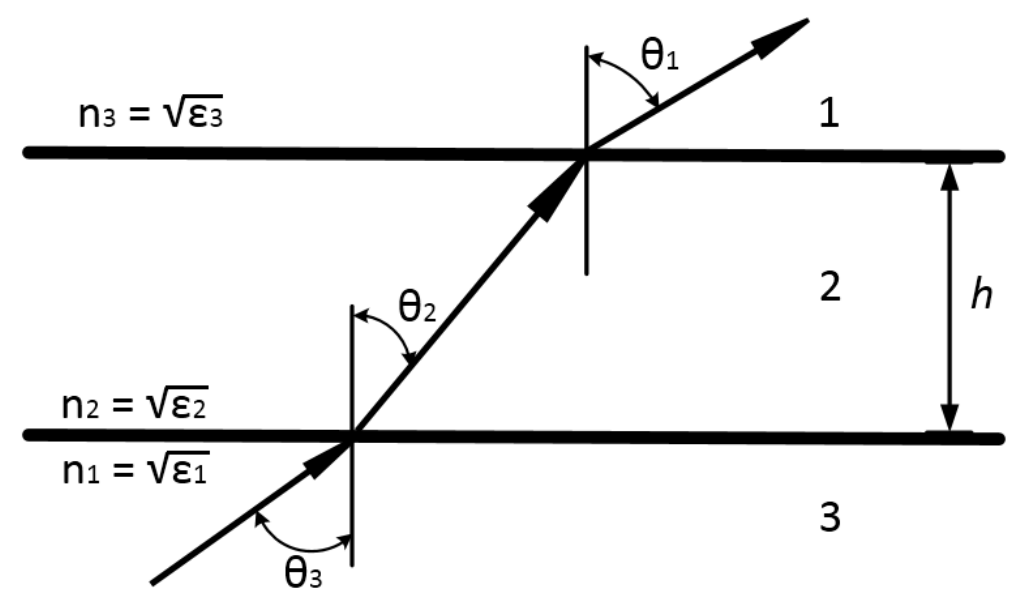

Figure 3.10: Homogeneous dielectric film.

$$
\begin{aligned}
t_{12} & =\frac{2 n_{1} \cos \theta_{1}}{n_{1} \cos \theta_{1}+n_{2} \cos \theta_{2}}, \\
r_{23} & =\frac{n_{2} \cos \theta_{2}-n_{3} \cos \theta_{3}}{n_{2} \cos \theta_{2}+n_{3} \cos \theta_{3}}, \\
t_{23} & =\frac{2 n_{2} \cos \theta_{2}}{n_{2} \cos \theta_{2}+n_{3} \cos \theta_{3}},
\end{aligned}
$$

where $n_{1}, n_{2}$ and $n_{3}$ are the refraction index of region 1,2 and 3 , respectively. Furthermore, considering that the overall reflection and transmission coefficients are the superposition of the multiples reflections and transmission at the two interfaces, the overall reflection and transmission coefficients of the homogeneous dielectric film can be written as

$$
\begin{aligned}
& r=\frac{r_{12}+r_{23} e^{2 i \beta}}{1+r_{12} r_{23} e^{2 i \beta}}, \\
& t=\frac{t_{12} t_{23} e^{2 i \beta}}{1+r_{12} r_{23} e^{2 i \beta}},
\end{aligned}
$$

where

$$
\beta=\frac{2 \pi}{\lambda_{0}} n_{2} h \cos \theta_{2}
$$

So, the reflectance $R$ and the transmittance $T$ can be expressed, respectively, as follow

$$
\begin{gathered}
R=|r|^{2}=\frac{r_{12}^{2}+r_{23}^{2}+2 r_{12} r_{23} \cos 2 \beta}{1+r_{12}^{2} r_{23}^{2}+2 r_{12} r_{23} \cos 2 \beta}, \\
T=\frac{p_{3}}{p_{1}}|t|^{2}=\frac{n_{3} \cos \theta_{3}}{n_{1} \cos \theta_{1}} \frac{t_{12}^{2} t_{23}^{2}}{1+r_{12}^{2} r_{23}^{2}+2 r_{12} r_{23} \cos 2 \beta} .
\end{gathered}
$$

Besides that, the software and algorithms of full-wave analysis deal with the scattering parameters. Hence, it is more intuitive to describe the overall reflection, $S_{11_{\text {тотаL }}}$, of an absorber with a different notation [50], which is expressed as 


$$
\begin{gathered}
S_{11_{\text {тота }}}=S_{11}+S_{12} e^{-j \beta} e^{-j \pi} e^{-j \beta} S_{21}+S_{12} e^{-j \beta} e^{-j \pi} e^{-j \beta}\left(S_{22} e^{-j \beta} e^{-j \pi} e^{-j \beta}\right)^{1} S_{21}+ \\
+S_{12} e^{-j \beta} e^{-j \pi} e^{-j \beta}\left(S_{22} e^{-j \beta} e^{-j \pi} e^{-j \beta}\right)^{2} S_{21}+\ldots \\
=S_{11}+S_{12} e^{-j(2 \beta+\pi)} S_{21} \sum_{n=0}^{\infty}\left(S_{22} e^{-j(2 \beta+\pi)}\right)^{n} \\
=S_{11}+\frac{S_{12} e^{-j \beta} e^{-j \pi} e^{-j \beta} S_{21}}{1-S_{22} e^{-j \beta} e^{-j \pi} e^{-j \beta}} \\
=\left|S_{11}\right| e^{j \theta_{11}}+\frac{\left|S_{12}\right|\left|S_{21}\right| e^{j\left(\theta_{12}+\theta_{21}-2 \beta-\pi\right)}}{1-\left|S_{22}\right| e^{j\left(\theta_{22}-2 \beta-\pi\right)}} .
\end{gathered}
$$

This method provides an intuitive, efficient and straightforward way to describe electromagnetic absorber. This is because it provides a methodology that can be easily integrated with electromagnetic simulators. 


\section{4 \\ Design and Characterization of Metamaterial Absorbers}

This Chapter introduces a novel design for broadband metamaterial absorbers. Moreover, results considering different parameters and materials are presented in order to provide an understanding of the mechanism of absorption in the structure at issue and its applicability in a variety of study fields.

To provide an extra tool to the comprehension of broadband absorbers, an equivalent circuit is proposed as well as an original algorithm based on the least-square method to provide a way to extract the lumped elements of the circuit.

The employment of the proposed absorber in antennas and array is investigated. Low-RCS antennas are introduced and the results are discussed. Also, a structure based on the proposed metamaterial is considered to decrease the mutual coupling in microstrip antenna arrays.

In order to demonstrate the features of the proposed absorber, experimental measurements are presented, and issues regarding the manufactured sample and the measurement setup are detailed. Furthermore, the experimental results are compared with the simulated ones and discussed.

\section{1}

\section{Parametric Approach}

The main goal of this work is to develop a broadband, low-profile and low-cost metamaterial absorber in the $\mathrm{X}$ and $\mathrm{Ku}$ bands, once these frequency ranges are very useful for radars, satellites, broadcasting, and so on. For this purpose, a multi-resonant absorber is initially considered using cheap and easy to find dielectric substrates such as FR-4 and RT/DUROID. Furthermore, the software Ansys HFSS (High-Frequency Structure Simulator) is used during the design and simulation of the structures, in which an infinite array is expanded from a unit cell by using master/slave boundaries and Floquet ports, saving implementation and simulation time.

At first, the geometric pattern used by the metamaterial is based on square spirals, as can be seen in Figure 4.1. This is because each part of the spiral resonates at different frequencies, and, thus, creating multiple peaks of absorption. Furthermore, as most of the broadband absorbers are multi- 
resonant structure, the choice of the square spiral sounds like a reasonable idea.

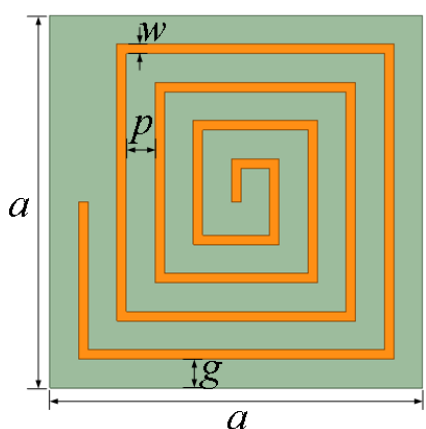

(a)

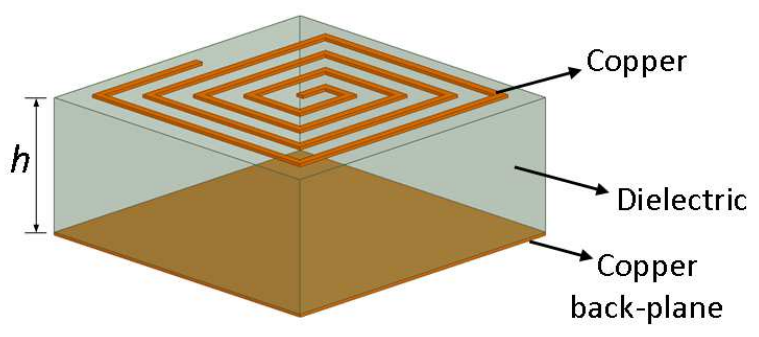

(b)

Figure 4.1: Unit cell of the metamaterial absorber based on square spiral: top (a) and perspective (b) views.

The initial design employs an FR-4 substrate, which has a dielectric constant of 4.4 and a loss tangent of 0.02 . Furthermore, the used metal is copper, which has $5.8 \times 10^{7} \mathrm{~S} / \mathrm{m}$ of conductivity and $35 \mu \mathrm{m}$ of thickness. It is important to note that all the proposed absorbers in this work present a ground plane. This is because the presence of a ground plane provides a null transmittance $T(\omega)$, and, therefore, in Equation 3-1 the only worry is the reflectance $R(\omega)$, once there is no transmission. Moreover, the absence of a ground plane would create an extra problem, which is the interference provided by the environment behind the absorber or the type of material in which the absorber would be placed.

In order to provide an insight of how the square spiral is able to present multiples peaks of resonance, Figure 4.2 shows the curve of absorptivity of an absorber using the geometric pattern at issue, in which its dimensions, in mm, are $a=9.2, w=0.6, p=0.2, g=0.3$, and $h=1.2$. It is possible to observe multiple peaks of absorption above $80 \%$ from 2 to $20 \mathrm{GHz}$. Besides that, this structure is quite promising due to their dimensions. To be more specific, the periodicity $a$ and the thickness $h$ are, in terms of the wavelength $\lambda_{L}$ at the lowest frequency $(4.7 \mathrm{GHz}), 0.1457 \lambda_{L}$ and $0.0188 \lambda_{L}$, respectively. These values show that the dimensions of this absorber are sub-wavelength, which is an essential feature when it comes to nowadays applications.

To understand the mechanism of absorption, the surface current densities are plotted at the frequencies of the three highest peaks of absorption, which are $4.7 \mathrm{GHz}, 10.1 \mathrm{GHz}$, and $15.1 \mathrm{GHz}$, as can be observed in Figure 4.3. It can be noted that at these three resonance frequencies the directions of the surface currents on the top and bottom the absorber are antiparallel, which provides the evidence of a magnetic resonance response, as it was aforementioned in 


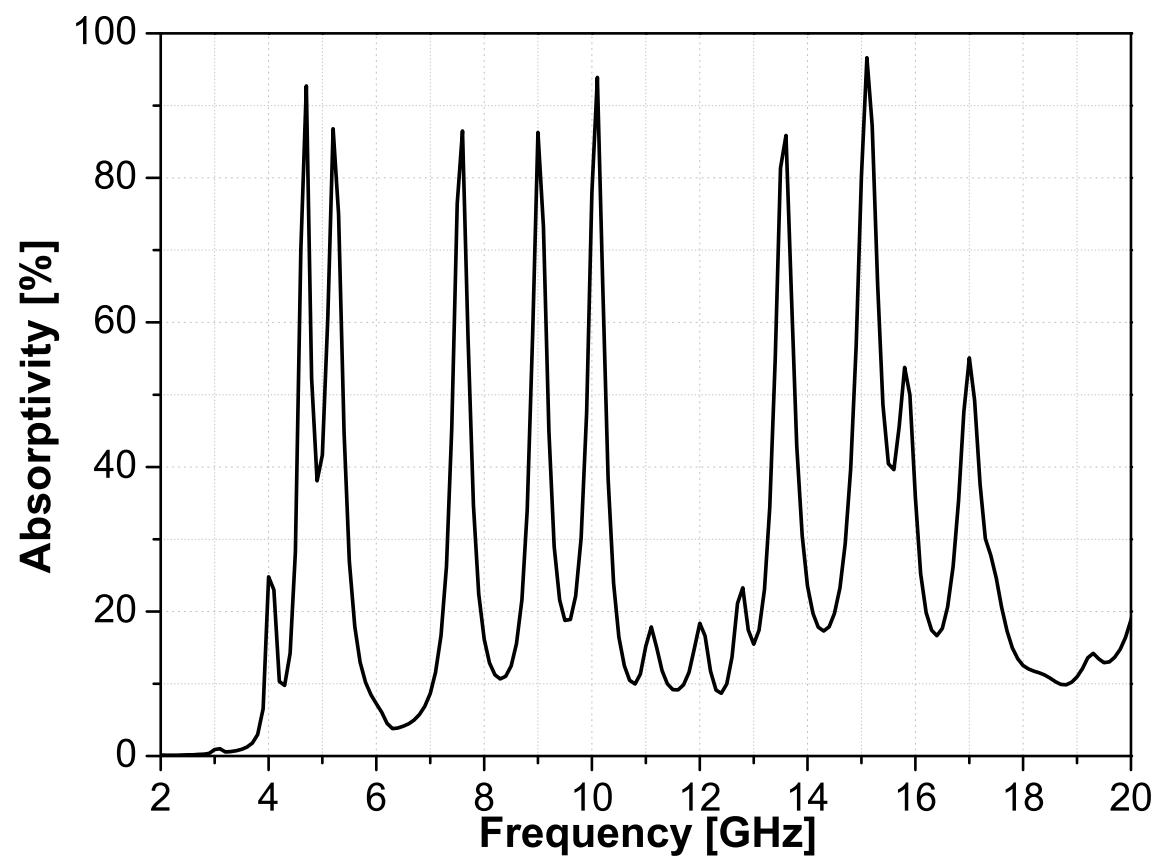

Figure 4.2: Absorptivity of a metamaterial absorber based on square spiral.

Subsection 3.2.3. Furthermore, one can note that different parts of the square spiral are excited at each frequency and with different intensities, which is expected for a multi-resonant structure.

Although the initial absorber presents interesting and promising results with its multiples peaks of resonance, which can find an important role in a variety of applications, the main goal of this work is to develop a broadband absorber in the $\mathrm{X}$ and $\mathrm{Ku}$ bands. So, it is necessary to employ some technique that makes possible to turn the square spiral-based absorber into a broadband one. To fulfill this requirement, cuts are introduced into the spiral in order to provide a redistribution of charges, and, thus, creating more electric and magnetic dipoles, and a broadening in the absorption bandwidth can be achieved [51]. Figure 4.4 shows the proposed metamaterial absorber, in which the gaps are on the same line segment and $\alpha$ is the rotation angle of the line in which the gaps are placed.

It can be observed in Figure 4.3 that the biggest concentrations of surface current density are around the turns of the spiral. Therefore, at first, the gaps were placed exactly at the turns of the spiral with $\alpha=45^{\circ}$, in order to provide an effective redistribution of charges. Moreover, the effect of the variation of $\alpha$ will be discussed further in this work.

Intuitively, the thickness of the substrate is inversely proportional to the central frequency of absorption. In order to prove this statement and to show the design flexibility of the proposed absorber, Figure 4.5 illustrates the curves 


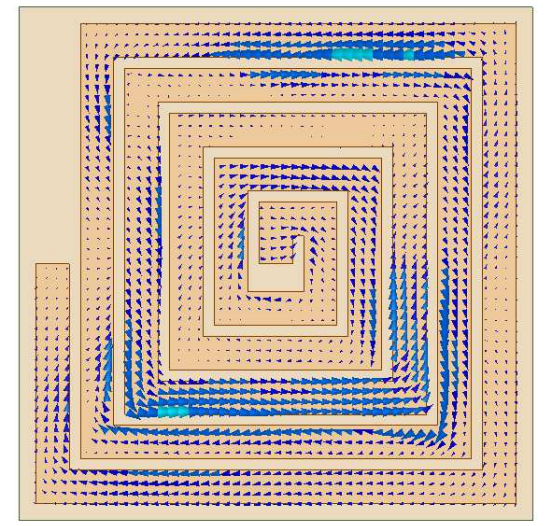

(a)

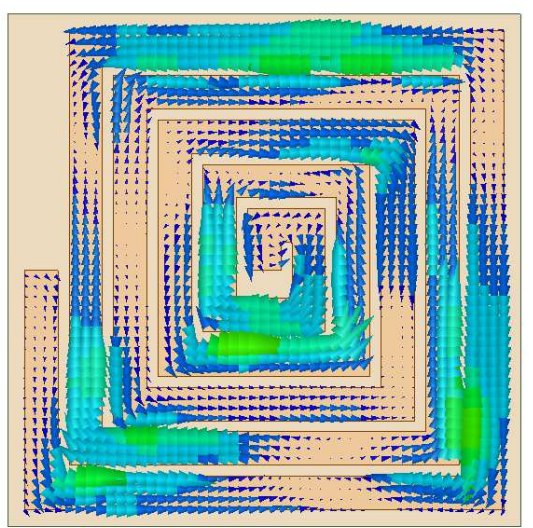

(b)

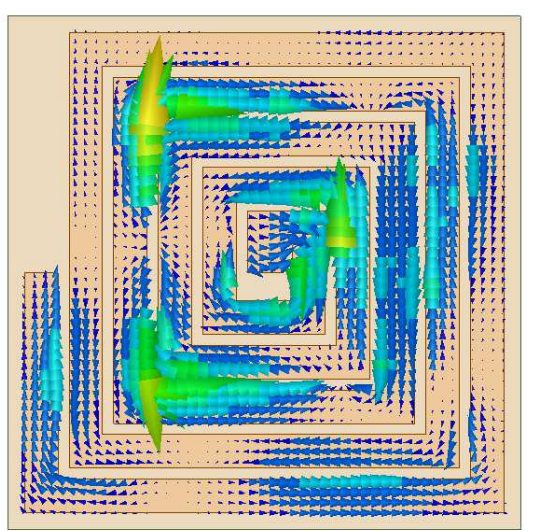

(c)

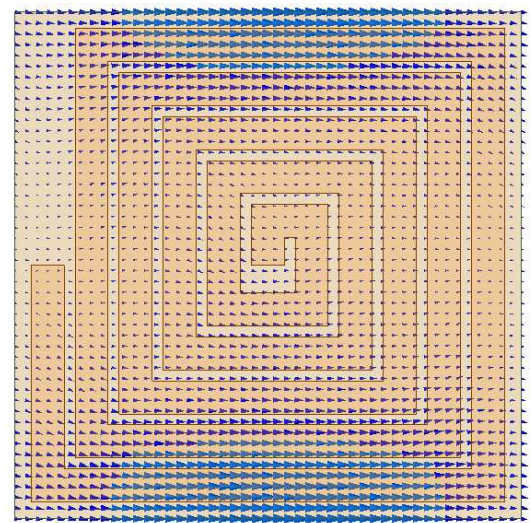

(d)

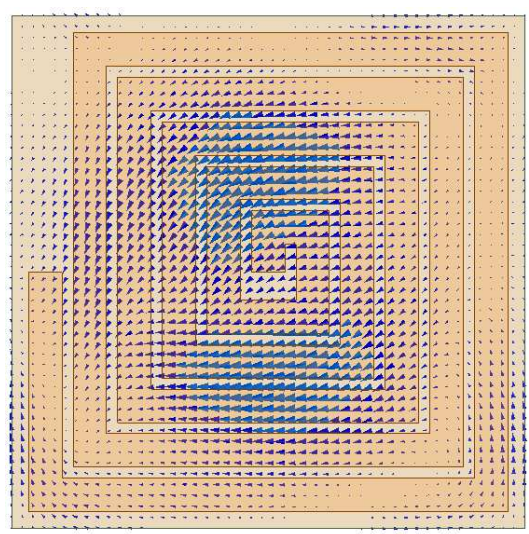

(e)
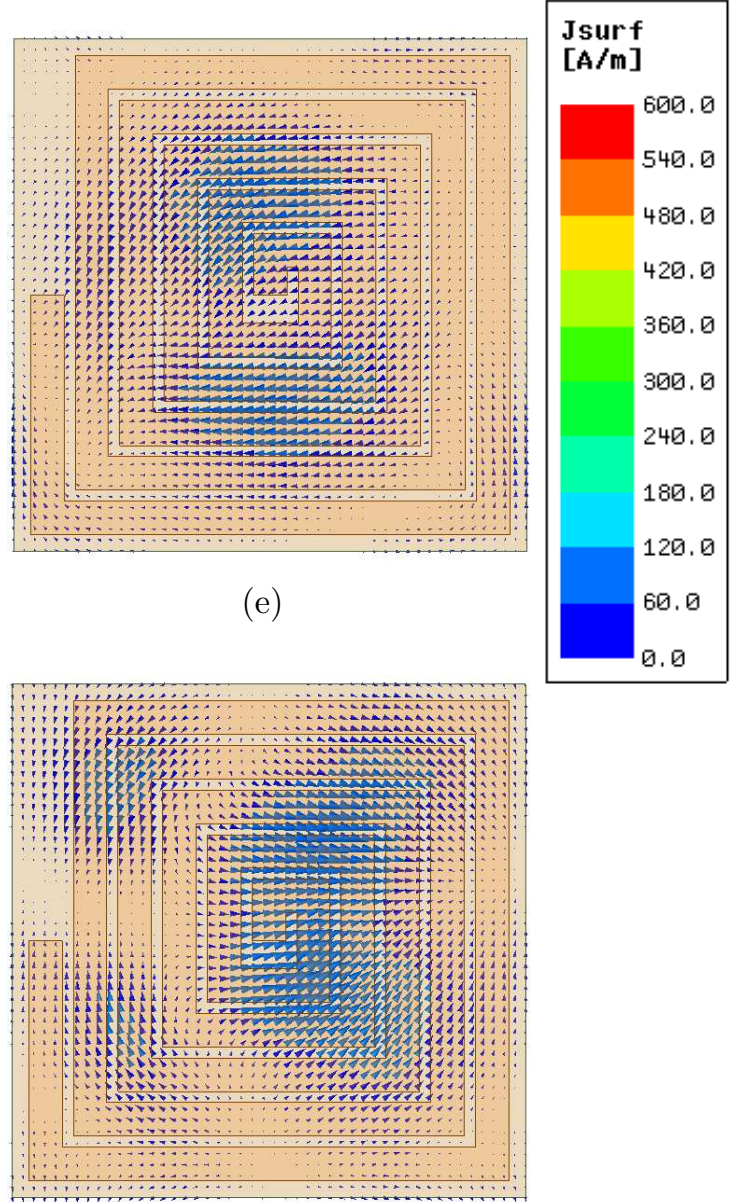

(f)

Figure 4.3: Simulated surface current density on the top at (a) $4.7 \mathrm{GHz}$, (b) $10.1 \mathrm{GHz}$, and (c) $15.1 \mathrm{GHz}$, and on the bottom at (d) $4.7 \mathrm{GHz}$, (e) $10.1 \mathrm{GHz}$, and (f) $15.1 \mathrm{GHz}$.

of absorption considering different dimensions and thickness, and Table 4.1 shows the dimensions of each proposed metamaterial absorber. In addition, the FR-4 substrate is employed in all considered models. It is possible to observe in Figure 4.5 that the frequency band of absorption shifts downward with the 


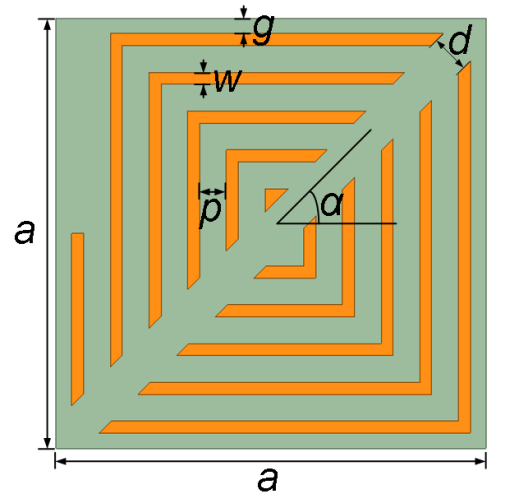

(a)

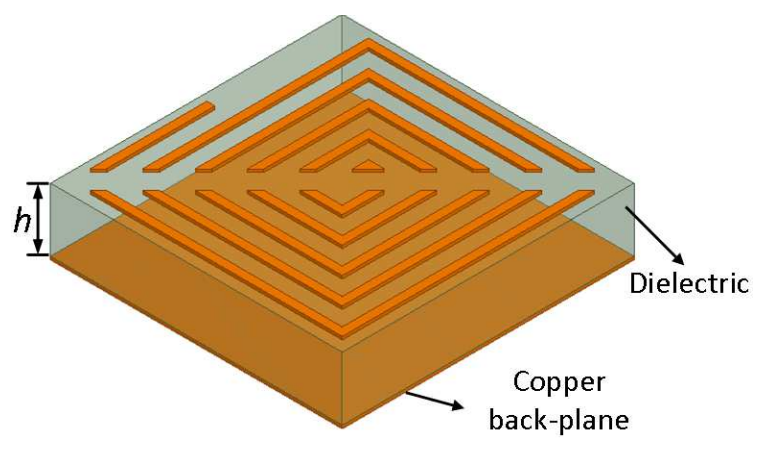

(b)

Figure 4.4: Unit cell of the proposed metamaterial absorber: top (a) and perspective (b) views.

increase of the thickness of the substrate. Furthermore, all considered models present broadband of absorption above $90 \%$, which means that the technique of cutting out parts of the spiral is efficient in turning a multiband absorber into a broadband one. To be more specific, the frequency ranges, in which the absorption is above 90\% for the Model 1, Model 2, Model 3 and Model 4 are, in $\mathrm{GHz}, 11.44$ - 20.00, 10.43 - 15.14, 8.68 - 13.41 and 7.41 - 12.40, respectively.

Table 4.1: Parameters for the models of the proposed metamaterial absorbers.

\begin{tabular}{c|c|c|c|c|c|c|c} 
Absorber & $h[\mathrm{~mm}]$ & $a[\mathrm{~mm}]$ & $w[\mathrm{~mm}]$ & $p[\mathrm{~mm}]$ & $g[\mathrm{~mm}]$ & $d[\mathrm{~mm}]$ & Turns \\
\hline Model 1 & 1.6 & 3.655 & 0.155 & 0.155 & 0.2 & 0.5 & 5 \\
Model 2 & 1.8 & 3.700 & 0.100 & 0.200 & 0.3 & 0.1 & 5 \\
Model 3 & 2.0 & 5.800 & 0.200 & 0.100 & 0.3 & 0.3 & 6 \\
Model 4 & 2.2 & 5.000 & 0.200 & 0.100 & 0.3 & 0.3 & 7
\end{tabular}

It is necessary to choose one of the proposed models to perform further simulations and evaluations, for the sake of organization and simplicity. Among the four proposed models, models 1 and 4 present a great potential, because they cover entirely the $\mathrm{Ku}$ and $\mathrm{X}$ band, respectively. Moreover, both models would equally find applications in their respective frequency band of operation, but the Model 1 has the advantage of its thickness, since the FR-4 substrate with $1.6 \mathrm{~mm}$ of thickness is easier to find and cheaper than those with $2.2 \mathrm{~mm}$ of thickness. Taking this into account, the Model 1 will be used from now on to provide a better understanding of how this metamaterial absorber works.

\subsection{1}

\section{Numerical and Conceptual Analysis}

In this Subsection, a deep investigation of the proposed absorber is provided considering the discussed and raised issues in Chapter 3. Furthermore, 


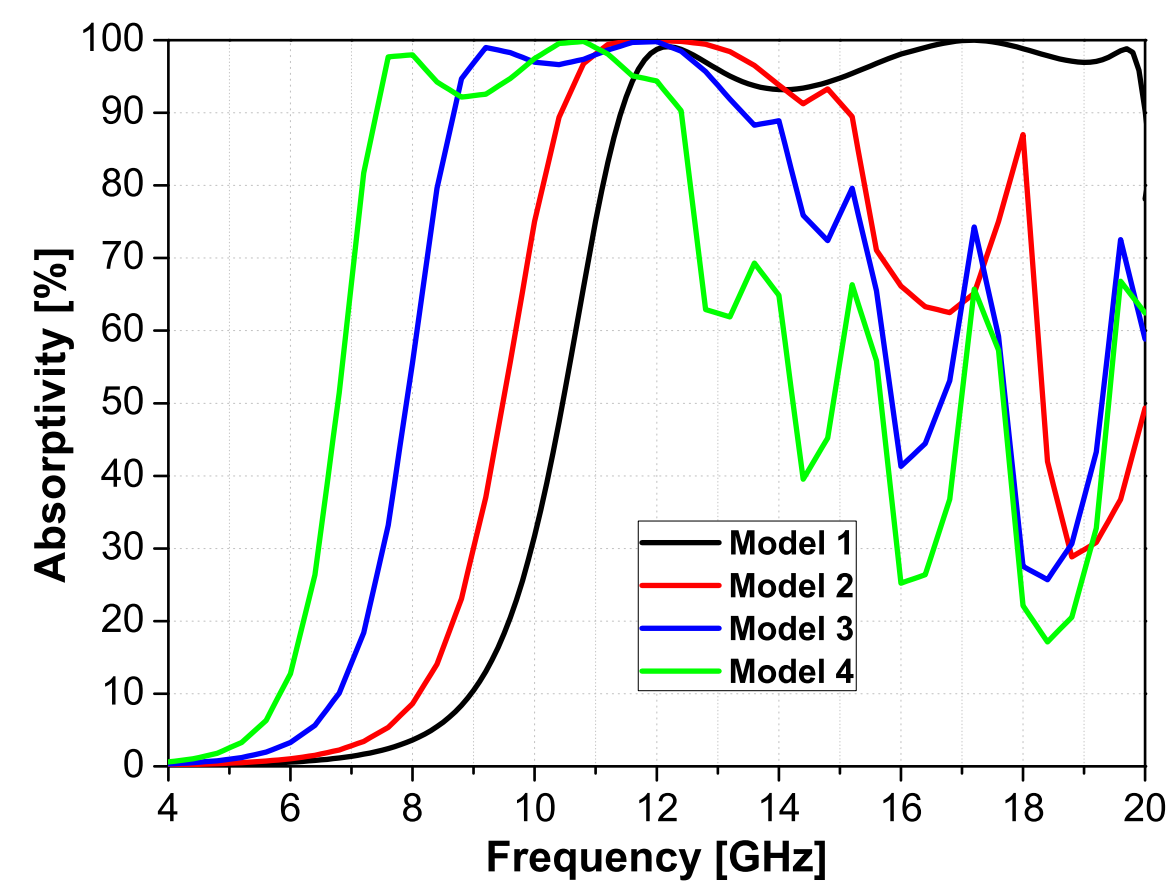

Figure 4.5: Absorptivity of the proposed metamaterial absorber with different dimensions as in Table 4.1.

numerical analyses are carried out to interpret the behavior of the absorber under different circumstances.

To begin with, the most important question is whether the proposed absorber must be considered a metamaterial or a frequency selective surface, according to the definitions presented in Section 2.7. As can be observed in Table 4.1, the periodicity $a$ of the Model 1 is $3.655 \mathrm{~mm}$ or, in terms of the wavelength $\lambda_{L}$ at the lowest frequency $(11.44 \mathrm{GHz}), 0.139 \lambda_{L}$. Therefore, the periodicity of the absorber is much smaller than the wavelength and fulfills the periodicity requirement of a metamaterial. Besides that, the thickness of the absorber is $0.061 \lambda_{L}$, which means that the thickness is much smaller than the wavelength. Taking this into account, this absorber can be considered a metasurface, which is a superficial version of a metamaterial. Moreover, the geometric pattern of the scatter allows the absorber to be classified as a metafilm.

So far in this Chapter, the simulations considered the transverse electric (TE) polarization, and one of the most important and desirable features of an absorber is the polarization insensitivity. Considering this, Figure 4.6 shows the absorptivity of the proposed metamaterial absorber for both TE and transverse magnetic (TM) polarization. The curves are almost the same for both orthogonal polarization at issue, which means that the absorber can be considered to be polarization insensitive. 


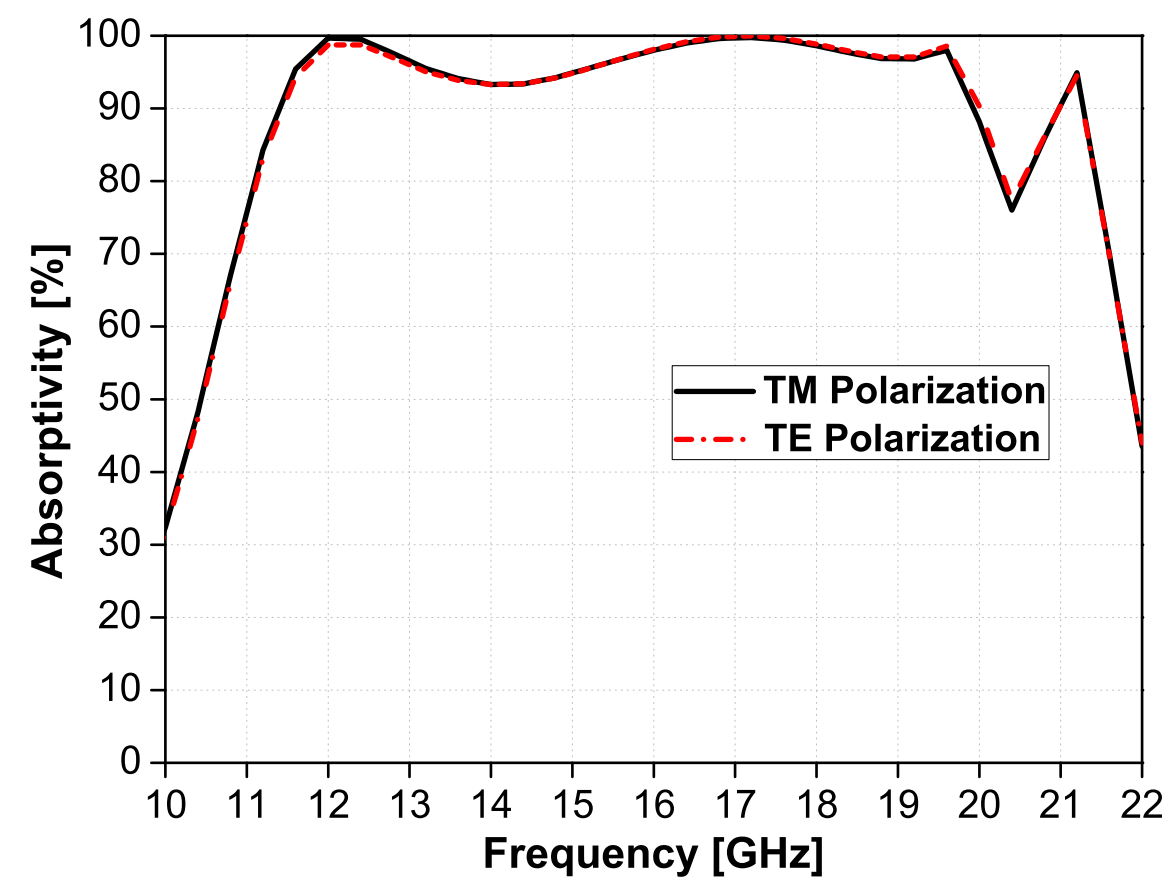

Figure 4.6: Absorptivity of the metamaterial absorber considering both TE and TM polarization.

Besides a great performance of an absorber under normal incidence, it is essential to a good absorber to have wide-angle stability. Therefore, Figures 4.7(a) and (b) show the absorptivity under oblique incidence for the TE as well as the TM polarization, respectively. Considering the absorptivity above $80 \%$ as a reference of a great level of absorption, the proposed metamaterial absorber presents angular stability up to $40^{\circ}$ and $50^{\circ}$ for the TE and TM polarizations, respectively. Hence, the absorber can be considered to have wide angular stability, once the proposed model absorbs above $80 \%$ of the incident energy over a wide range of incidence angles for both orthogonal polarizations, maintaining a wide bandwidth of absorption.

In order to provide an insight into the influence of the position of the gap, Figure 4.8 shows the absorptivity of the proposed absorber based on the model 1 but with $\alpha$ varying from $0^{\circ}$ to $165^{\circ}$ considering steps of $15^{\circ}$. It is possible to note that the highest level and broadest band of absorption occurs when $\alpha$ is equal to $45^{\circ}$ and $135^{\circ}$, in which the gaps are placed on the curves of the spiral, where the maximum concentration of charges would occur in a spiral square without gaps, as can be observed in Figure 4.3. Therefore, the gaps by themselves are not sufficient to transform a multiband absorber into a broadband one, it is necessary to choose carefully where these gaps must be placed.

The presence of the gap in the square spiral presents the possibility of 


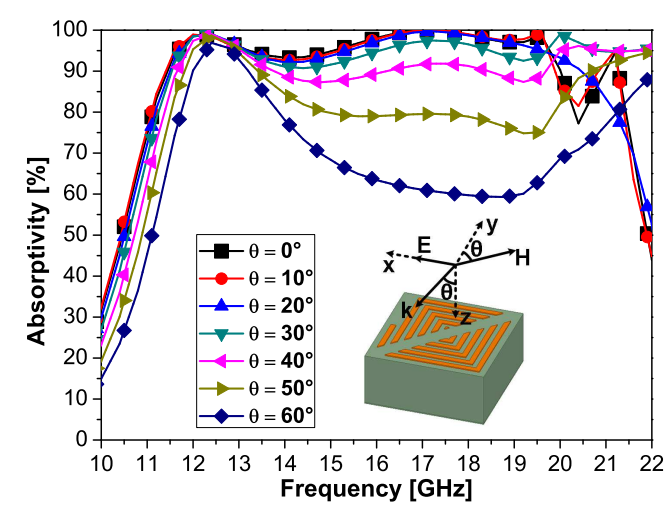

(a)

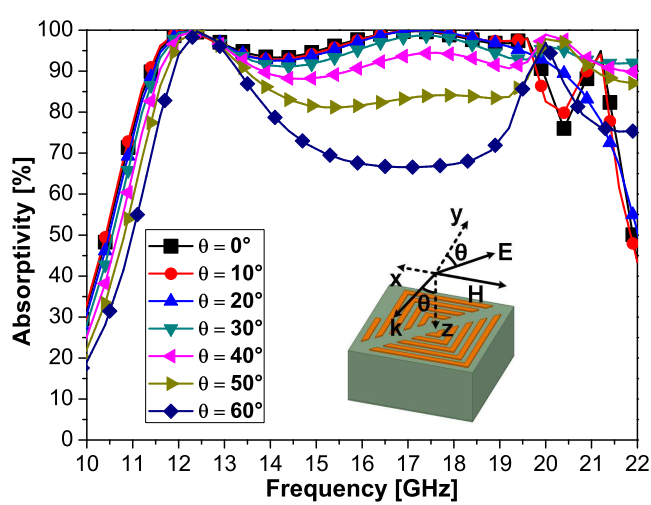

(b)

Figure 4.7: Absorptivity of the metamaterial absorber considering different values of incidence angle $\theta$ for TE (a) and TM (b) polarizations.

a redistribution of charges in the structure, and, thus, electric and magnetic dipoles are created, as it was aforementioned. Taking this into account, the influence of the width of the gap is studied, as can be observed in Figure 4.9. It was analyzed the gap width $d$ varying from 0.1 to $1.0 \mathrm{~mm}$ considering steps of $0.1 \mathrm{~mm}$, with the other dimensions remaining unchanged. One can notice that, for all values of $d$, the structures present broadband of absorption. Furthermore, the value of $d$ is directly proportional to the central frequency of the band of absorption. These results corroborate even more with the highflexibility design of the proposed metamaterial absorber.

As it is discussed in Section 3.3, the Rozanov's theorem demonstrates mathematically that the bandwidth and thickness of the absorber are contradictory parameters for broadband absorbers [43]. For some applications, a broadband absorber that obeys the Rozanov's limit is considered thick. Taking this into account, the Rozanov's theorem is applied to the metamaterial absorber and it is considered the reflection coefficient $S_{11}$ from 5 to $22 \mathrm{GHz}$, which covers the main bandwidth of absorption, and, then, the $S_{11}$ is mapped into the wavelength domain. Using Equation 3-8, the minimum theoretical thickness considering the reflection coefficient over the frequency range at issue is $1.7330 \mathrm{~mm}$, which is $8.31 \%$ bigger than the actual thickness of the proposed absorber, and, thus, showing how thin the metamaterial at issue is.

The performance of the proposed metamaterial absorber is compared with other broadband structures previously presented in the literature, as can be seen in Table 4.2. In this Table, the bandwidth is given considering the absorption above $90 \%$, and the thickness and periodicity are written in terms of the wavelength at the lowest frequency of the band of absorption of each structure. The proposed absorber excels when it comes to the periodicity, which 


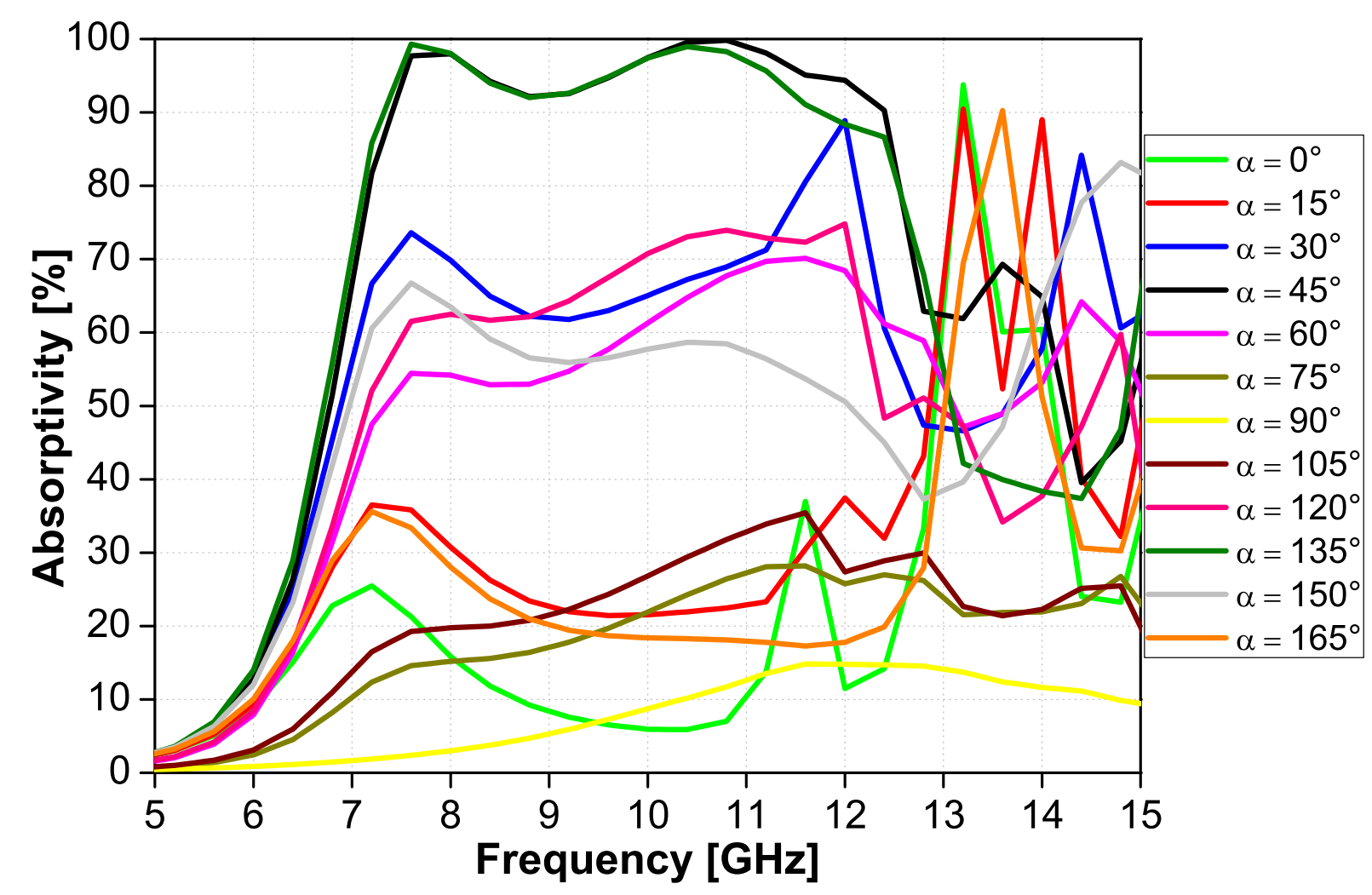

Figure 4.8: Absorptivity of the metamaterial absorber based on Model 4 considering different values of $\alpha$.

is an essential feature for nowadays applications. This is because the smaller the periodicity, the easier the absorber will adapt itself to irregular surfaces. Moreover, the relative bandwidth of the proposed absorber is huge, especially for such an electrically small thickness and considering a single-layer structure. It is important to point out that the structures presented in [52] and [52] have a larger relative bandwidth, but these absorbers employ 8 lumped elements per unit cell, leading to an expensive and complex manufacturing process.

\section{2}

\section{Mechanism of Absorption}

So far, the presented results show that model 1 has all the prerequisites of a good absorber, which are: broadband, polarization insensitivity, low-cost, low-profile, wide angle insensitivity, and easy manufacturing. Despite that, some other results are required in order to provide an insight into how and why the absorption occurs in the proposed model. Moreover, it is possible to observe that the absorber presents three main peaks of absorption at 12.2 $\mathrm{GHz}, 17.1 \mathrm{GHz}$, and $19.7 \mathrm{GHz}$ with absorption levels of $99.11 \%, 99.98 \%$, and $98.83 \%$, respectively. 


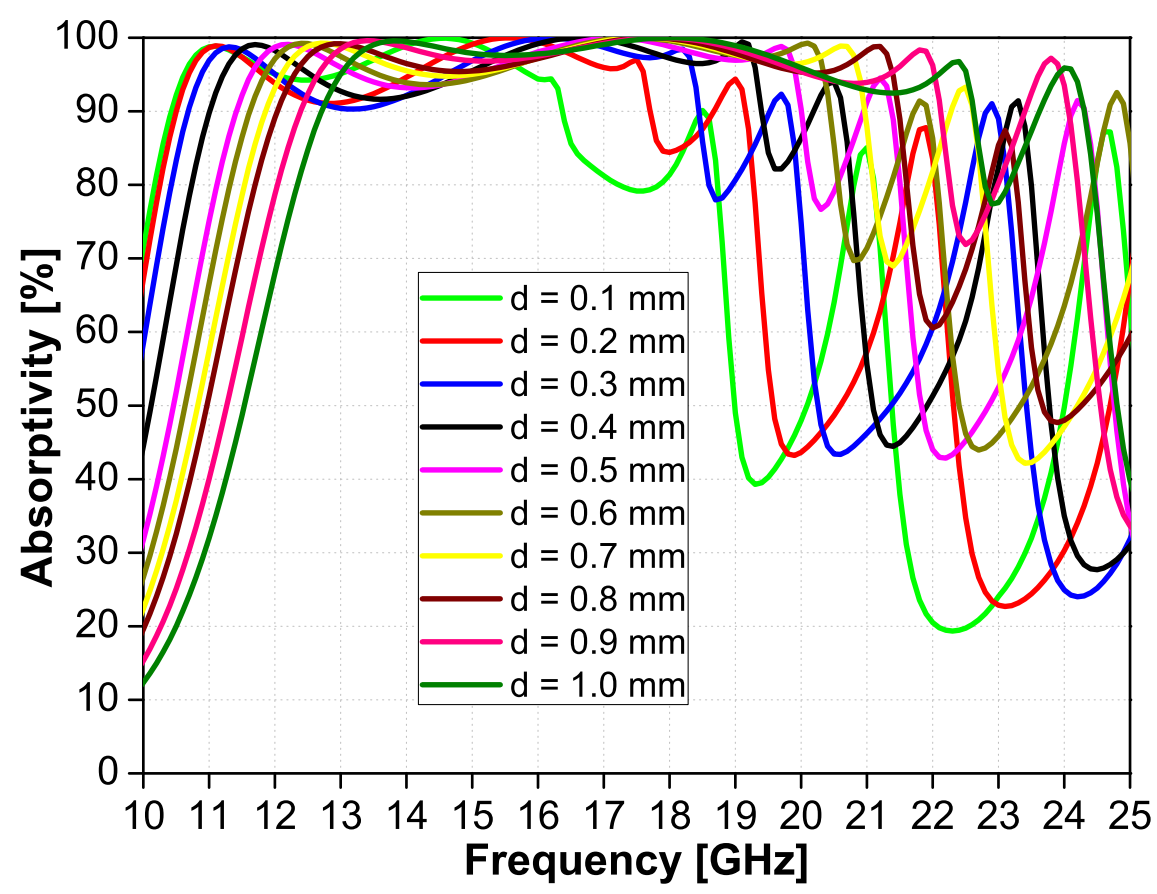

Figure 4.9: Absorptivity of the metamaterial absorber based on Model 4 considering different values of $d$.

In order to provide a better understanding of the absorption mechanism of the proposed metamaterial, the surface current distributions on the top and the bottom of the structure at $12.2 \mathrm{GHz}, 17.1 \mathrm{GHz}$ and $19.7 \mathrm{GHz}$ are plotted, as can be seen in Figure 4.10. This plot is important to verify the presence of electric and magnetic dipoles, which supply the electric and magnetic resonance responses. One of the main indicators of magnetic responses in metamaterials is the presence of antiparallel currents between the top and bottom layers, however, the proposed absorber does not present this feature at the analyzed frequencies. On the other hand, it is possible to note that the current densities in some adjacent strips are antiparallel, which indicates the presence of circulating current loops on the top of the absorber, and, thus, the presence of magnetic resonance is identified at 17.1 and $19.7 \mathrm{GHz}$. Furthermore, one can notice the presence of parallel currents between adjacent segments of the spiral at the three frequencies at issue. These parallel currents account to the electric resonance of the structure, once it is supplied by an electric-like dipole behavior [59]. At $12.2 \mathrm{GHz}$ is possible to observe parallelism between the currents on the inner parts of the spiral and the ground plane, which accounts to the electric resonance as well.

The electric and magnetic field distributions of the proposed absorber at 12.2 GHz, 17.1 GHz, and 19.7 GHz, as depicted in Figure 4.11, are important results that are able to provide valuable information about the mechanism of 
Table 4.2: Comparison of the proposed absorber with previously presented absorbers.

\begin{tabular}{|c|c|c|c|c|c|c|}
\hline \multirow{2}{*}{ Ref. } & \multirow{2}{*}{$\begin{array}{c}\text { Bandwidth } \\
(\mathrm{GHz})\end{array}$} & \multirow{2}{*}{$\begin{array}{c}\text { Relative } \\
\text { Bandwidth }\end{array}$} & \multicolumn{2}{|c|}{ Independent Incident Angle } & \multirow{2}{*}{ Thickness } & \multirow{2}{*}{ Periodicity } \\
\hline & & & TE & TM & & \\
\hline $\begin{array}{l}\text { Proposed } \\
\text { Structure }\end{array}$ & $11.44-20.00$ & $54.45 \%$ & $40^{\circ}$ (above $80 \%$ ) & $50^{\circ}$ (above $80 \%$ ) & $\begin{array}{c}1.6 \mathrm{~mm} \\
\left(0.061 \lambda_{\mathrm{L}}\right)\end{array}$ & $\begin{array}{l}3.655 \mathrm{~mm} \\
\left(0.139 \lambda_{\mathrm{L}}\right)\end{array}$ \\
\hline$[53]$ & $10.45-17.64$ & $51.19 \%$ & $40^{\circ}$ (above $70 \%$ ) & $40^{\circ}$ (above $70 \%$ ) & $\begin{array}{c}1.6 \mathrm{~mm} \\
\left(0.056 \lambda_{\mathrm{L}}\right)\end{array}$ & $\begin{array}{l}5.5 \mathrm{~mm} \\
\left(0.19 \lambda_{\mathrm{L}}\right)\end{array}$ \\
\hline$[54]$ & $7.85-12.25$ & $43.78 \%$ & $15^{\circ}$ (above $80 \%$ ) & $15^{\circ}$ (above $80 \%$ ) & $\begin{array}{c}2 \mathrm{~mm} \\
\left(0.052 \lambda_{\mathrm{L}}\right)\end{array}$ & $\begin{array}{l}7.1 \mathrm{~mm} \\
\left(0.19 \lambda_{\mathrm{L}}\right)\end{array}$ \\
\hline$[55]$ & $8.00-13.00$ & $47.62 \%$ & $30^{\circ}$ (above $90 \%$ ) & $30^{\circ}$ (above $90 \%$ ) & $\begin{array}{c}3 \mathrm{~mm} \\
\left(0.080 \lambda_{\mathrm{L}}\right)\end{array}$ & $\begin{array}{l}15.5 \mathrm{~mm} \\
\left(0.41 \lambda_{\mathrm{L}}\right)\end{array}$ \\
\hline$[56]$ & $7.87-12.43$ & $44.93 \%$ & $50^{\circ}$ (above $90 \%$ ) & $50^{\circ}$ (above $90 \%$ ) & $\begin{array}{c}5.6 \mathrm{~mm} \\
\left(0.147 \lambda_{\mathrm{L}}\right)\end{array}$ & $\begin{array}{l}13.8 \mathrm{~mm} \\
\left(0.362 \lambda_{\mathrm{L}}\right) \\
\end{array}$ \\
\hline$[57]$ & $7.50-12.80$ & $52.22 \%$ & $30^{\circ}$ (above $90 \%$ ) & $30^{\circ}$ (above $90 \%$ ) & $\begin{array}{c}3.0 \mathrm{~mm} \\
\left(0.075 \lambda_{\mathrm{L}}\right)\end{array}$ & $\begin{array}{l}11.0 \mathrm{~mm} \\
\left(0.275 \lambda_{\mathrm{L}}\right)\end{array}$ \\
\hline$[52]$ & $7.93-17.18$ & $73.98 \%$ & $40^{\circ}$ (above $80 \%$ ) & $40^{\circ}$ (above $80 \%$ ) & $\begin{array}{c}3.0 \mathrm{~mm} \\
\left(0.079 \lambda_{\mathrm{L}}\right)\end{array}$ & $\begin{array}{l}12.0 \mathrm{~mm} \\
\left(0.317 \lambda_{\mathrm{L}}\right)\end{array}$ \\
\hline$[58]$ & $1.35-3.50$ & $88.65 \%$ & $40^{\circ}$ (above $90 \%$ ) & $40^{\circ}$ (above $90 \%$ ) & $\begin{array}{l}21.6 \mathrm{~mm} \\
\left(0.097 \lambda_{\mathrm{L}}\right)\end{array}$ & $\begin{array}{c}20 \mathrm{~mm} \\
\left(0.090 \lambda_{\mathrm{L}}\right)\end{array}$ \\
\hline
\end{tabular}

absorption of the structure. In the electric field plots, the red region denotes the excited positive charges and the blue region, negative charges [60]. One can realize charges oscillations at the three analyzed frequencies, which provide evidence of electric resonance. Furthermore, at $19.7 \mathrm{GHz}$, there are strong accumulations of E-field coupling between adjacent segments in the outer turns of the scatter, which indicate the presence of displacement currents, and, thus, demonstrating the presence of the magnetic resonance [41]. Accordingly, the displacement current between the adjacent strips and the electric dipoles coupled with the top metallic layer indicate the presence of both electric and magnetic resonances. This conclusion matches with the surface current density plot, and, thus, these two types of resonances contribute to the characteristic absorption profile of the proposed structure, so the broadband of absorption is achieved by the overlap of these resonances over the frequency band of absorption. Hence, the absorption in the proposed metamaterial is driven by both magnetic and electric fields. It is important to note that in Figure 4.3, which presents the surface current distribution for a square spiral absorber without the gaps, the presence of circulating loop of current between the scatter and the ground plane are predominant, and, hence, showing that the initially considered absorber is driven by the magnetic field. Therefore, the presence of the gaps, as expected, increases the influence of the electric field in the absorption profile of the structure, broadening the bandwidth of absorption.

Although the plots of the surface current density and electric fields are efficient to indicate the presence the magnetic and/or electric resonance in the absorber, they are not enough to designate whether the proposed structure is driven by electric and/or magnetic field. To solve this issue, the effective 


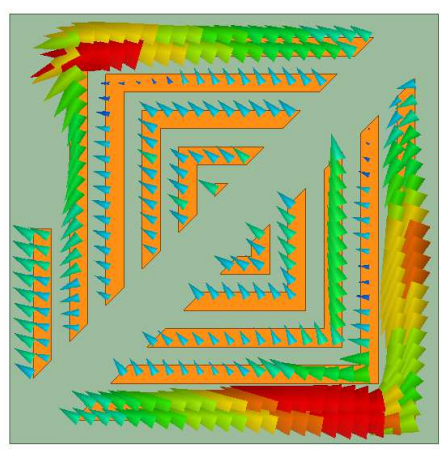

(a)

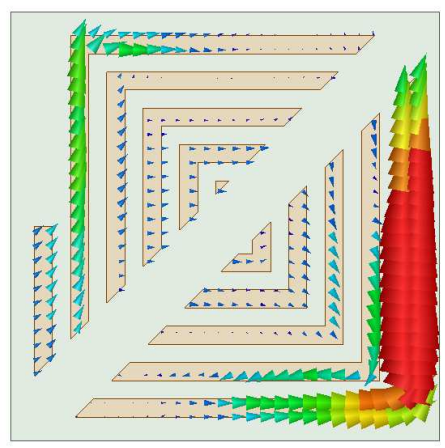

(b)

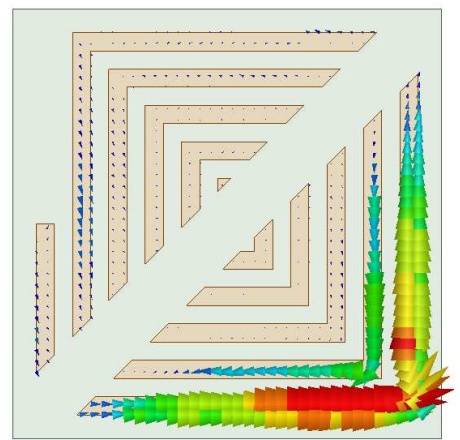

(c)

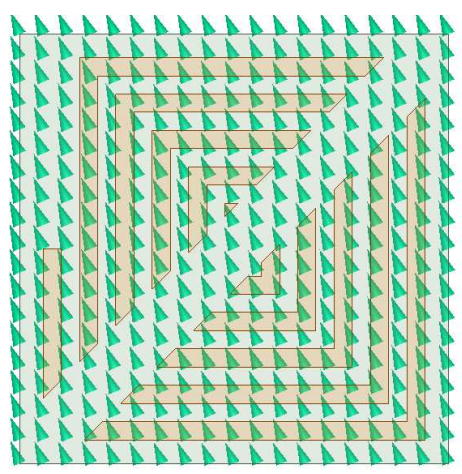

(d)

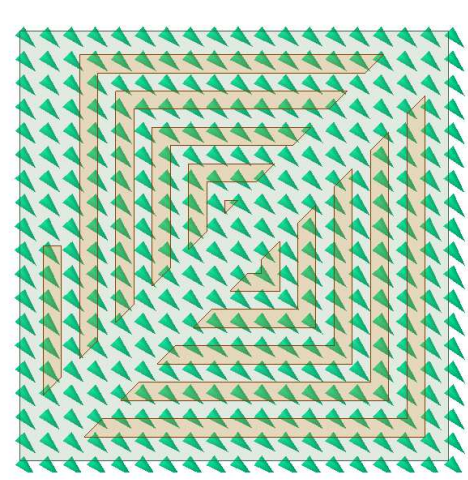

(e)

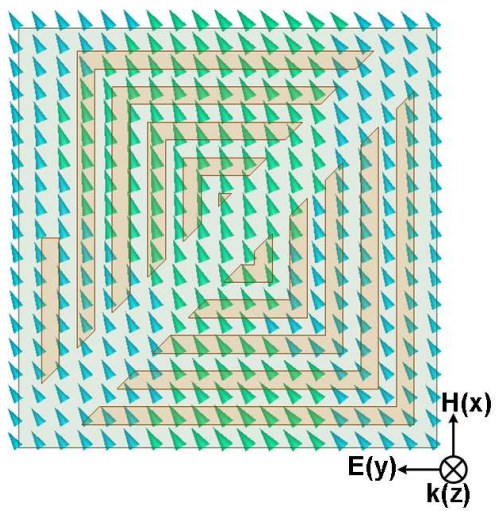

Jsurf

600.0

540.0

480.0

420.0

360.0

300.0

240.0

180.9

120.0

60.9

(f)

Figure 4.10: Simulated surface current density of the proposed metamaterial absorber on the top at (a) $12.2 \mathrm{GHz}$, (b) $17.1 \mathrm{GHz}$, and (c) $19.7 \mathrm{GHz}$, and on the bottom at (d) $12.2 \mathrm{GHz}$, (e) $17.1 \mathrm{GHz}$, and (f) $19.7 \mathrm{GHz}$.

permittivity $\epsilon_{\text {eff }}$ and permeability $\mu_{\text {eff }}$ are used to complement the information provided by the field plots. In order to extract the constitutive electromagnetic parameters of the proposed absorber, the method for characterizing a metasurface presented in Subsection 2.7.7 is employed. Figure 4.12 shows the real and imaginary parts of the effective permittivity, permeability, and the 


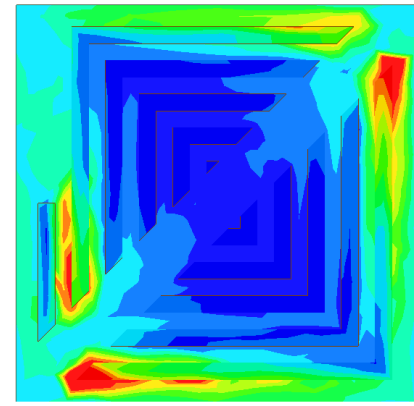

(a)

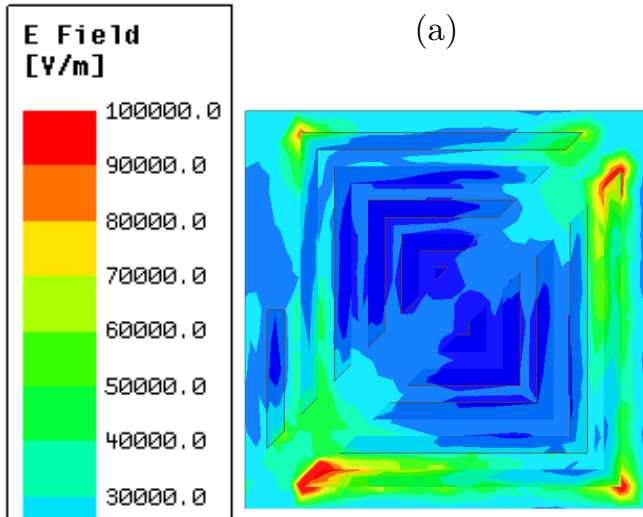

(b)

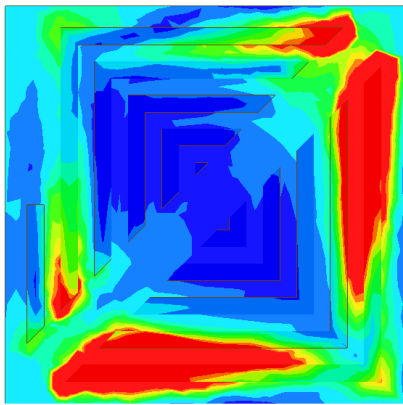

(c)

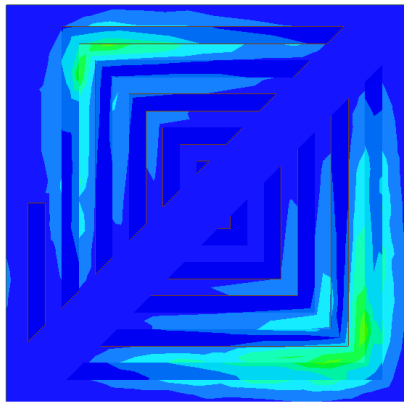

(d)

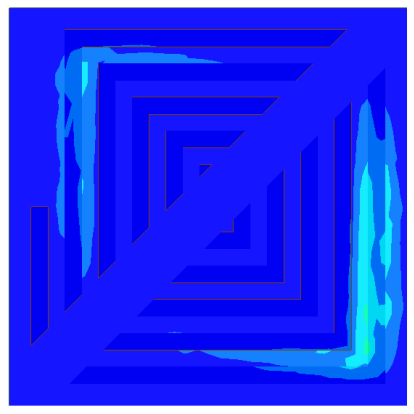

(e)

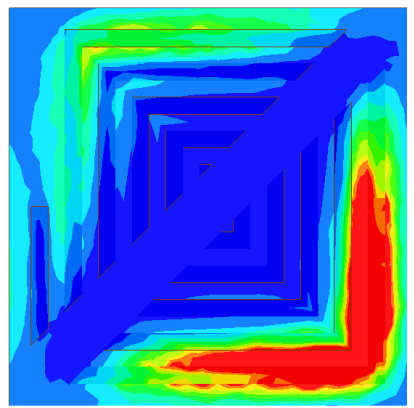

(f)

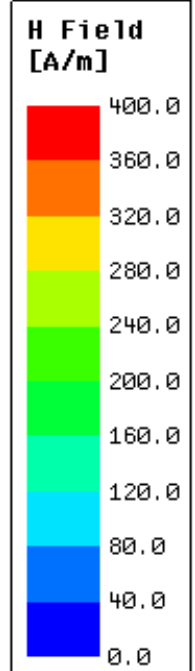

Figure 4.11: Electric field distribution of the proposed absorber at (a) 12.2 $\mathrm{GHz}$, (b) $17.1 \mathrm{GHz}$, and (c) $19.7 \mathrm{GHz}$, and the magnetic field distribution at (d) $12.2 \mathrm{GHz}$, (e) $17.1 \mathrm{GHz}$, and (f) $19.7 \mathrm{GHz}$.

refractive index. To begin with, it is possible to observe that over the bandwidth of absorption, which is from 11.44 to $20.00 \mathrm{GHz}$, the real part of the the effective permittivity $\epsilon_{\text {eff }}$ is mainly negative at the lower frequencies, which implies that there is a response to the external applied electric field, and, thus, the induced electric field opposes to the applied electric field at this frequency range [61]. On the other hand, the real part of $\mu_{\mathrm{eff}}$ is basically positive at the lower frequencies and negative at the highest frequencies, which means that both magnetic and electric resonance contributes to the absorption. Therefore, it is possible to conclude that the proposed absorber is driven by both electric and magnetic fields. The imaginary parts of both $\epsilon_{\mathrm{eff}}$ and $\mu_{\mathrm{eff}}$ are positives and 
present large variations over the bandwidth of absorption, which shows that losses are occurring in the structure, as expected. In addition, the real part of the refractive index varies slightly around 1, which shows an impedance matching between the free-space and the structure. Additionally, the imaginary part of the refractive index is related to the absorption experimented by the wave in the medium and is often called extinction coefficient. Its value is positive over the frequency range analyzed, which means that the absorption is happening.

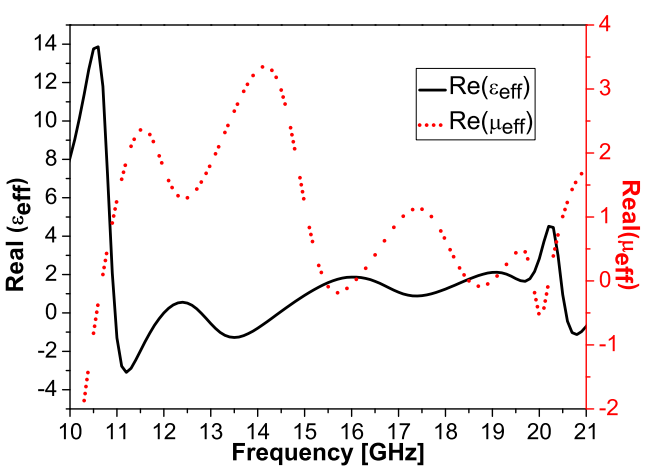

(a)

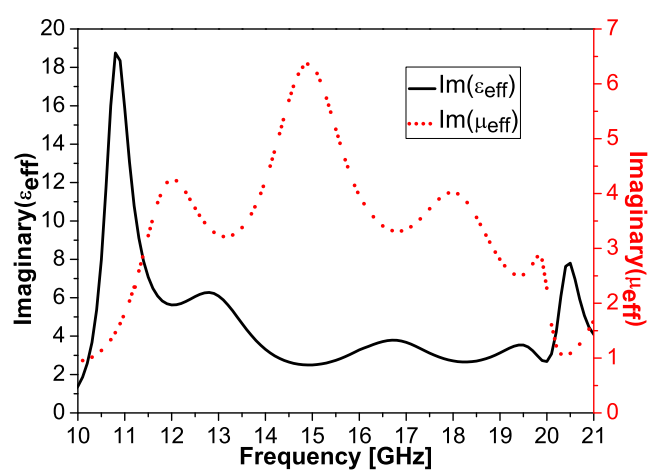

(b)

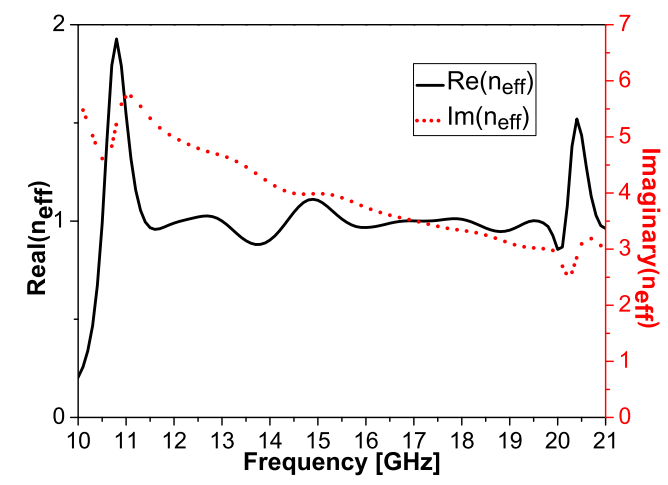

(c)

Figure 4.12: Extracted constitutive electromagnetic parameters: real (a) and imaginary (b) parts of $\epsilon_{\text {eff }}$ and $\mu_{\text {eff }}$, and the real and imaginary parts of $n_{\text {eff }}$ (c).

In Subsection 3.2.3, the mechanisms of absorption were discussed, and it is mentioned that there are two main types of losses in electromagneticwave absorbers, which are the ohmic and dielectric ones. To provide a better understanding of which type of absorption is dominant in the proposed absorber, one can plot the curve of absorptivity of two absorbers with the same dimensions, but considering a lossy and lossless substrate. So, Figure 4.13 shows the absorptivity of these two models. It is possible to note that the curves of absorption of the metamaterials are almost identical over the main frequency band of absorption, which means that the dielectric losses are insignificant when compared to the ohmic ones. Moreover, this feature implies 
that the performance of the structure does not depend exclusively on the type of dielectric substrate, which provides huge design flexibility.

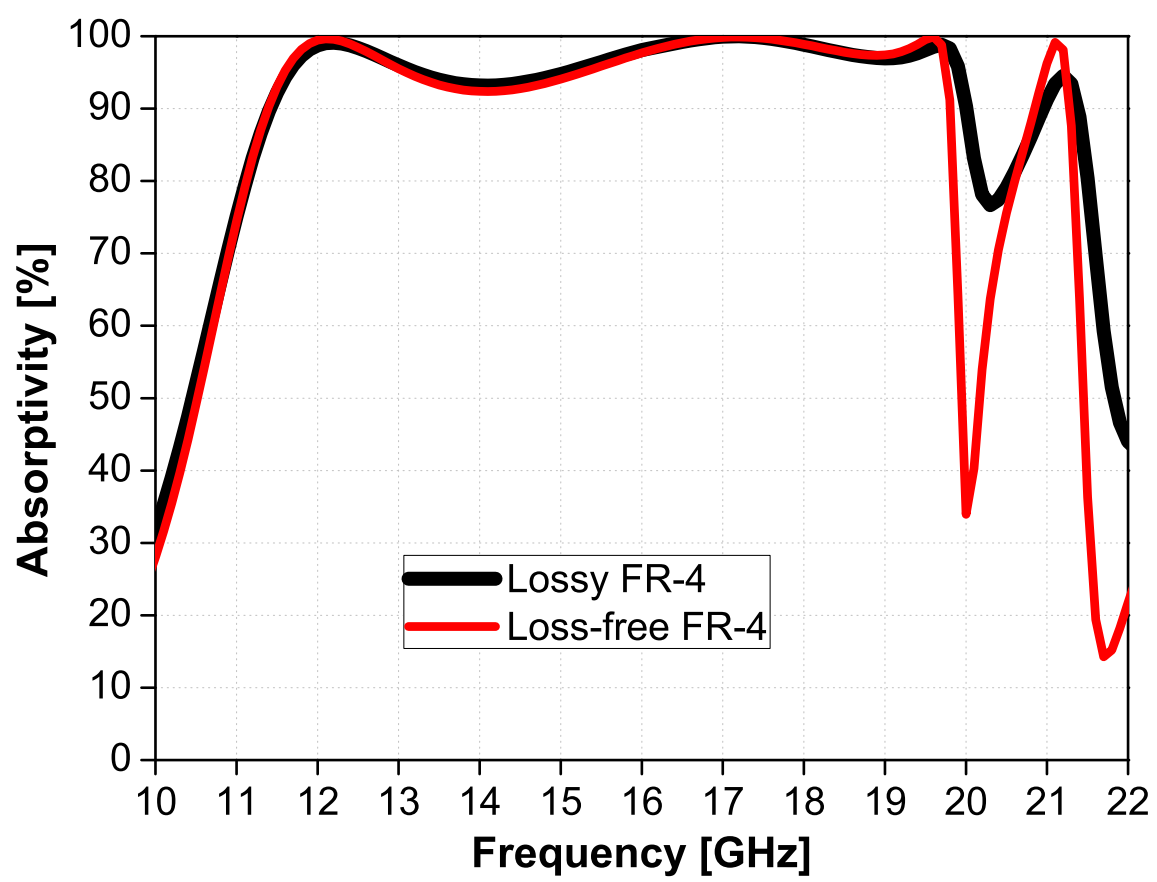

Figure 4.13: Absorptivity of the proposed absorber considering lossy and lossfree FR-4 substrates.

Since the losses in the proposed absorber are mainly ohmic, a great flexibility in the frequency band of absorption can be achieved. In order to prove this statement, the absorptivity of the metamaterial considering the same thickness and dimensions, as the model 1 in Table 4.1, but with different electromagnetic properties, as can be seen in Figure 4.14. It is considered four different substrates: FR-4 $\left(\epsilon_{r}=4.4\right)$, Rogers $5880\left(\epsilon_{r}=2.2\right)$, air $\left(\epsilon_{r}=1\right)$, and RO $3006\left(\epsilon_{r}=6.15\right)$. Moreover, it is possible to observe that the relative permittivity is inversely proportional to the central frequency of absorption and that the broadband feature is maintained regardless of the $\epsilon_{r}$. To summarize the results of the absorbers with different substrates, Table 4.3 shows their bandwidth, relative bandwidth, and central frequency of absorption, which are calculated considering the absorptivity above 90\%. One can notice that the relative broadband is high for the different substrates, which shows the great flexibility of the design based on square spiral with gaps. Furthermore, this feature combined with the sensibility to the variations of the thickness, as can be observed in Figure 4.5, demonstrates the great capacity of adaptation of the proposed absorber to different applications for a variety of frequency bands. 


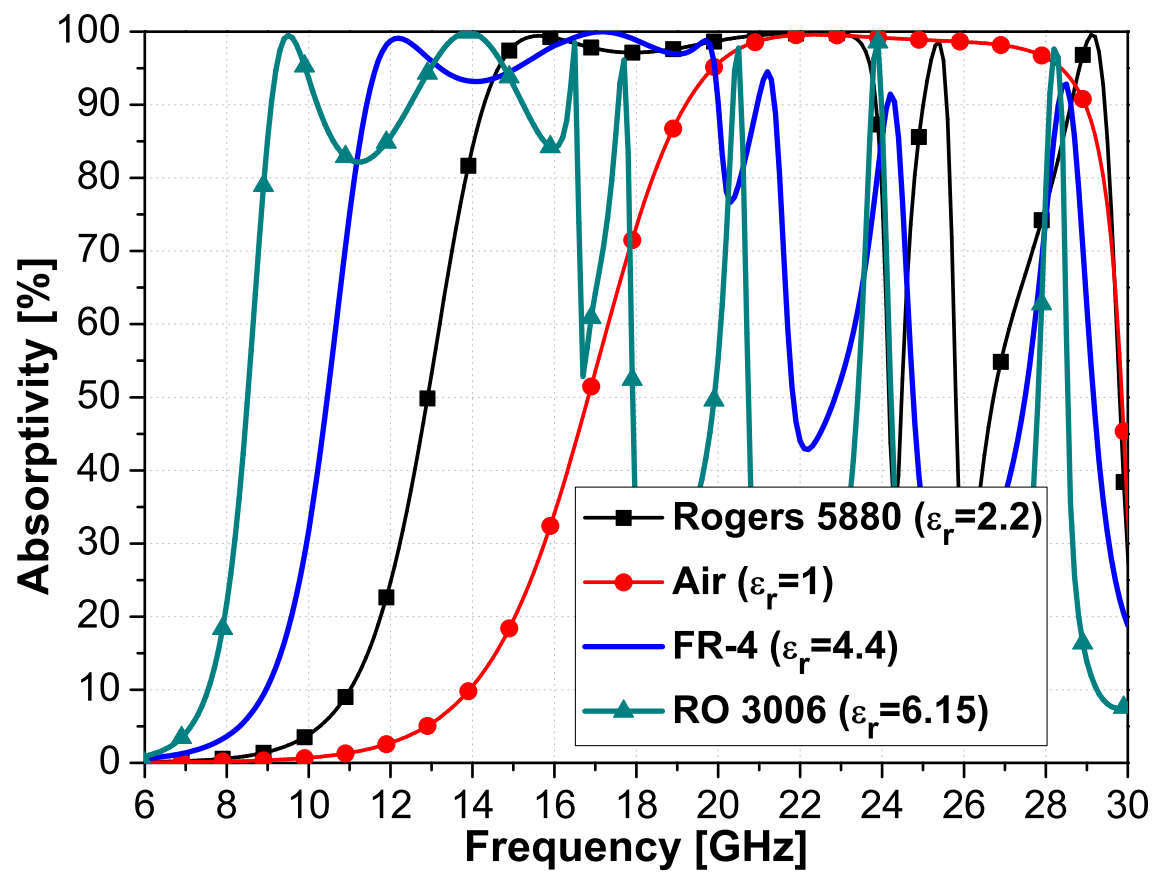

Figure 4.14: Absorptivity of the proposed absorber considering different substrates.

Table 4.3: Results of the proposed absorber considering different substrates.

\begin{tabular}{|c|c|c|c|c|}
\hline Substrate & $\epsilon_{r}$ & $\begin{array}{c}\text { Bandwidth } \\
(\mathbf{G H z})\end{array}$ & $\begin{array}{c}\text { Relative } \\
\text { Bandwidth }\end{array}$ & $\begin{array}{c}\text { Central } \\
\text { Frequency (GHz) }\end{array}$ \\
\hline RO 3006 & 6.15 & $9.02-15.24$ & $51.28 \%$ & 12.13 \\
\hline FR-4 & 4.4 & $11.44-20.00$ & $54.45 \%$ & 15.72 \\
\hline Rogers 5880 & 2.2 & $14.28-23.85$ & $51.20 \%$ & 19.07 \\
\hline Air & 1 & $19.20-28.96$ & $40.20 \%$ & 24.03 \\
\hline
\end{tabular}

4.3

Equivalent Circuit and Parameter Retrieval

In this Section, an equivalent circuit and a original parameter retrieval method are introduced to describe broadband electromagnetic-wave absorbers. The equivalent circuit of absorbers is an important tool for their analyses and optimizations, once it provides information regarding the resonance characteristic of the structure. However, some works used to defined the equivalent circuit and their parameters without showing how their values were found, limiting their equivalent circuit and analysis to a particular structure [55], [62]. Besides that, it is possible to find some parameter retrieval methods for classic structures such as the Jerusalem cross [46] and the patch [47], or for narrowband absorbers [63]. Nevertheless, when it comes to broadband absorbers with arbitrary shapes, there is a lack of equivalent circuits and parameter extraction methods described in details in the literature. 
To describe absorbers as an equivalent circuit, LC-like circuits have been used extensively in the literature [63], [64], [45], due to their accuracy and straightforward analysis. Furthermore, a broadband metamaterial absorber is usually a multi-resonant structure with a high level of absorption between the peaks of resonance, which means that this kind of absorber requires multiples series LC with parallel connections [44]. Taking this into account, the equivalent circuit for broadband absorbers is initially proposed, as can be seen in Figure 4.15. One can notice that there are other elements in the circuit besides the series LC, which are the resistor $R_{d}$ and the inductor $L_{d}$. The presence of $R_{d}$ is necessary to represent the losses in the employed substrate, whereas $L_{d}$ represents the inductive behavior of the grounded thin substrate.

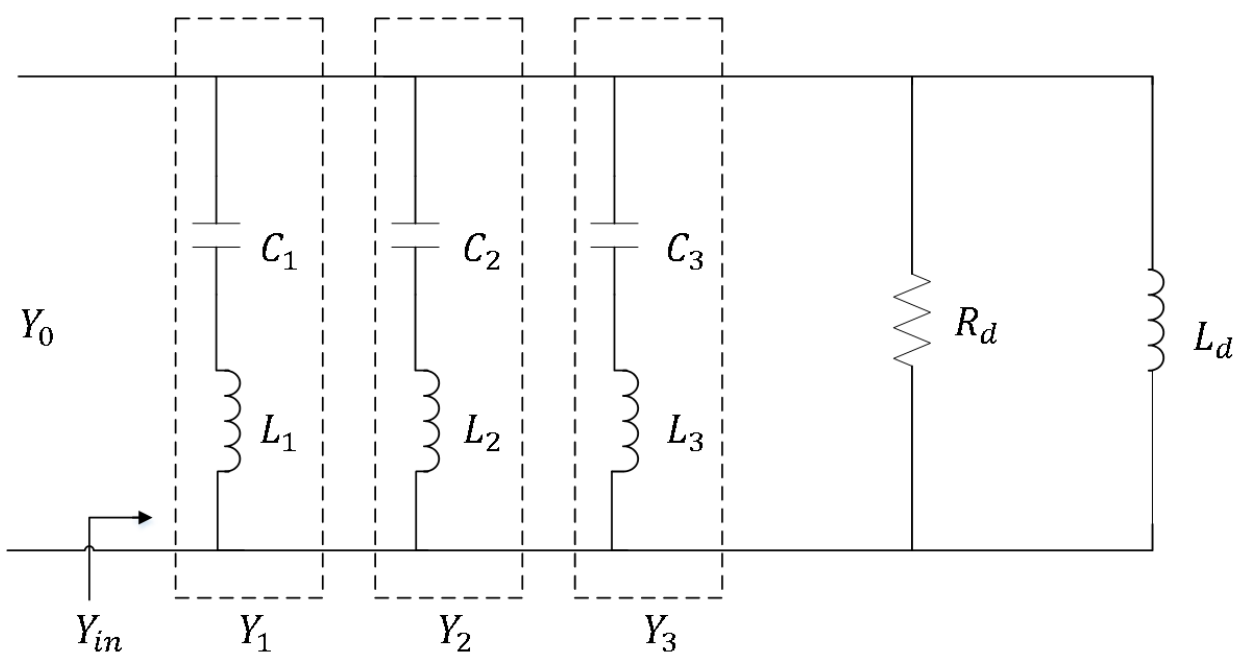

Figure 4.15: Equivalent circuit of a broadband metamaterial absorber.

The input admittance of the equivalent circuit in Figure 4.15 is written as follows

$$
Y_{\text {in }}=Y_{1}+Y_{2}+Y_{3}+Y_{d}+1 / R_{d}=a_{y}+j b_{y},
$$

where $a_{y}$ and $b_{y}$ are the real and imaginary parts of the input admittance of the absorber, respectively, and

$$
\begin{gathered}
Y_{n}=j\left(\frac{\omega C_{n}}{1-\omega^{2} L_{n} C_{n}}\right), n=[1,2,3], \\
Y_{d}=\frac{1}{j \omega L_{d}} .
\end{gathered}
$$

It is important to point out that the input admittance of the absorber can be extracted directly from the $S_{11}$ from the EM simulation using the following relation

$$
Y_{\mathrm{in}}=Y_{0} \frac{1-S_{11}}{1+S_{11}},
$$


where $Y_{0}$ is free-space admittance. In addition, to provide a reference of the input admittance and/or impedance obtained from the EM simulation of the proposed absorber (Model 1), Figure 4.16 shows their real and imaginary parts. It is possible to note that the real part of the impedance varies over the frequency band of absorption, but its highest value is $600 \Omega$. Also, the imaginary part demonstrates a capacitive behavior, i.e. capacitive reactance, from 11.0 to $15.1 \mathrm{GHz}$, and from 15.1 to $21.5 \mathrm{GHz}$ is possible to note that the absorber presents an inductive reactance, as expected.

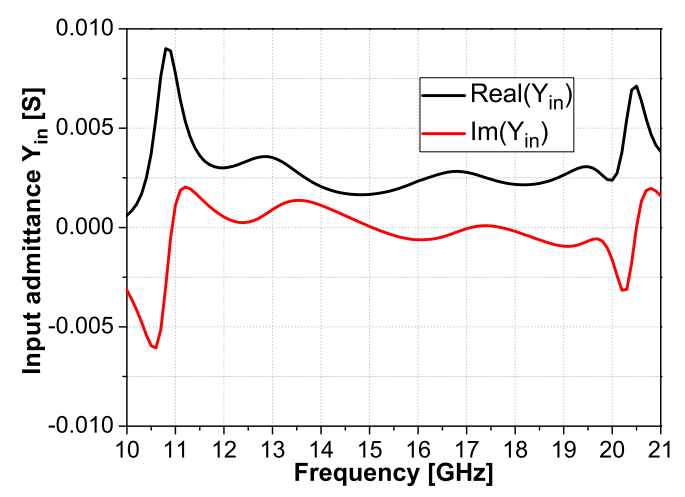

(a)

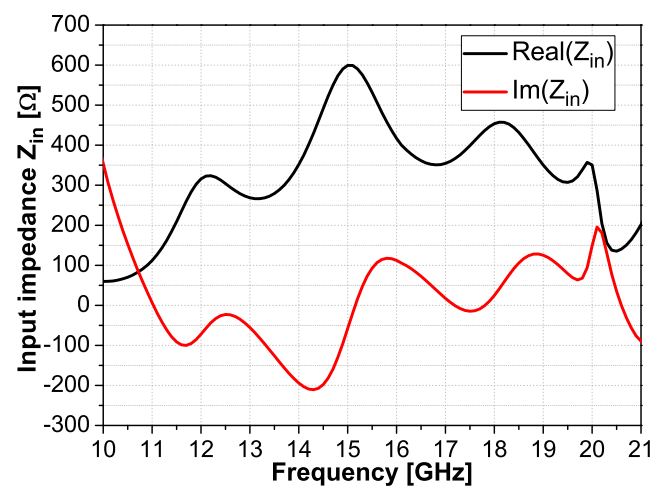

(b)

Figure 4.16: Real and imaginary parts of the (a) admittance and (b) impedance of the proposed absorber from the EM simulation.

To describe the equivalent circuit, the input admittance calculated from Equation 4-1 must be as similar as possible from the one numerically calculated on the full-wave analysis. Furthermore, to fulfill the condition presented in Equation 3-14, the initial value of $R_{d}$ is $377 \Omega$, and, therefore, trying to provide an impedance matching at the interface between the structure and the freespace. Nevertheless, the extraction of $C_{n}, L_{n}$, and $L_{d}$ is not straightforward and requires a deeper mathematical development of the imaginary part of the input admittance, which can be written as follows

$$
\begin{aligned}
b_{y}=\omega C_{1}\left(1-\omega^{2} L_{2} C_{2}\right)\left(1-\omega^{2} L_{3} C_{3}\right) \omega L_{d} \\
+\omega C_{2}\left(1-\omega^{2} L_{1} C_{1}\right)\left(1-\omega^{2} L_{3} C_{3}\right) \omega L_{d} \\
\quad+\omega C_{3}\left(1-\omega^{2} L_{1} C_{1}\right)\left(1-\omega^{2} L_{2} C_{2}\right) \omega L_{d} \\
\left.-\left(1-\omega^{2} L_{1} C_{1}\right)\left(1-\omega^{2} L_{2} C_{2}\right)\left(1-\omega^{2} L_{3} C_{3}\right)\right] \\
\times \\
\quad\left[\left(1-\omega^{2} L_{1} C_{1}\right)\left(1-\omega^{2} L_{2} C_{2}\right)\left(1-\omega^{2} L_{3} C_{3}\right) \omega L_{d}\right]^{-1} .
\end{aligned}
$$

In order to find a linear equation for the imaginary part of the input admittance $b_{y}$, the Equation 4-5 can be mathematically rearranged into 


$$
1=-\omega b_{y} \alpha+\omega^{2} \beta+\omega^{3} b_{y} \gamma-\omega^{4} \nu-\omega^{5} b_{y} \delta+\omega^{6} \kappa+\omega^{7} b_{y} \rho
$$

where

$$
\begin{gathered}
\alpha=L_{d}, \\
\beta=C_{1} L_{d}+C_{2} L_{d}+C_{3} L_{d}+C_{1} L_{1}+C_{2} L_{2}+C_{3} L_{3}, \\
\gamma=C_{1} L_{1} L_{d}+C_{2} L_{2} L_{d}+C_{3} L_{3} L_{d}, \\
\nu=L_{2} C_{2} C_{1} L_{d}+L_{3} C_{3} C_{1} L_{d}+L_{3} C_{3} C_{2} L_{d}+ \\
+L_{1} C_{1} C_{2} L_{d}+L_{2} C_{2} C_{3} L_{d}+L_{1} C_{1} C_{3} L_{d}+ \\
+L_{2} C_{2} L_{3} C_{3}+L_{1} C_{1} L_{3} C_{3}+L_{1} C_{1} L_{2} C_{2}, \\
\delta=C_{1} L_{1} C_{2} L_{2} L_{d}+C_{1} L_{1} C_{3} L_{3} L_{d}+C_{2} L_{2} C_{3} L_{3} L_{d}, \\
\kappa=L_{2} C_{2} L_{3} C_{3} C_{1} L_{d}+L_{1} C_{1} L_{3} C_{3} C_{2} L_{d}+ \\
+L_{1} C_{1} L_{2} C_{2} C_{3} L_{d}+L_{1} C_{1} L_{2} C_{2} L_{3} C_{3}, \\
\rho=L_{1} C_{1} L_{2} C_{2} L_{3} C_{3} L_{d} .
\end{gathered}
$$

The Equation 4-6 can be written in a matricial form, which is represented as

$$
\left[\begin{array}{c}
1 \\
1 \\
\vdots \\
1
\end{array}\right]=\Phi\left[\begin{array}{c}
\alpha \\
\beta \\
\gamma \\
\nu \\
\delta \\
\kappa \\
\rho
\end{array}\right],
$$

where $\Phi$ is an $n \times 7$ matrix and is represented by

$$
\Phi=\left[\begin{array}{ccccccc}
-\omega_{1} b_{y}\left(\omega_{1}\right) & \omega_{1}^{2} & \omega_{1}^{3} b_{y}\left(\omega_{1}\right) & -\omega_{1}^{4} & -\omega_{1}^{5} b_{y}\left(\omega_{1}\right) & \omega_{1}^{6} & \omega_{1}^{7} b_{y}\left(\omega_{1}\right) \\
-\omega_{2} b_{y}\left(\omega_{2}\right) & \omega_{2}^{2} & \omega_{2}^{3} b_{y}\left(\omega_{2}\right) & -\omega_{2}^{4} & -\omega_{2}^{5} b_{y}\left(\omega_{2}\right) & \omega_{2}^{6} & \omega_{2}^{7} b_{y}\left(\omega_{2}\right) \\
\vdots & \vdots & \vdots & \vdots & \vdots & \vdots & \vdots \\
-\omega_{m} b_{y}\left(\omega_{m}\right) & \omega_{m}^{2} & \omega_{m}^{3} b_{y}\left(\omega_{m}\right) & -\omega_{m}^{4} & -\omega_{m}^{5} b_{y}\left(\omega_{m}\right) & \omega_{m}^{6} & \omega_{m}^{7} b_{y}\left(\omega_{m}\right)
\end{array}\right]
$$

where $m$ is the number of measured data.

The Equation 4-14 is a system of linear equations of the form $\boldsymbol{A x}=\boldsymbol{b}$, where $\boldsymbol{A} \in \mathbb{R}^{m \times n}, \boldsymbol{b} \in \mathbb{R}^{m}, m \geq n$, and rank $\boldsymbol{A}=n$. In order to solve this 
kind of system, the least-square method is employed [65], in which its goal is to find the vector $x^{*}$ that minimizes $\|\boldsymbol{A} \boldsymbol{x}-\boldsymbol{b}\|^{2}$, for all $\boldsymbol{x} \in \mathbb{R}^{m}$. Furthermore, the vector $x^{*}$ is known as the least-square solution to $\boldsymbol{A} \boldsymbol{x}=\boldsymbol{b}$. So, using the least-square method, the Equation 4-14 can be solved as

$$
\left[\begin{array}{l}
\alpha \\
\beta \\
\gamma \\
\nu \\
\delta \\
\kappa \\
\rho
\end{array}\right]=\left(\Phi^{T} \Phi\right)^{-1} \Phi^{T}\left[\begin{array}{c}
1 \\
1 \\
\vdots \\
1
\end{array}\right],
$$

where the superscript " $T$ " indicates the transpose of the matrix.

Considering the absorber analysed in Section 4.1.1 with the admittance shown in Figure 4.16(a) and using the Equation 4-16, it is possible to find the values that better fit the curve of the equivalent circuit to the observed data, i.e. from the EM simulation. Hence, it is possible to find the following values $\alpha=4.6412 \cdot 10^{-9} \mathrm{H}, \beta=3.4064 \cdot 10^{-22} \mathrm{~F} \cdot \mathrm{H}, \gamma=1.2632 \cdot 10^{-30} \mathrm{~F} \cdot \mathrm{H}^{2}$, $\nu=3.6075 \cdot 10^{-44} \mathrm{~F}^{2} \cdot \mathrm{H}^{2}, \delta=9.5141 \cdot 10^{-53} \mathrm{~F}^{2} \cdot \mathrm{H}^{3}, \kappa=1.2177 \cdot 10^{-66} \mathrm{~F}^{3} \cdot \mathrm{H}^{3}$, and $\rho=1.8907 \cdot 10^{-75} \mathrm{~F}^{3} \cdot \mathrm{H}^{4}$. Furthermore, the optimal values of capacitance and inductance of the equivalent circuit are found solving the Equations 4-7 - 4-13 and their values are $L_{1}=10.71 \mu \mathrm{H}, C_{1}=1.49 \mathrm{fF}, L_{2}=1.98 \mu \mathrm{H}, C_{2}=408.17$ $\mathrm{fF}, L_{3}=2.39 \mathrm{nH}, C_{3}=13.22 \mathrm{fF}$, and $L_{d}=4.64 \mathrm{nH}$.

Using the optimal values of capacitance and inductance found and the value of $R_{d}$ initially estimated, which is $377 \Omega$, the curve of absorptivity of the equivalent circuit is shown in Figure 4.17. One can notice that there is a good matching between the bandwidth of absorption of both curves. However, it is possible to note two flaws in the absorption around 12.6 and $17.8 \mathrm{GHz}$.

To overcome problems related to the level of absorption, resistors must be added to the proper series LC to fix the two flaws in the absorption, once they can dissipate the impinging energy due to the ohmic losses. According to the values of resonance and inductance found, it is possible to note that the extra resistors must be added in $Y_{1}$ and $Y_{2}$, once their resonances occurs at $f_{1}=12.59 \mathrm{GHz}$ and $f_{2}=17.72 \mathrm{GHz}$, respectively. Therefore, the final equivalent circuit is proposed, as can be observed in Figure 4.18.

A curve fitting method is employed to find the optimal values of $R_{1}$ and $R_{2}$. The parameters found are $R_{1}=1010 \Omega$ and $R_{2}=10000 \Omega$, and the curve of absorptivity is shown in Figure 4.19. One can notice that the added resistors 


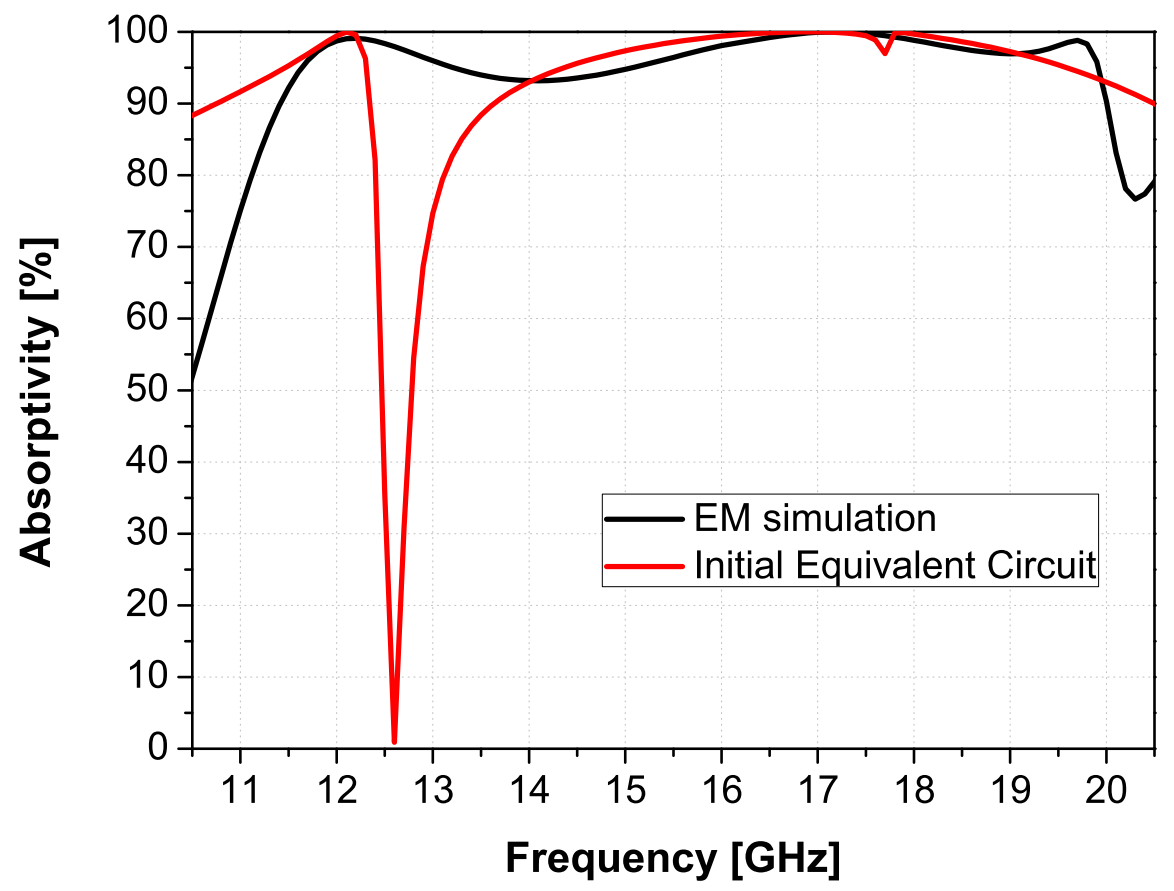

Figure 4.17: Curves of absorptivity of the absorber for the equivalent circuit and the EM simulation.

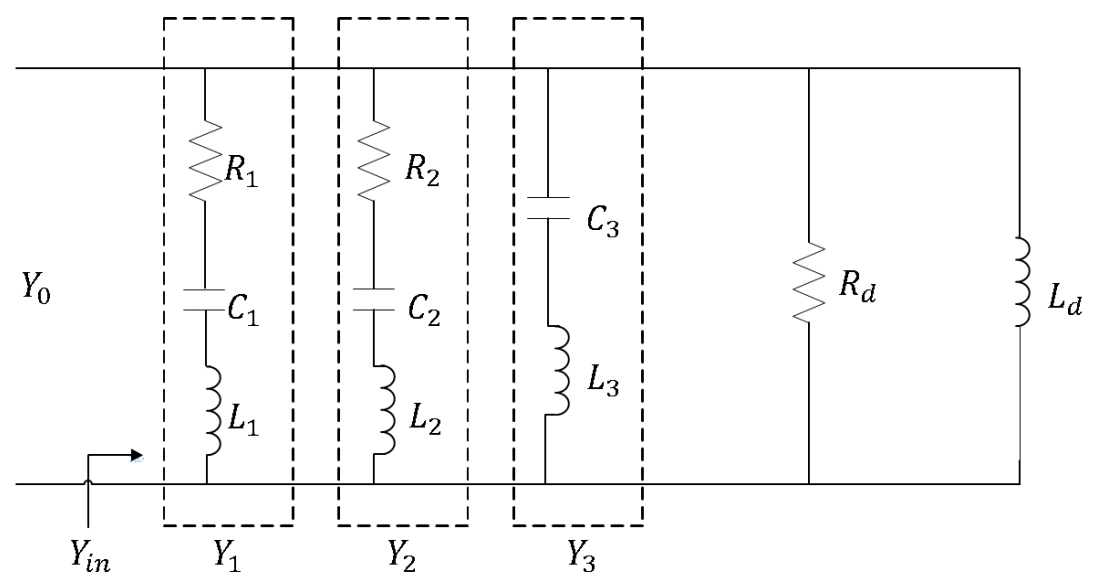

Figure 4.18: Final equivalent circuit for the proposed metamaterial absorber.

provide an increase in the absorption around 12.5 and $17.7 \mathrm{GHz}$, as expected. Moreover, in terms of level and bandwidth of absorption, the final equivalent circuit presents a good agreement with the absorption obtained in the EM simulation. The slight difference between these curves is expected, once their analyses consider different physical phenomena.

In order to provide an understanding of the effect of the insertion of extra resistors in the equivalent circuit, Figure 4.20 shows the input impedance of both initial and final equivalent circuits. One can notice that even with relatively high values of $R_{1}$ and $R_{2}$, the increase in the real part of the 


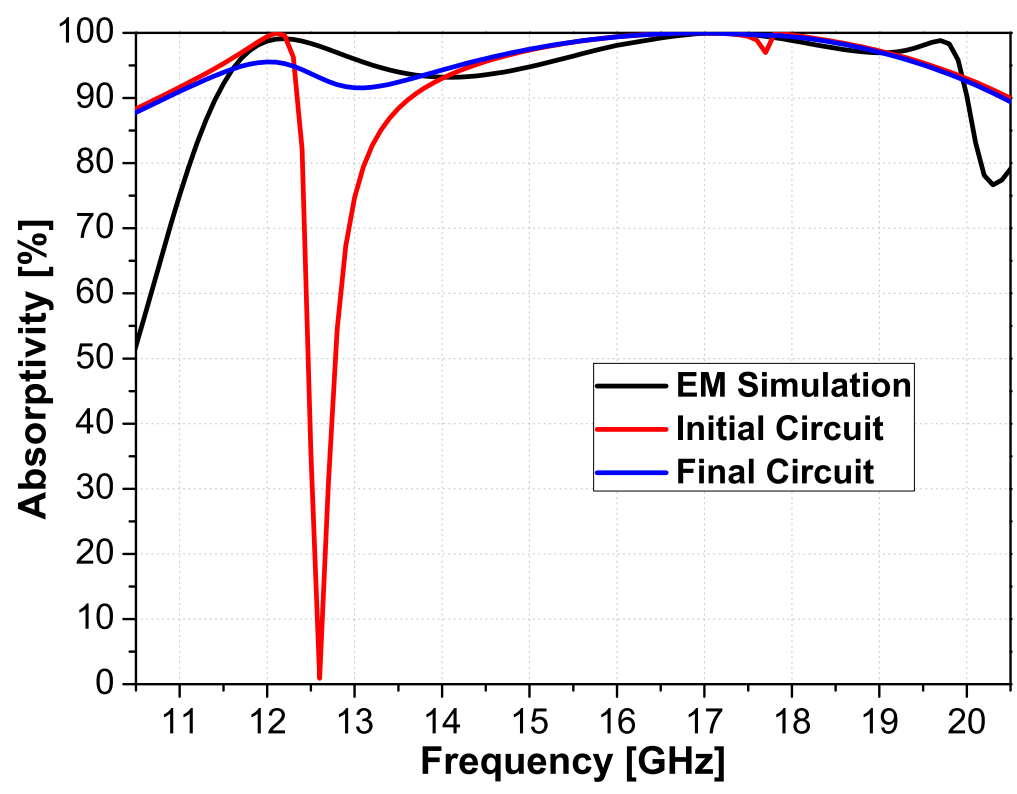

Figure 4.19: Curves of absorptivity for the initial and final equivalent circuit, and for the EM simulation.

impedance is not so high. Around $12.6 \mathrm{GHz}$, the real part of the impedance of the initial circuit is zero, and the efficiency of the presence of the $R_{1}$ is showed by the increase of the absorption at this frequency. On the other hand, the level of the imaginary part of the input impedance decreases with the addition of $R_{1}$ and $R_{2}$. This effect shows the importance of using an efficient algorithm of curve fitting to find the values of resistance for the final equivalent circuit. Furthermore, the curve of absorptivity from the EM simulation must be used as a reference for the curve fitting, and, thus, the real and imaginary parts of the input impedance balance themselves to find the optimal curve of absorption.

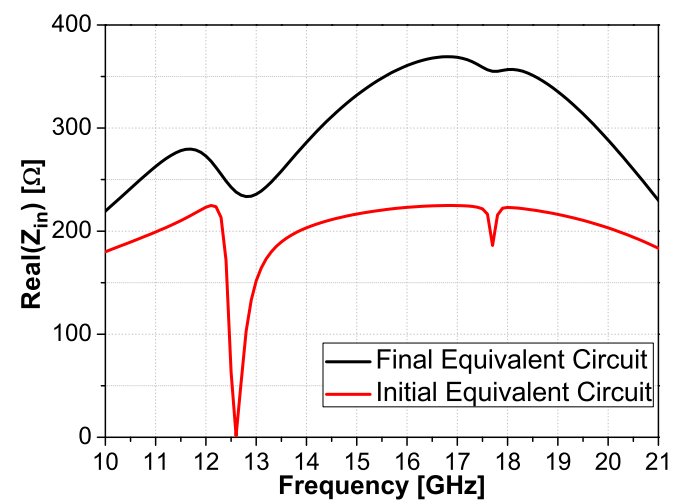

(a)

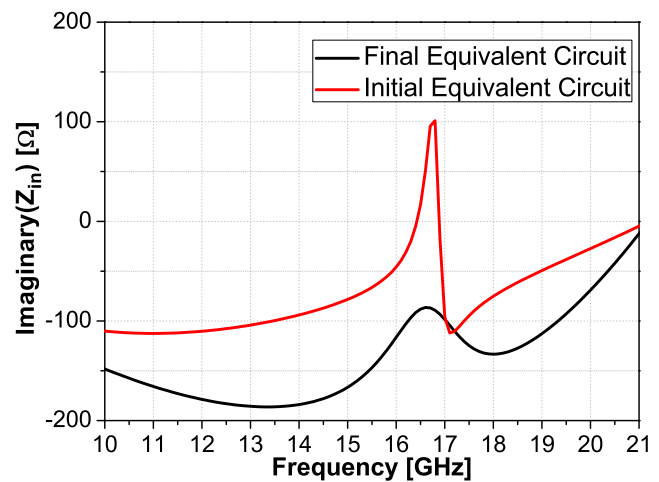

(b)

Figure 4.20: Real (a) and imaginary (b) parts of the input impedance from the initial and final equivalent circuits. 


\section{4}

\section{Applications of the Proposed Metamaterial Absorber}

In this Section, the usage of the proposed metamaterial absorber in antennas and arrays is investigated. The absorber is employed to reduce the RCS and mutual coupling between antenna-elements of an array. Also, the performance of the antenna and the influence of the presence of the metamaterial are discussed.

\subsection{1}

\section{Radar Cross-Section Reduction in Antennas}

The exponential development of stealth technology led researchers to study ways to reduce the RCS of antennas without compromising their performance. For this purpose, a variety of methods have been employed using a biased ferrite substrate [66], distributed loading [67], resistive sheets [68], and electromagnetic band-gap [69], to name a few. However, these techniques could increase the cost, volume, weight, and complexity of the antenna, which are characteristics avoided by modern applications. To overcome these drawbacks, metamaterial absorbers have been used due to their outstanding absorbing properties [70], [71], [72].

\subsubsection{1}

\section{RCS Analysis of a Microstrip Patch Antenna}

In this Subsection, the proposed antenna is designed to operate at the $\mathrm{X}$ band ( $8-12 \mathrm{GHz})$ as well as the employed metamaterial absorber. In addition, once the antenna and the absorber share the same host substrate, the material must be carefully chosen. It is well known that the FR-4 is not a good substrate for antennas operating at high frequencies such as the $\mathrm{X}$ band, due to its high losses. Taking this into account this property, the substrate Rogers RT/duroid 6006 , with $\epsilon_{r}$ equals to 6.15 and loss tangent of 0.0027 , is considered, which is ideal for the aimed frequency band.

At first, the metamaterial absorber is proposed to present absorption above $90 \%$ over the $\mathrm{X}$ band. The design is the same one employed throughout this Chapter, as can be seen in Figure 4.4. Furthermore, the substrate Rogers 6006 is considered with $1.9 \mathrm{~mm}$ of thickness and the dimensions of the absorber, in $\mathrm{mm}$, are $a=3.655, w=0.155, p=0.155, g=0.2, d=0.3$, and $h=1.9$. The curves of absorptivity of this metamaterial for both TE and TM polarization under normal incidence can be observed in Figure 4.21. One can note that the bandwidth of absorption above $90 \%$ goes from 7.96 to $12.24 \mathrm{GHz}$, covering the $\mathrm{X}$ band. Moreover, the curves are identical for both considered orthogonal 
polarization. It is important to point out that the relative bandwidth of the proposed absorber is $42.38 \%$ and its unit cell is extremely compact, once its periodicity and thickness are, in terms of the longest wavelength of the bandwidth of the absorption $\lambda_{L}, 0.097 \lambda_{L}$ and $0.05 \lambda_{L}$, respectively.

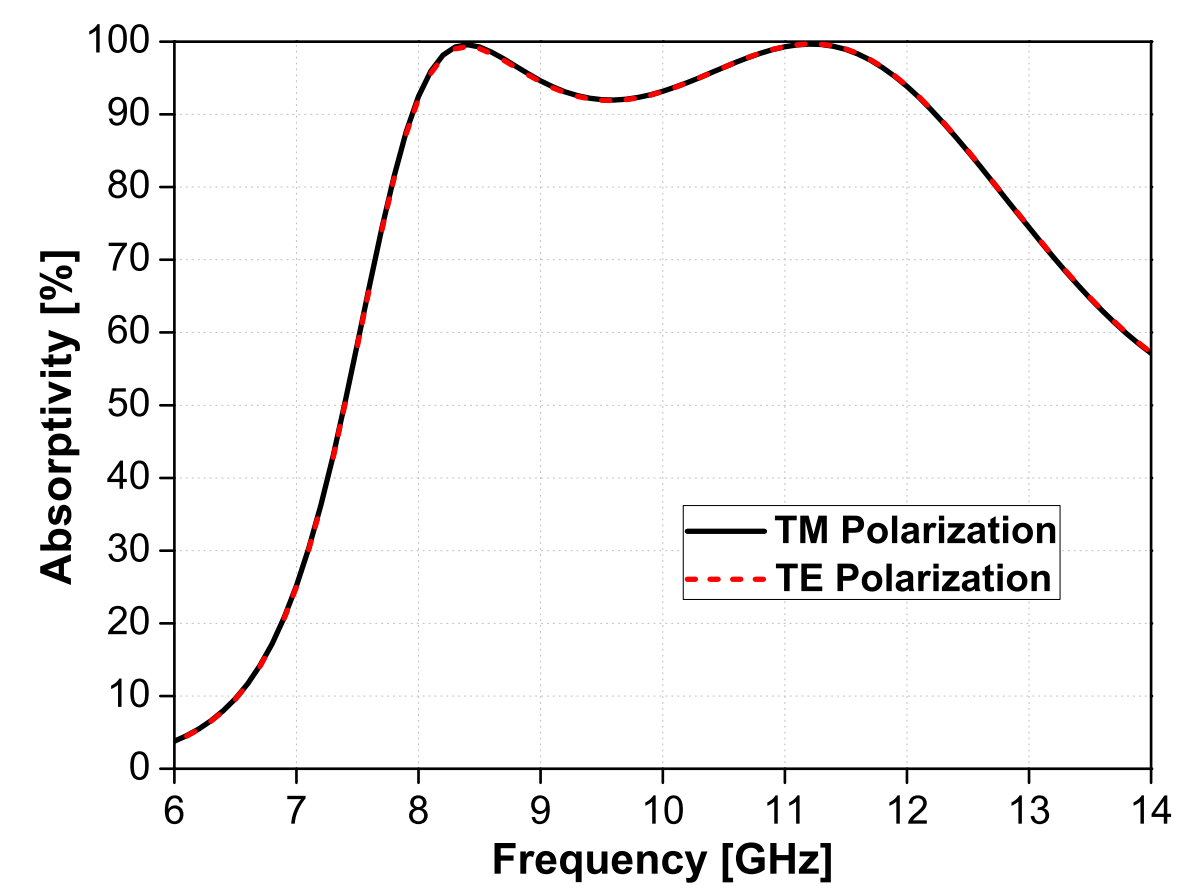

Figure 4.21: Curves of absorptivity for the absorber designed to operate at the $\mathrm{X}$ band.

A probe-fed microstrip antenna is considered to verify the efficiency of the absorber in the RCS reduction. The antenna is designed to operate at $10 \mathrm{GHz}$ and two rows of absorbers are placed around the patch, as can be seen in Figure 4.22, and its dimensions, in $\mathrm{mm}$, are $h=1.9, W_{g}=25.585$, $L_{g}=25.585, W=7.93, L=5.07, L_{\text {feed }}=4.85$, and $W_{\text {feed }}=W / 2$, in which the parameters $W_{\text {feed }}$ and $L_{\text {feed }}$ indicate the feeding position. Furthermore, the substrate Rogers 6006 is used and the minimum distance between the patch and a metamaterial unit cell is $1.5175 \mathrm{~mm}$, and the probe feed is designed to present $50 \Omega$ of impedance.

Figure 4.23 shows the reflection coefficient of the antenna with and without the metamaterial absorber. It is possible to note that the operating frequencies of both antennas are the same regardless of the presence of the absorber. Moreover, the antennas with and without the absorber present reflection coefficient at $10 \mathrm{GHz}$ of $-35 \mathrm{~dB}$ and $-20 \mathrm{~dB}$, respectively, and the -10 $\mathrm{dB}$ bandwidth goes from 1 to $1.2 \mathrm{GHz}$ with the presence of the metamaterial at issue. Therefore, the presence of the absorber improved the performance of the antenna in terms of the reflection coefficient and bandwidth. 


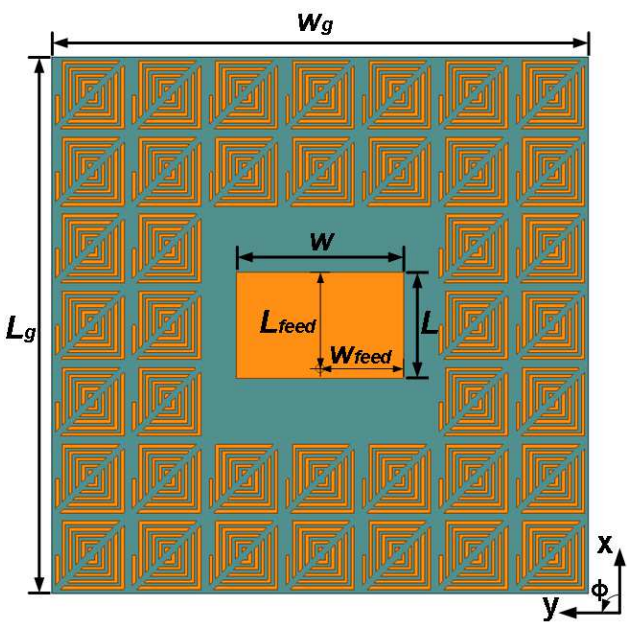

(a)

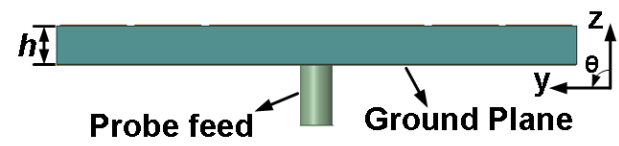

(b)

Figure 4.22: Top (a) and side (b) views of the microstrip antenna with the metamaterial absorbers.

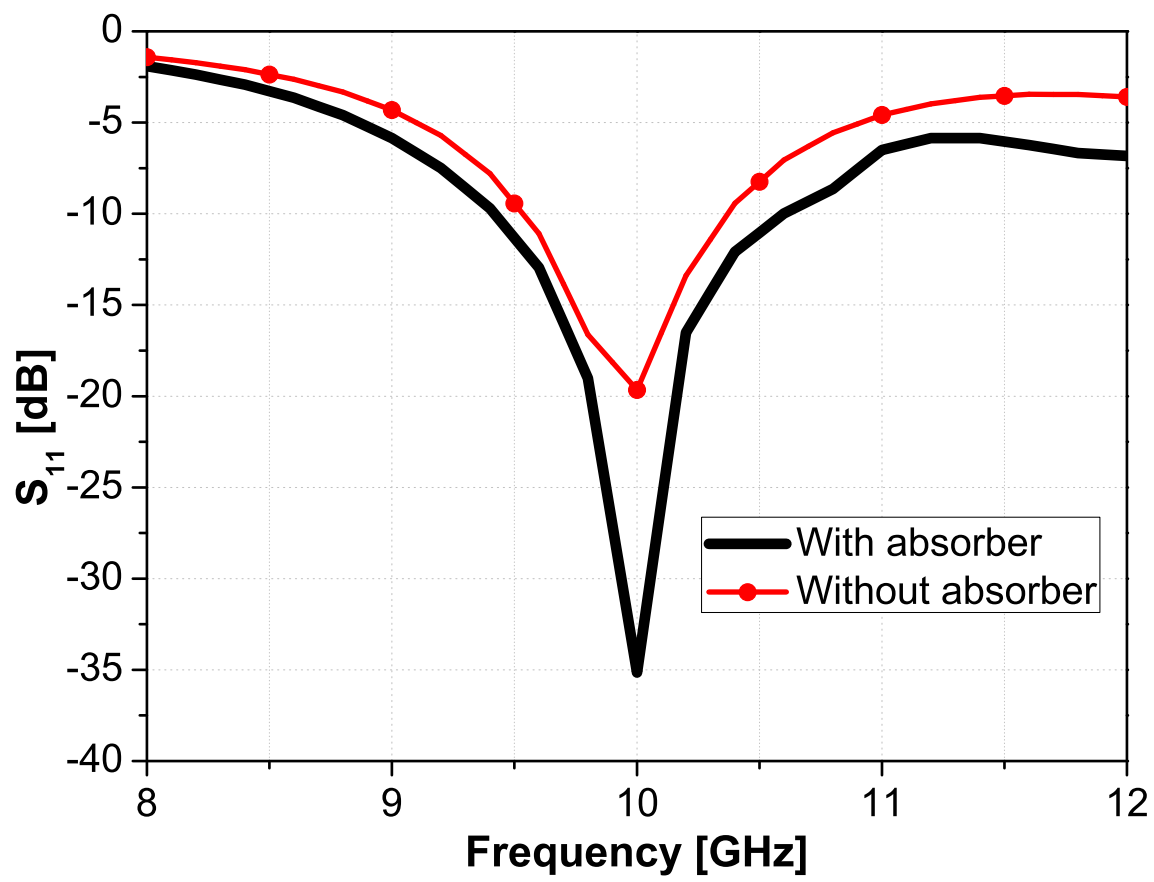

Figure 4.23: Simulated reflection coefficient of the antenna with and without the absorber.

Besides the reflection coefficient, it is important to analyze the radiation pattern of the antennas. Figure 4.24 shows the radiation pattern at $10 \mathrm{GHz}$ for the E- and H-planes. It is possible to note a gain reduction with the presence of the metamaterial absorber. To be more specific, the peak gains are $1.29 \mathrm{dBi}$ and $5.68 \mathrm{dBi}$ for the microstrip antennas with and without the absorber.

Figure 4.25 shows the bistatic RCS at $10 \mathrm{GHz}$ for both yoz and xoz planes considering a $\theta$-polarized incident plane wave. For the yoz plane, it is 


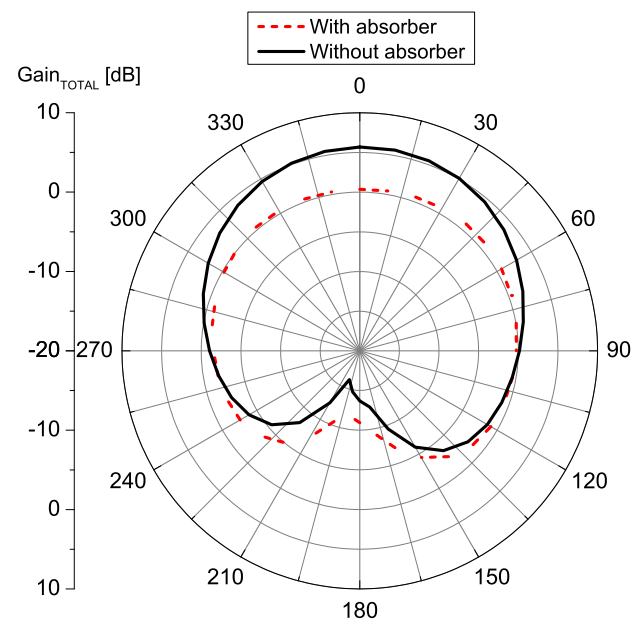

(a)

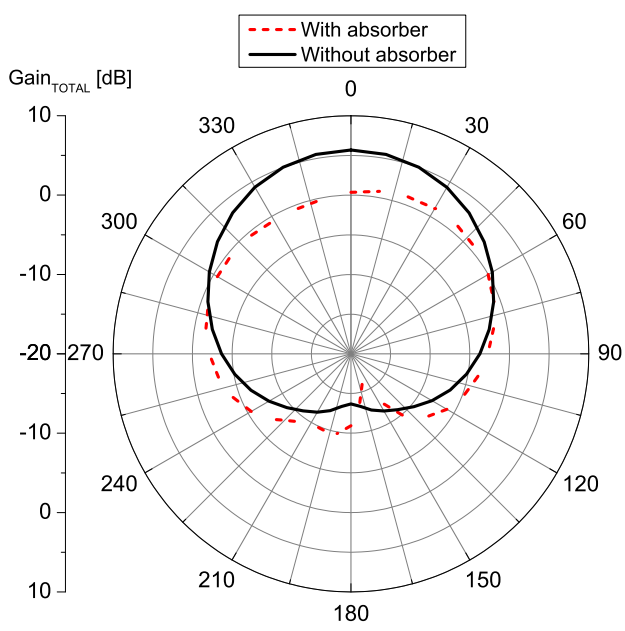

(b)

Figure 4.24: Simulated radiation pattern of the patch antenna at $10 \mathrm{GHz}$ of the E-plane (a) and H-plane (b).

possible to observe reductions of the RCS up to $5.8 \mathrm{~dB}$ from $-90^{\circ}$ to $+79.2^{\circ}$, respectively. For the xoz plane, the absorbers provide RCS reductions up to $8.26 \mathrm{~dB}$ from $-53.83^{\circ}$ to $+54.00^{\circ}$. Therefore, the presence of the proposed metamaterial absorber around the patch can provide a great reduction in the RCS.

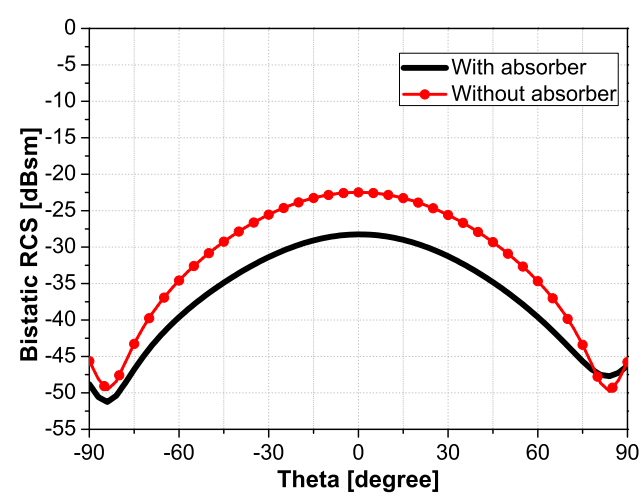

(a)

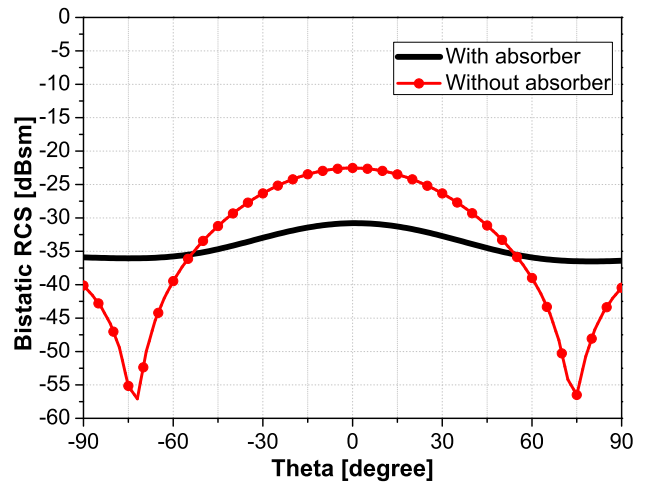

(b)

Figure 4.25: Simulated bistatic RCS of the antenna with and without absorber at $10 \mathrm{GHz}$ at the plane yoz (a) and xoz (b).

\subsubsection{2}

\section{RCS Analysis of a Microstrip Antenna Array}

In order to provide a deeper insight into the influence of the proposed metamaterial absorber on antennas, it is considered a $1 \times 2$ microstrip antenna array operating at $10 \mathrm{GHz}$, as can be observed in Figure 4.26. The dimensions of the absorber are the same ones considered on the previous subsection for the 
microstrip patch antenna. Furthermore, the dimensions of the patch array, in $\mathrm{mm}$, are $h=1.9, W_{g}=51.17, L_{g}=25.585, W=7.93, L=5.07, L_{\text {feed }}=4.85$, and $W_{\text {feed }}=W / 2$ and the Rogers 6006 substrate is employed as well. The center-to-center distance between the patches is $25.585 \mathrm{~mm}$ or, in terms of the operating wavelength, $0.85 \lambda_{0}$.

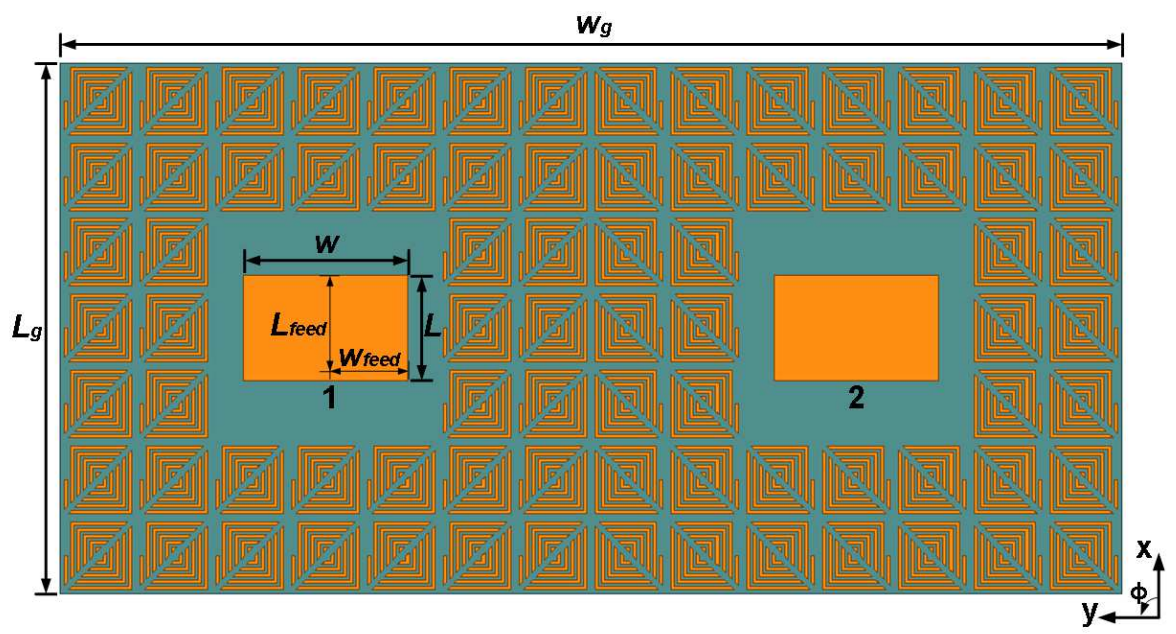

Figure 4.26: Top view of the proposed microstrip patch antenna array.

Figure 4.27 shows the reflection coefficient in port 1 for both microstrip array with and without the absorbers. It is possible to note that there is no shift in the operating frequency and there is an improvement in the reflection coefficient with the presence of the absorbers. To be more specific, the reflection coefficients of the port 1 of the array with and without the absorber at $10 \mathrm{GHz}$ are -31.36 and $-22.84 \mathrm{~dB}$, respectively. Also, once the considered antenna arrays present a two-fold symmetry, the S-parameters of the port 1 and 2 are equal.

The radiation patterns of the array at the E- and H-planes were plotted as well, as can be observed in Figure 4.28. It is important to point out that these radiation patterns were calculated with both ports receiving the same amount of power and in-phase. Furthermore, the peak gains are 2.63 and $9.10 \mathrm{dBi}$ for the array with and without the absorber, respectively. The presence of the absorbers provided a reduction in the peak gain. But, in theory, this reduction can be minimized by either increasing the distance between the absorbers and the patch or decreasing the number of absorber elements.

The bistatic RCS plots, as a function of $\theta$, of the proposed antenna arrays with and without the absorbers are shown in Figure 4.29. At the yoz plane, RCS reductions up to $12.8 \mathrm{~dB}$ are noted from $-54.00^{\circ}$ to $57.00^{\circ}$. Moreover, at the xoz plane, from $-62.15^{\circ}$ to $-51.00^{\circ}$, reductions up to $7.9 \mathrm{~dB}$ are observed. 


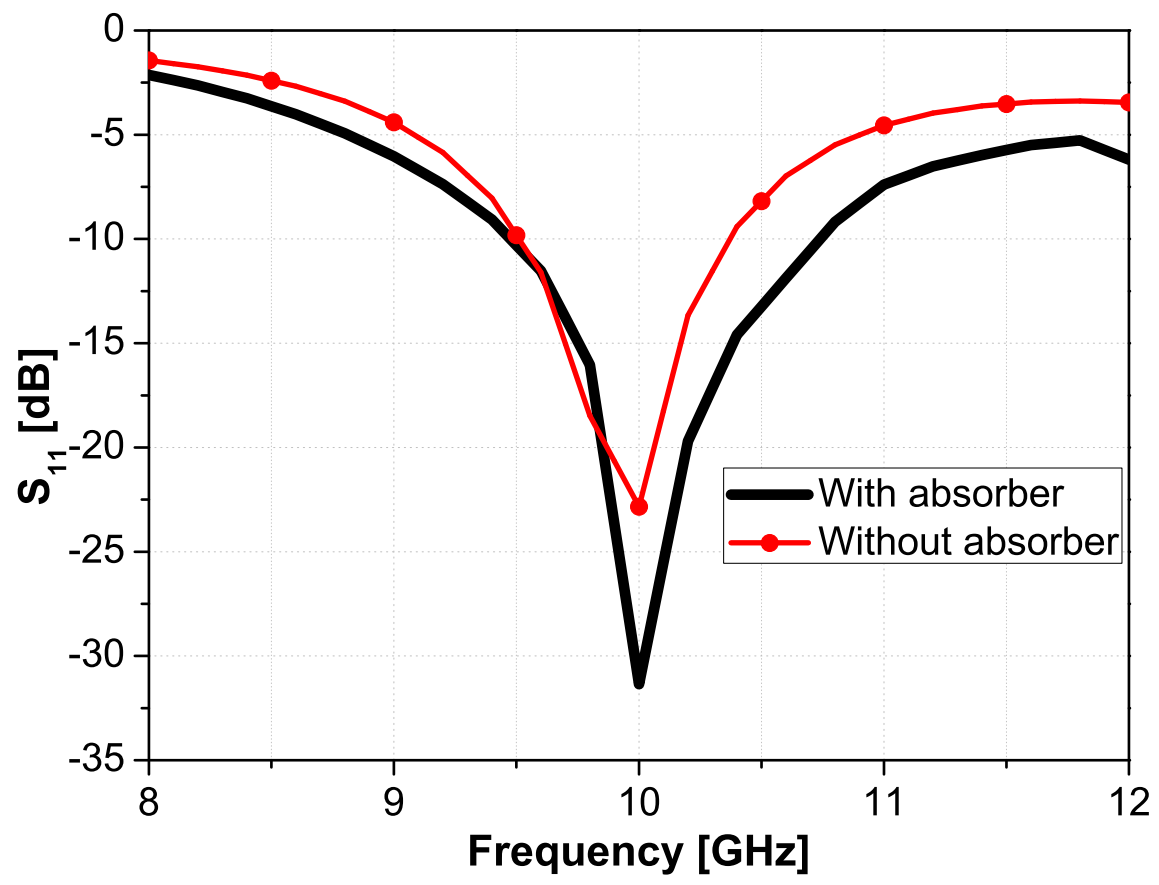

Figure 4.27: Simulated reflection coefficient of the proposed antenna array with and without the absorber.

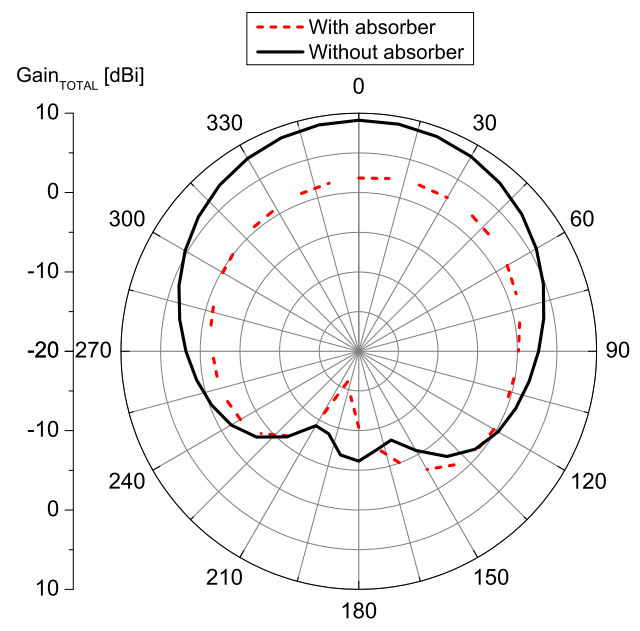

(a)

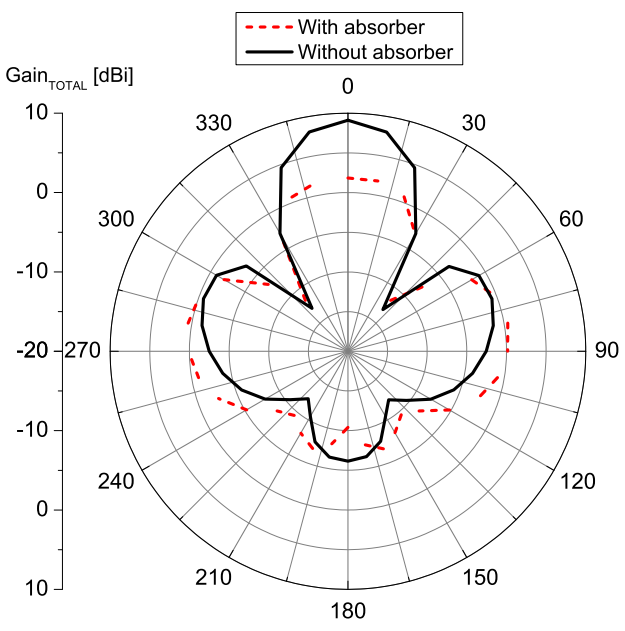

(b)

Figure 4.28: Simulated radiation pattern of the microstrip antenna array at 10 $\mathrm{GHz}$ of the E-plane (a) and H-plane (b).

Therefore, the employment of the proposed absorber is useful and efficient for the RCS reduction.

When it comes to antenna arrays, it is important to investigate the transmission coefficient between the antenna elements. Taking this into account, the transmission coefficients $S_{21}$ of the arrays are showed in Figure 4.30. At $10 \mathrm{GHz}$, the value of $S_{21}$ is the same for both considered arrays, $-27.6 \mathrm{~dB}$, showing that the presence of the absorber does not influence the mutual coupling between 


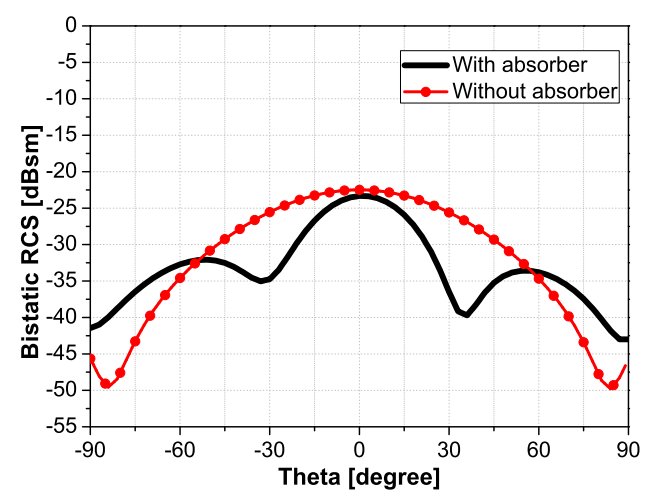

(a)

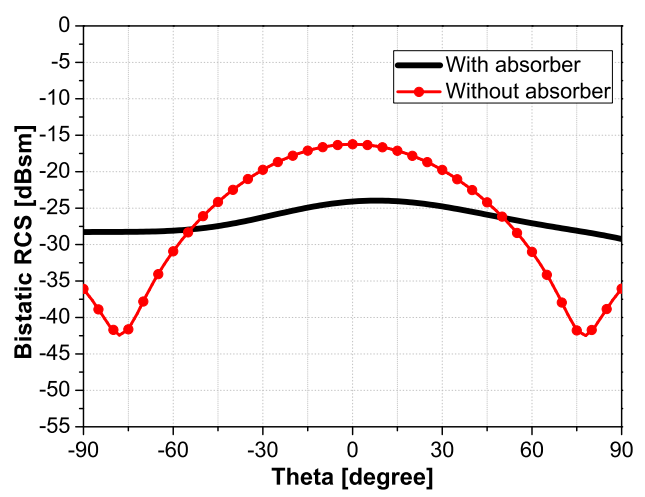

(b)

Figure 4.29: Simulated bistatic RCS of the proposed antenna arrays with and without absorber at $10 \mathrm{GHz}$ at the plane yoz (a) and xoz (b).

the antenna elements of the proposed design. However, it is important to point out that the patches are almost $\lambda_{0}$ away from each other, which is not the ideal scenario to investigate whether the absorber is relevant to the mutual coupling or not. So, to better understand the effects of the proposed absorber on the mutual coupling, an antenna array with spatially close elements is considered in the following.

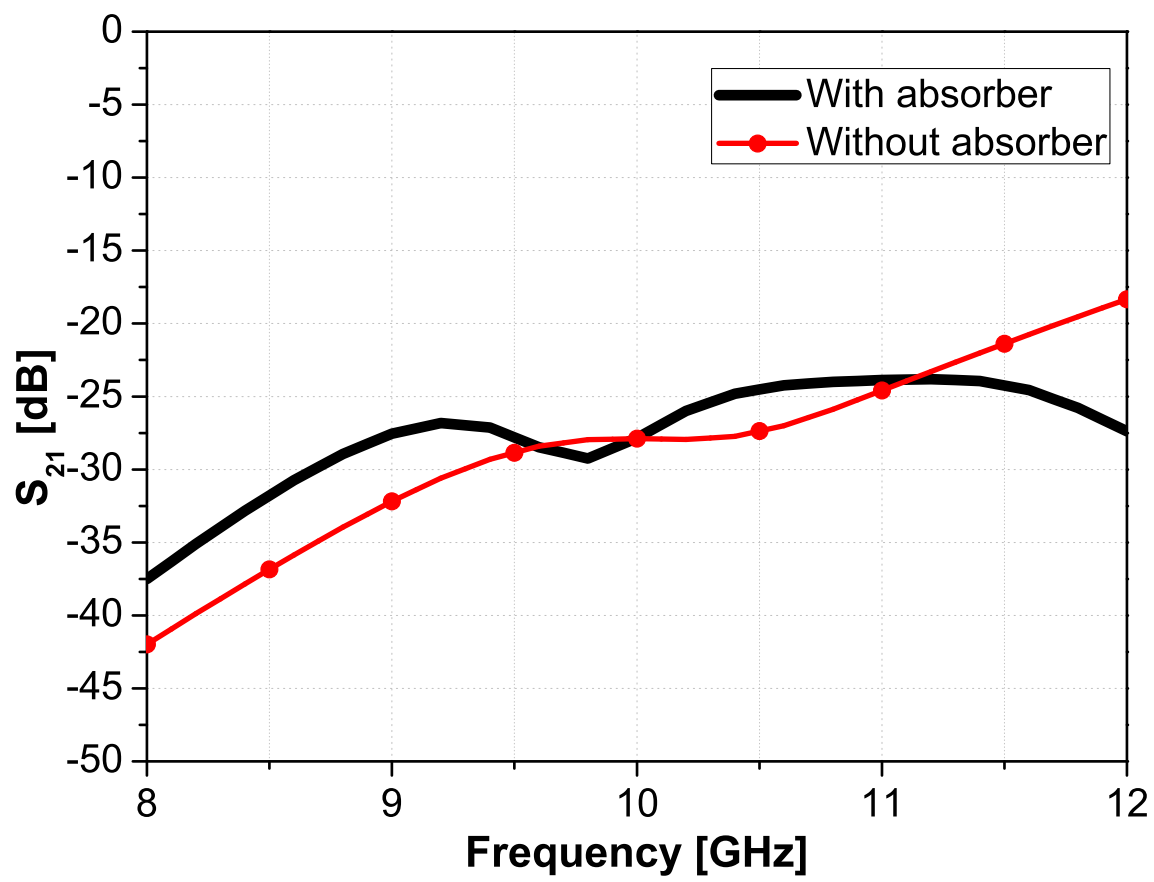

Figure 4.30: Simulated $S_{21}$ of the proposed antenna arrays with and without the absorber. 


\subsection{2}

\section{Mutual Coupling Reduction on a Spatially Close Microstrip Array}

The multiple-input multiple-output (MIMO) antennas have been deeply explored due to their potential to fulfill some of the most important requirements of the modern wireless communication systems, which are higher data rate, shorter latency, and reduction in fading effects, to name a few [73], [74]. However, the mutual coupling between inter-elements of a MIMO antenna is able to produce several drawbacks, such as the degradation of the radiation features and low carrier to noise ratio, for instance [75], which is caused by the signal leakage via conducting currents on the metallic background or surface wave along substrates [76]. To attenuate the mutual coupling in arrays, electromagnetic band-gap (EBG) [77], [78] and defected ground structures (DGS) [79], [80] have been efficiently used. However, these structures present some drawbacks. The occupied space by the EBG structures and the backward signal leakage caused by the DGSs limit their employment [76].

In this work, a metamaterial absorber wall is proposed to reduce the mutual coupling between antenna elements. The structure is designed to operate at the $3.4-3.8 \mathrm{GHz}$ band, which is one of the most explored frequency range for the fifth-generation (5G) technology [81]. Moreover, the design of the employed absorber is the same one proposed in this Chapter, as in Figure 4.4 , and its dimensions, in $\mathrm{mm}$, are $a=5.4, w=0.2, p=0.1, g=0.2$, and $d=0.2$. The thickness of the absorber is $3.8 \mathrm{~mm}$ and its substrate is the Rogers $5880\left(\epsilon_{r}=2.2\right)$. Considering these dimensions and material, the proposed absorber presents absorption above $90 \%$ from 3.25 to $4.70 \mathrm{GHz}$, covering the aimed frequency band, as can be seen in Figure 4.31. Therefore, the fractional bandwidth of the proposed absorber is $36.47 \%$.

To verify the efficiency of the proposed absorber to reduce the mutual coupling, a $1 \times 2$ microstrip array is designed to operate at the $3.4-3.8$ $\mathrm{GHz}$ band, as can be observed in Figure 4.32. The proposed structure presents two inset-fed patches and one absorber wall between them. Moreover, it is considered two $3 \times 6$ matrix of absorber unit cells in order to provide a twofold symmetry along the $\mathrm{x}$-axis, which simplifies the analysis of the structure. The feeding microstrip line is designed to present $50 \Omega$ of impedance.

The dimensions of the proposed array, in $\mathrm{mm}$, are $h=3.8, L_{g}=32.4$, $W_{g}=83.28, W=17.61, L=11.1, w_{0}=1.83, y_{0}=3.4, L_{50}=15.6$, $W_{50}=3.65, h_{1}=3.8, h_{2}=16.2, d_{1}=8.22$, and $d_{2}=41.64$, and the substrate 1 is the Rogers 6006. Considering these values, the S-parameters are calculated under two scenarios, as can be seen in 4.33, which are with and without the decoupling wall. One can note that the presence of absorber provides an upward 


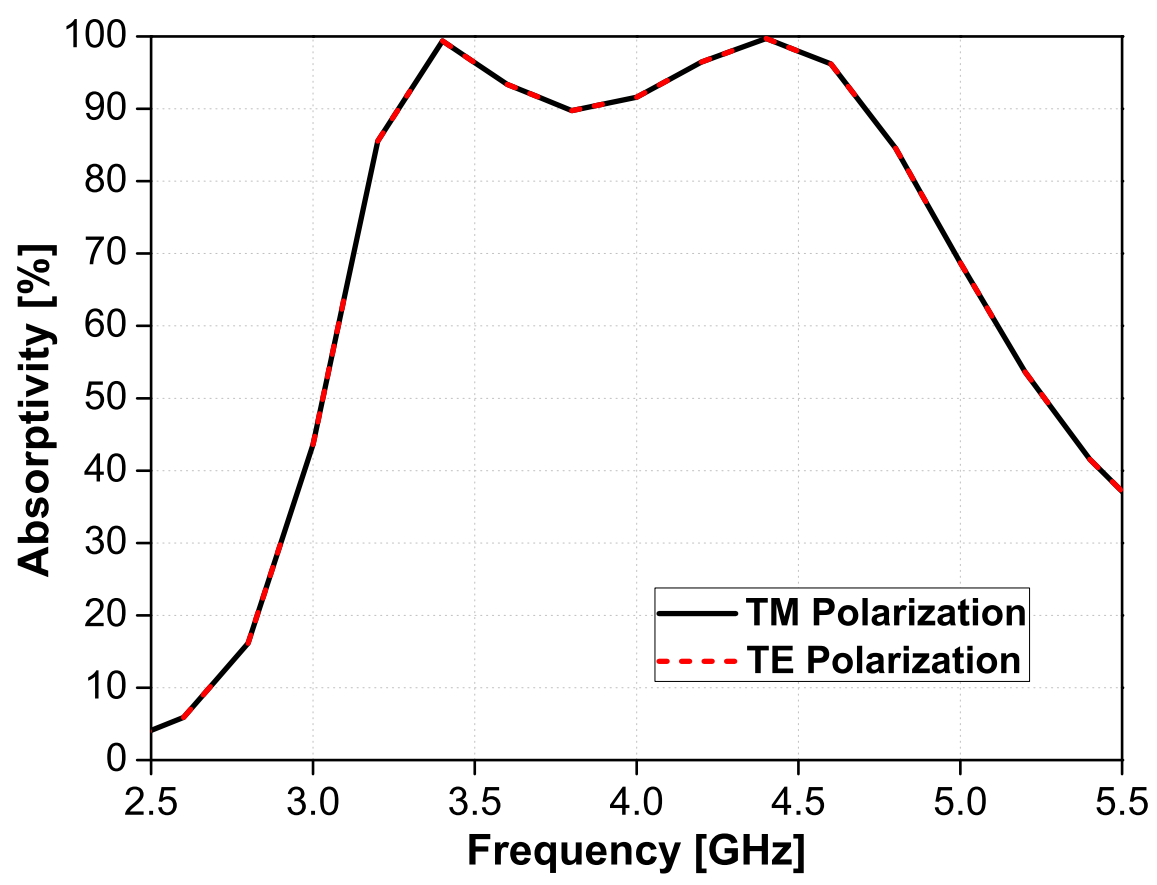

Figure 4.31: Absorptivity of the proposed absorber for 5G applications.

shift in the operating frequency from 3.6 to $3.7 \mathrm{GHz}$. However, the $S_{11}$ is -22 $\mathrm{dB}$ and $-12 \mathrm{~dB}$ at the respective central frequencies of the array with and without the absorber wall, and, thus, the reflection coefficient is better with the presence of the metamaterial. Furthermore, the mutual coupling between the antenna elements decreases from $-19 \mathrm{~dB}$ to $-34 \mathrm{~dB}$ with the employment of the absorbers, showing a relevant improvement of this factor considering a relatively small distance between the patches, which is $\sim \frac{\lambda_{0}}{2}$.

In order to illustrate the efficiency of the absorber wall to suppress the mutual coupling, the surface current densities at the respective operating frequencies of the microstrip arrays with and without metamaterials are plotted, as can be seen in Figure 4.34, considering that the port 2 is terminated with a $50 \Omega$ load, while the port 1 is excited. So, it is possible to note that the surface current density on the patch 2 is considerably reduced with the presence of the absorber wall, confirming the results of the S-parameters. Furthermore, Figure 4.35 shows the perspective view of the surface current density of the array with the absorber wall. It is possible to observe that there is a high concentration of surface current on the absorbers, as expected.

Besides the S-parameters, it is important to investigate some other parameters of the antenna. Taking this into consideration, the radiation patterns at the E-plane and H-plane in the presence and absence of the metamaterial are shown in Figure 4.36. There are no bigger changes in the shape of the radiation pattern at the planes at issue. Furthermore, the peak 


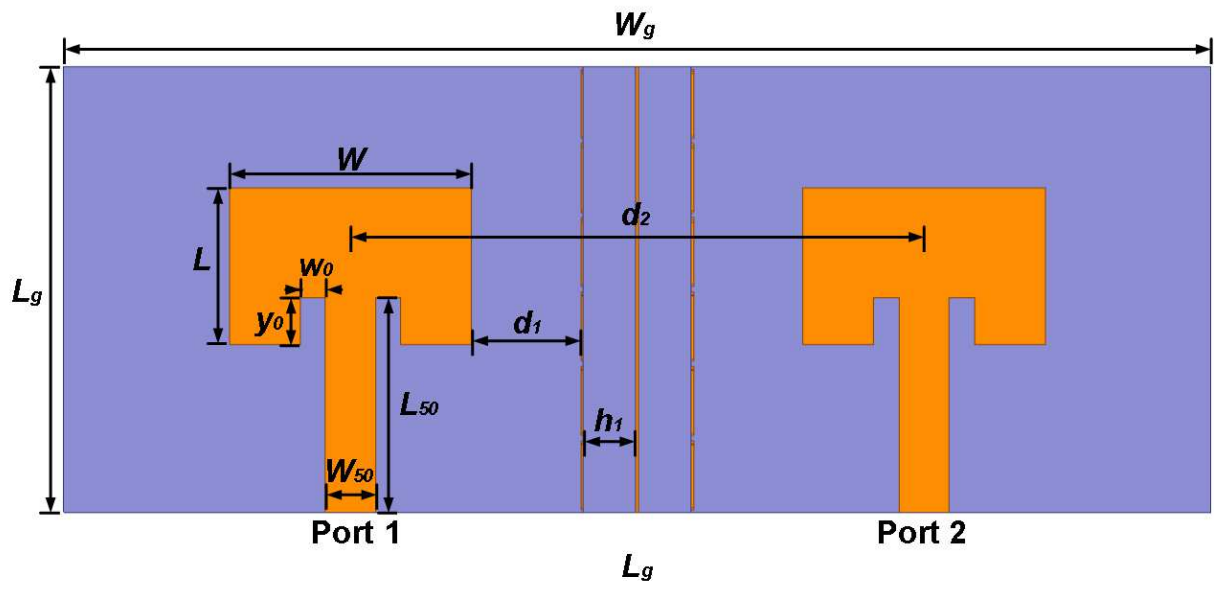

(a)

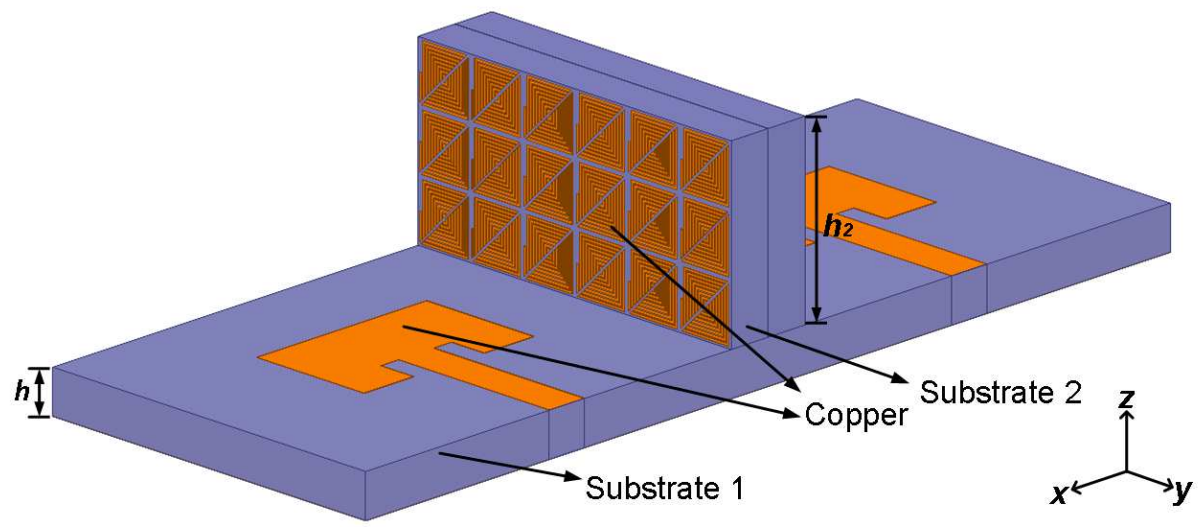

(b)

Figure 4.32: Top (a) and perspective (b) views of the microstrip antenna array with the metamaterial absorber wall.

gains are 4.56 and $5.79 \mathrm{dBi}$ for the array with and without the metamaterial wall, respectively. Therefore, the decrease of approximately $1 \mathrm{~dB}$ in the peak gain sounds like an irrelevant price to pay for a great improvement in the mutual coupling between the inter-elements of the proposed array.

The employment of the metamaterial absorber to reduce the mutual coupling presented some promising results, once the isolation between the elements was drastically improved. Additionally, the presence of the decoupling wall does not compromise the performance of the array even considering a relatively small distance between the antenna elements.

\section{5}

\section{Experimental Results}

To perform the experimental measurements of the proposed absorber, a sample of $110 \mathrm{~mm} \times 110 \mathrm{~mm} \times 1.6 \mathrm{~mm}$, or $31 \times 31$ unit cells, was manufactured using an FR-4 substrate and considering the dimensions of Model 1, as in Table 


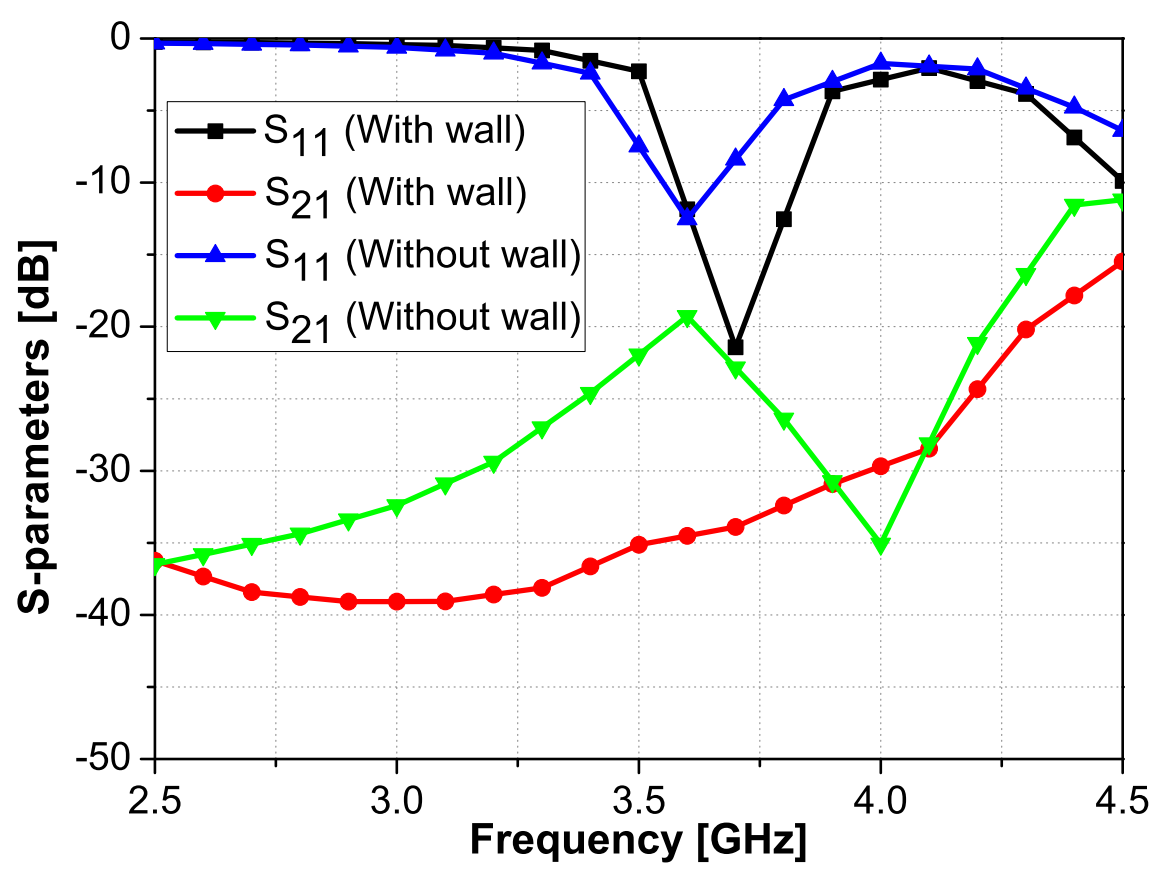

Figure 4.33: Simulated S-parameters of the array with and without the absorber wall.
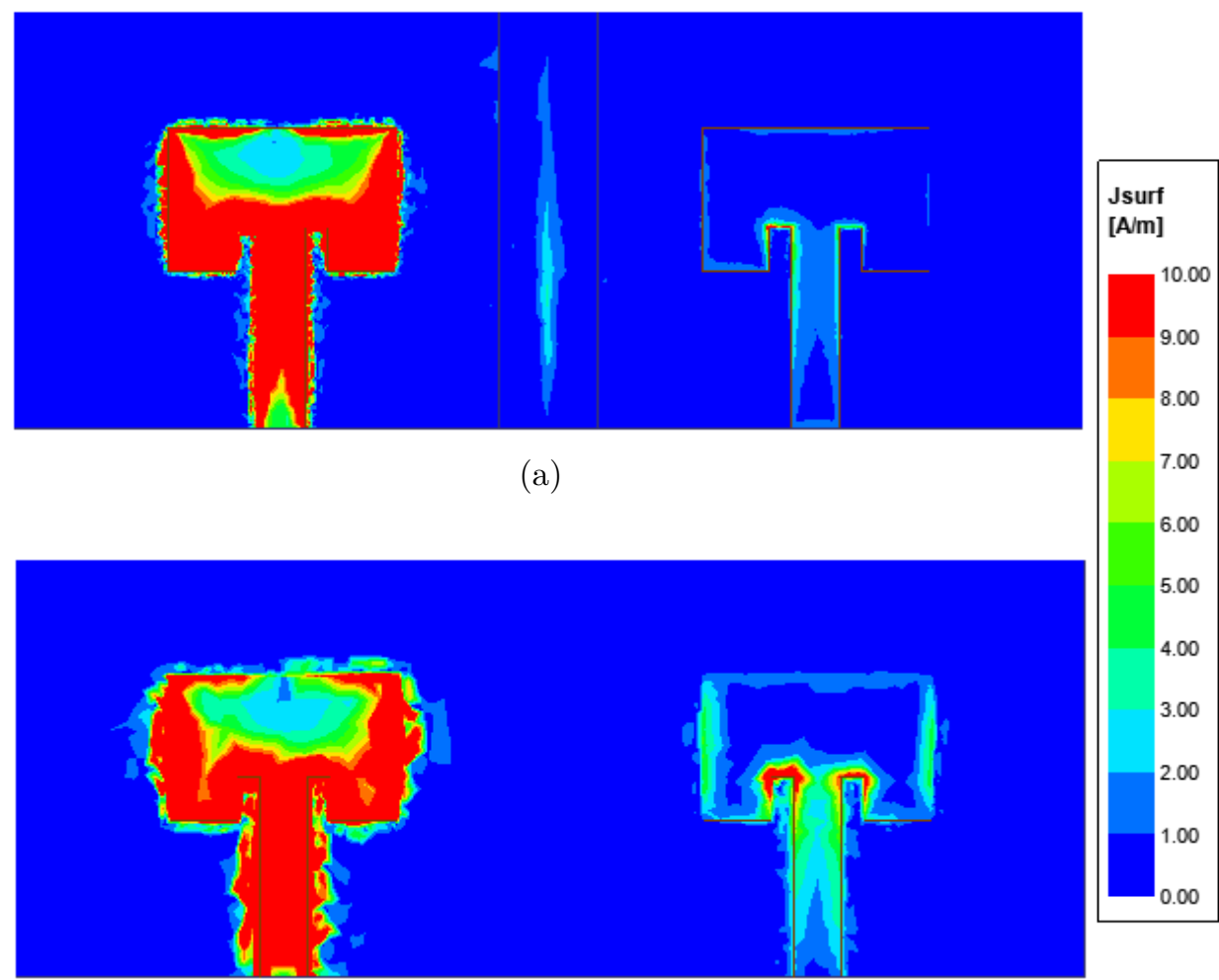

(b)

Figure 4.34: Top view of the surface current density at 3.7 (a) and 3.6 (b) GHz for the arrays with and without the absorber wall, respectively. 


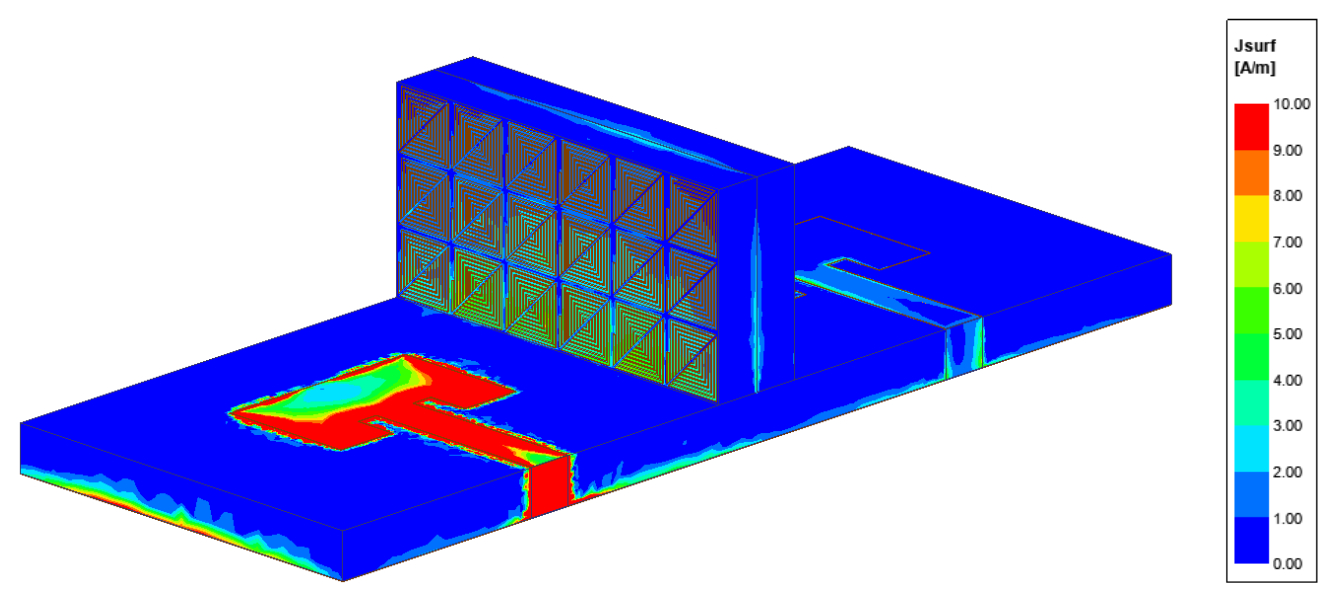

(a)

Figure 4.35: Perspective view of the surface current density on the microstrip antenna array with the decoupling wall at $3.7 \mathrm{GHz}$.

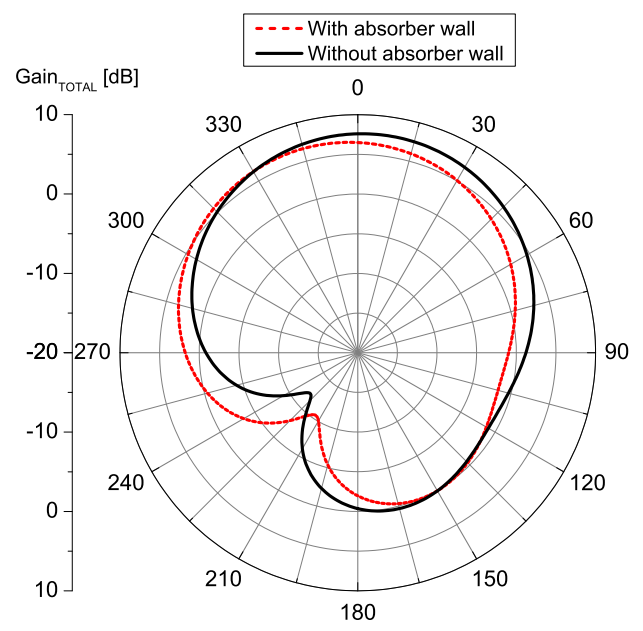

(a)

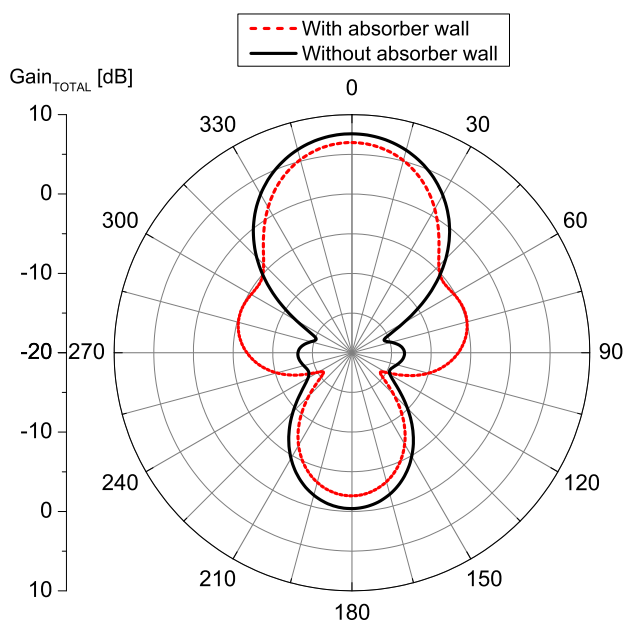

(b)

Figure 4.36: Simulated radiation pattern of E-plane (a) and H-plane (b) at 3.6 and $3.7 \mathrm{GHz}$ for the microstrip array with and without the absorber wall, respectively.

4.1. Furthermore, photographs of the metamaterial absorber sample are shown in Figure 4.37. The length of the sample at issue is, in terms of the wavelength at $11.44 \mathrm{GHz}$, is $4.19 \lambda_{L}$. Therefore, the sample is larger enough to fulfill the size constraint for a free-space measurement, which minimizes the effects of scattering at the boundaries of the absorber [33].

The sample of the proposed absorber was measured at the DLR (German Aerospace Center or, in German, Deutsches Zentrum für Luft- und Raumfahrt) laboratories. A Ku band (12-18 GHz) setup was used during the measurements, as shown in Figure 4.38. Also, a vector network analyzer ANRITSU VectorStar MS4644B, a circular horn, and a dielectric lens were employed to measure the 


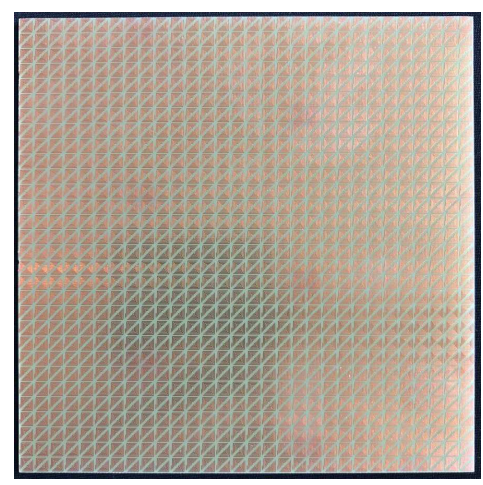

(a)

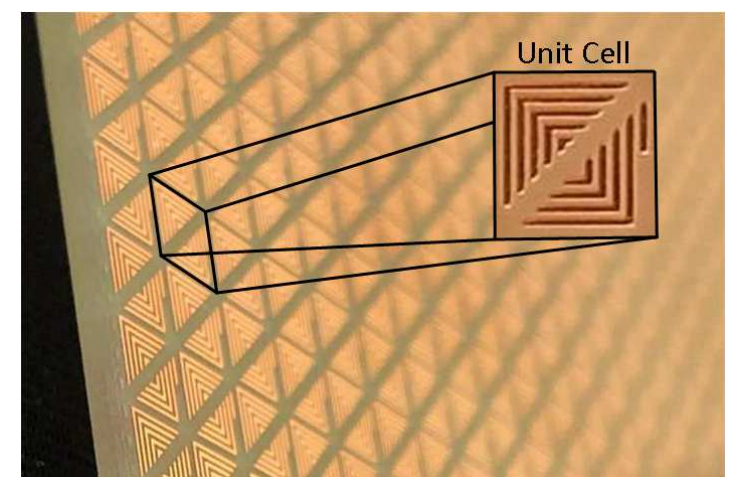

(b)

Figure 4.37: Photograph of the fabricated metamaterial absorber proposed: top (a) and perspective (b) views.

reflection of the sample under normal and oblique incidences. It is important to point out that the measurements were carried out in an anechoic chamber.

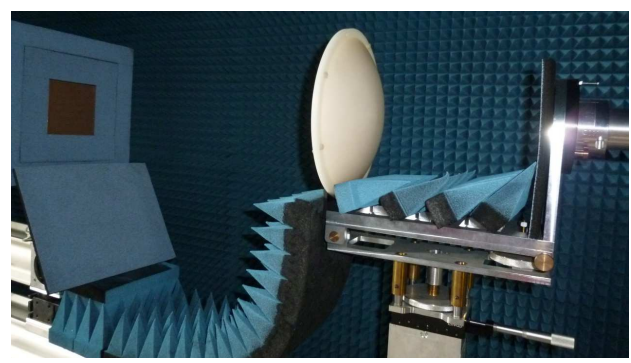

(a)

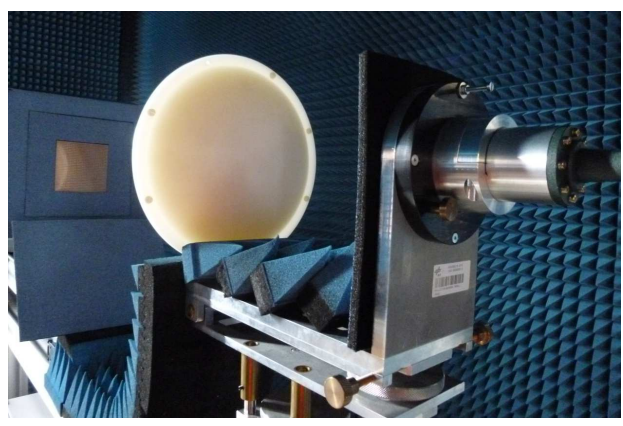

(b)

Figure 4.38: Photograph of the setup of measurements at DLR.

Figure 4.39 shows the measured and simulated curves of absorptivity of the proposed metamaterial absorber under normal incidence at the $\mathrm{Ku}$ band. One can notice that the measured absorptivity shows a high level of absorption over the frequency band at issue and good matching with the simulated curve. Furthermore, the minor difference between the curves is attributed to the finite size of the sample and the manufacturing tolerance.

In order to provide an understanding of the behavior of the sample under oblique incidence, measurements were performed at 14.5, 15.0 and $15.5 \mathrm{GHz}$ for TE as well as TM polarization for different incidence angles. Figure 4.40 shows the curve of absorptivity against the incidence angle, which ranges from $0^{\circ}$ to $20^{\circ}$ with steps of $0.5^{\circ}$. It is possible to observe that the behavior is almost the same over the considered angle range for both TE and TM polarization. Furthermore, the metamaterial sample presented almost $100 \%$ of absorption at the three frequencies at issue, demonstrating great angle stability. One can 


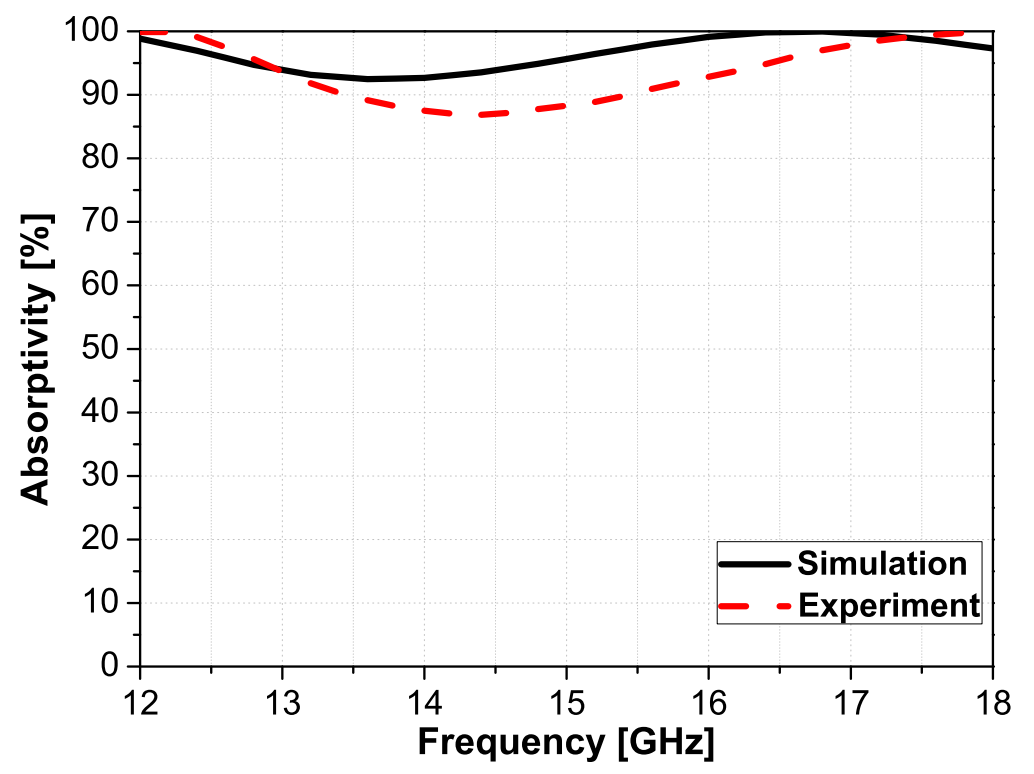

Figure 4.39: Simulated and measured absorptivity of the proposed absorber under normal incidence.

notice that there is a slight deviation in the curves of absorptivity around $18^{\circ}$, which does not compromise the performance of the absorber, once, in the worst case, the absorptivity remains above $80 \%$.

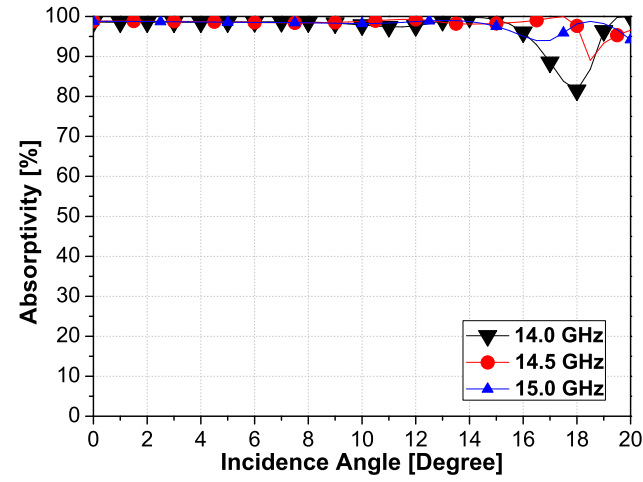

(a)

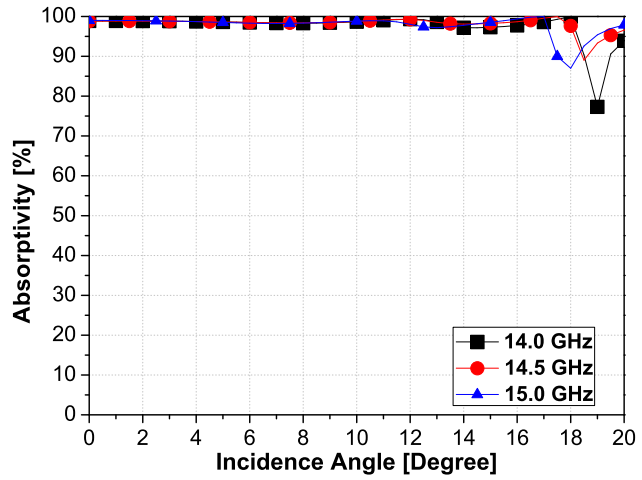

(b)

Figure 4.40: Absorptivity per incidence angle plot for both (a) TE and (b) TM polarization.

Therefore, the experimental measurement validated the proposed metamaterial absorber. The experiments under normal and oblique incidences demonstrated a good agreement with the simulated results. 


\section{5}

\section{Conclusion and Future Work}

In this present work, a compact ultrathin ultrawideband metamaterial absorber based on square spirals was proposed. In addition, an algorithm based on the least square method was introduced to describe a broadband absorber as an RLC circuit.

The efficiency and flexibility of the proposed metamaterial absorber were demonstrated by showing a variety of results from the electromagnetic simulator with different parameters and materials. One of the most important simulated results showed a bandwidth of absorption above $90 \%$ from 11.44 to 20.00 using an FR-4 substrate, considering a single-layer structure with a sub-wavelength unit cell. Moreover, the proposed metamaterial demonstrated angle stability up to $40^{\circ}$ and $50^{\circ}$ for the $\mathrm{TE}$ and $\mathrm{TM}$ polarization, respectively. The absorptivity under normal incidence is the same for TE as well as TM polarization, which means that the structure is polarization insensitive.

The employment of the proposed absorber in antennas was investigated as well. At first, a probe-fed microstrip antenna operating at $10 \mathrm{GHz}$ was introduced and two rows of absorbers were placed around the patch, which were designed to present absorption above $90 \%$ over the $\mathrm{X}$ band. It was observed that the presence of the absorber provided RCS reductions up to 8.26 dB. Furthermore, it was considered a $1 \times 2$ microstrip array with the proposed absorber. Considering this structure, RCS reductions up to $12.8 \mathrm{~dB}$ were found. Therefore, the proposed metamaterial is effective in RCS reduction of microstrip antennas.

To describe a broadband absorber as an equivalent circuit, a multiresonant circuit was proposed and a new algorithm was introduced to extract the values of the lumped elements. The curve of absorptivity of the equivalent circuit presented a good agreement with the result obtained in the EM simulation. It is important to point out that the algorithm and the equivalent circuit are presented with a straightforward approach and it can be employed in other broadband absorber structures.

A metamaterial wall based on the proposed absorber is introduced to reduce the mutual coupling between the antenna elements of a microstrip array. In addition, the structure is designed to operate at the $3.4-3.8 \mathrm{GHz}$ 
frequency range, which is a potential band for the $5 \mathrm{G}$ applications, and the distance between the patches are $\approx \frac{\lambda_{0}}{2}$. Reductions in the mutual coupling up to $15 \mathrm{~dB}$ were found with the presence of the absorber wall, showing the effectiveness of the proposed structures in the isolation between the antenna elements.

Finally, to experimentally verify the performance of the proposed absorber, a sample of $110 \mathrm{~mm} \times 110 \mathrm{~mm} \times 1.6 \mathrm{~mm}$ was manufactured using an FR-4 dielectric substrate. The measurements were carried out in a $\mathrm{Ku}$ band setup considering both normal and oblique incidence. The results of absorptivity of the sample under normal incidence presented broadband of absorption over the frequency band at issue and showed a good matching with the absorptivity from the full-wave analysis. Moreover, the sample was tested under oblique incidence at 14.5, 15.0, and $15.5 \mathrm{GHz}$, with the incidence angle ranging from $0^{\circ}$ to $20^{\circ}$ considering both $\mathrm{TE}$ and $\mathrm{TM}$ polarization. The results demonstrated great angle stability over the incidence angle analyzed, showing a level of absorption of almost $100 \%$ over the angle range at issue for both orthogonal polarization considered.

As a suggestion of future work, we plan to explore different types of metal with higher resistivity than the copper in order to broaden even more the bandwidth of absorption. Furthermore, the design and characterization of multilayer absorbers based on square spirals is a promising possibility of future development of this work as well as the experimental measurements on the applications of the absorbers, such as the reduction on the mutual coupling and improvements on the radar cross-section of antennas and arrays. 


\section{Bibliography}

[1] W. Emerson, "Electromagnetic wave absorbers and anechoic chambers through the years," IEEE Transactions on Antennas and Propagation, vol. 21, no. 4, pp. 484-490, 1973.

[2] T. Maier and H. Brückl, "Wavelength-tunable microbolometers with metamaterial absorbers," Optics letters, vol. 34, no. 19, pp. 3012-3014, 2009.

[3] H. Wang, V. P. Sivan, A. Mitchell, G. Rosengarten, P. Phelan, and L. Wang, "Highly efficient selective metamaterial absorber for high-temperature solar thermal energy harvesting," Solar Energy Materials and Solar Cells, vol. 137, pp. 235-242, 2015.

[4] X. Liu, T. Tyler, T. Starr, A. F. Starr, N. M. Jokerst, and W. J. Padilla, "Taming the blackbody with infrared metamaterials as selective thermal emitters," Physical review letters, vol. 107, no. 4, p. 045901, 2011.

[5] H. Wang, J.-Y. Chang, Y. Yang, and L. Wang, "Performance analysis of solar thermophotovoltaic conversion enhanced by selective metamaterial absorbers and emitters," International Journal of Heat and Mass Transfer, vol. 98, pp. 788-798, 2016.

[6] F. Dincer, O. Akgol, M. Karaaslan, E. Unal, and C. Sabah, "Polarization angle independent perfect metamaterial absorbers for solar cell applications in the microwave, infrared, and visible regime," Progress In Electromagnetics Research, vol. 144, pp. 93-101, 2014.

[7] A. E. Culhaoglu, A. V. Osipov, and P. Russer, "Mono-and bistatic scattering reduction by a metamaterial low reflection coating," IEEE Transactions on Antennas and Propagation, vol. 61, no. 1, pp. 462-466, 2013.

[8] R. Ling, J. Scholler, and P. Y. Ufimtsev, "The propagation and excitation of surface waves in an absorbing layer," Progress In Electromagnetics Research, vol. 19, pp. 49-91, 1998.

[9] N. I. Landy, S. Sajuyigbe, J. J. Mock, D. R. Smith, and W. J. Padilla, "Perfect metamaterial absorber," Physical review letters, vol. 100, no. 20, p. 207402, 2008. 
[10] N. Engheta and R. W. Ziolkowski, Metamaterials: physics and engineering explorations. John Wiley \& Sons, 2006.

[11] F. Costa, A. Monorchio, and G. Manara, "Efficient analysis of frequencyselective surfaces by a simple equivalent-circuit model," IEEE Antennas and Propagation Magazine, vol. 54, no. 4, pp. 35-48, 2012.

[12] S. Ghosh, S. Bhattacharyya, Y. Kaiprath, and K. Vaibhav Srivastava, "Bandwidth-enhanced polarization-insensitive microwave metamaterial absorber and its equivalent circuit model," Journal of Applied Physics, vol. 115, no. 10, p. 104503, 2014.

[13] S. Bhattacharyya, S. Ghosh, and K. V. Srivastava, "Equivalent circuit model of an ultra-thin polarization-independent triple band metamaterial absorber," AIP Advances, vol. 4, no. 9, p. 097127, 2014.

[14] J. C. Maxwell, A treatise on electricity and magnetism, vol. 1. Clarendon press, 1881.

[15] C. A. Balanis, Advanced engineering electromagnetics. John Wiley \& Sons, 1999.

[16] S. J. Orfanidis, "Electromagnetic waves and antennas," 2002.

[17] D. M. Pozar, "Microwave engineering," Wiley, 2012.

[18] A. Lai, K. M. Leong, and T. Itoh, "Infinite wavelength resonant antennas with monopolar radiation pattern based on periodic structures," IEEE transactions on antennas and propagation, vol. 55, no. 3, pp. 868-876, 2007.

[19] B. Shen, P. Wang, R. Polson, and R. Menon, "Ultra-high-efficiency metamaterial polarizer," Optica, vol. 1, no. 5, pp. 356-360, 2014.

[20] N. Fang, H. Lee, C. Sun, and X. Zhang, "Sub-diffraction-limited optical imaging with a silver superlens," Science, vol. 308, no. 5721, pp. 534-537, 2005.

[21] V. G. Veselago, "The electrodynamics of substances with simultaneously negative values of and $\mu$," Soviet physics uspekhi, vol. 10, no. 4, p. 509, 1968.

[22] R. Marques, F. Martin, and M. Sorolla, Metamaterials with negative parameters: theory, design, and microwave applications, vol. 183. John Wiley \& Sons, 2011. 
[23] J. B. Pendry, A. J. Holden, D. J. Robbins, and W. Stewart, "Magnetism from conductors and enhanced nonlinear phenomena," IEEE transactions on microwave theory and techniques, vol. 47, no. 11, pp. 2075-2084, 1999.

[24] R. A. Shelby, D. R. Smith, and S. Schultz, "Experimental verification of a negative index of refraction," science, vol. 292, no. 5514, pp. 77-79, 2001.

[25] G. Dolling, M. Wegener, S. Linden, and C. Hormann, "Photorealistic images of objects in effective negative-index materials," Optics express, vol. 14, no. 5, pp. 1842-1849, 2006.

[26] C. Caloz and T. Itoh, "Novel microwave devices and structures based on the transmission line approach of meta-materials," in Microwave Symposium Digest, 2003 IEEE MTT-S International, vol. 1, pp. 195-198, IEEE, 2003.

[27] C. L. Holloway, E. F. Kuester, J. A. Gordon, J. O'Hara, J. Booth, and D. R. Smith, "An overview of the theory and applications of metasurfaces: The twodimensional equivalents of metamaterials," IEEE Antennas and Propagation Magazine, vol. 54, no. 2, pp. 10-35, 2012.

[28] A. Nicolson and G. Ross, "Measurement of the intrinsic properties of materials by time-domain techniques," IEEE Transactions on instrumentation and measurement, vol. 19, no. 4, pp. 377-382, 1970.

[29] W. B. Weir, "Automatic measurement of complex dielectric constant and permeability at microwave frequencies," Proceedings of the IEEE, vol. 62, no. 1, pp. 33-36, 1974.

[30] C. L. Holloway, A. Dienstfrey, E. F. Kuester, J. F. O'Hara, A. K. Azad, and A. J. Taylor, "A discussion on the interpretation and characterization of metafilms/metasurfaces: The two-dimensional equivalent of metamaterials," Metamaterials, vol. 3, no. 2, pp. 100-112, 2009.

[31] E. F. Kuester, M. A. Mohamed, M. Piket-May, and C. L. Holloway, "Averaged transition conditions for electromagnetic fields at a metafilm," IEEE Transactions on Antennas and Propagation, vol. 51, no. 10, pp. 2641-2651, 2003.

[32] C. L. Holloway, E. F. Kuester, and A. Dienstfrey, "Characterizing metasurfaces/metafilms: The connection between surface susceptibilities and effective material properties," IEEE Antennas and Wireless Propagation Letters, vol. 10, pp. 1507-1511, 2011. 
[33] L.-F. Chen, C. Ong, C. Neo, V. Varadan, and V. K. Varadan, Microwave electronics: measurement and materials characterization. John Wiley \& Sons, 2004.

[34] F. Jay and J. Goetz, "IEEE standard dictionary of electrical and electronics terms," Institute of Electrical and Electronics Engineers, 1988.

[35] D. Jenn, Radar and laser cross section engineering. American Institute of Aeronautics and Astronautics, Inc., 2005.

[36] E. F. Knott, J. F. Schaeffer, and M. T. Tulley, Radar cross section. SciTech Publishing, 2004.

[37] M. I. Skolnik, "Radar handbook," 1970.

[38] A. K. Zadeh and A. Karlsson, "Capacitive circuit method for fast and efficient design of wideband radar absorbers," IEEE Transactions on Antennas and Propagation, vol. 57, no. 8, pp. 2307-2314, 2009.

[39] Comsol, "Modeling of pyramidal absorbers for an anechoic chamber." https://br.comsol.com/model/modeling-of-pyramidal-absorbers-for-ananechoic-chamber-12129. Accessed: 2018-05-30.

[40] Y. Lee, J. Yull Rhee, Y. J. Yoo, and W. Kim, Metamaterials for Perfect Absorption, vol. 236. 012016.

[41] Y.-C. Song, J. Ding, C.-J. Guo, J.-K. Zhang, Y.-H. Ren, and Y. Qu, "A novel mu-negative metamaterial with broad bandwidth," Journal of Modern Optics, vol. 61, no. 20, pp. 1656-1664, 2014.

[42] V. Weston, "Theory of absorbers in scattering," IEEE Transactions on Antennas and Propagation, vol. 11, no. 5, pp. 578-584, 1963.

[43] K. N. Rozanov, "Ultimate thickness to bandwidth ratio of radar absorbers," IEEE Transactions on Antennas and Propagation, vol. 48, no. 8, pp. 12301234, 2000.

[44] F. Costa, A. Monorchio, and G. Manara, "An overview of equivalent circuit modeling techniques of frequency selective surfaces and metasurfaces," Appl. Comput. Electromagn. Soc. J., vol. 29, no. 12, pp. 960-976, 2014.

[45] F. Costa, A. Monorchio, and G. Manara, "Analysis and design of ultra thin electromagnetic absorbers comprising resistively loaded high impedance surfaces," IEEE Transactions on Antennas and Propagation, vol. 58, no. 5, pp. 1551-1558, 2010. 
[46] A. B. Yakovlev, O. Luukkonen, C. R. Simovski, S. A. Tretyakov, S. Paulotto, P. Baccarelli, and G. W. Hanson, "Analytical modeling of surface waves on high impedance surfaces," in Metamaterials and Plasmonics: Fundamentals, Modelling, Applications, pp. 239-254, Springer, 2009.

[47] O. Luukkonen, C. Simovski, G. Granet, G. Goussetis, D. Lioubtchenko, A. V. Raisanen, and S. A. Tretyakov, "Simple and accurate analytical model of planar grids and high-impedance surfaces comprising metal strips or patches," IEEE Transactions on Antennas and Propagation, vol. 56, no. 6, pp. 16241632, 2008.

[48] J. Kong, Electromagnetic Wave Theory. Wiley interscience publication, John Wiley and Sons, 1986.

[49] M. Born and E. Wolf, Principles of optics: electromagnetic theory of propagation, interference and diffraction of light. Elsevier, 2013.

[50] T. Wanghuang, W. Chen, Y. Huang, and G. Wen, "Analysis of metamaterial absorber in normal and oblique incidence by using interference theory," AIP Advances, vol. 3, no. 10, p. 102118, 2013.

[51] D. Q. Vu, D. H. Le, H. T. Dinh, T. G. Trinh, L. Yue, D. T. Le, and D. L. Vu, "Broadening the absorption bandwidth of metamaterial absorber by coupling three dipole resonances," Physica B: Condensed Matter, vol. 532, pp. 90-94, 2018.

[52] S. Li, J. Gao, X. Cao, W. Li, Z. Zhang, and D. Zhang, "Wideband, thin, and polarization-insensitive perfect absorber based the double octagonal rings metamaterials and lumped resistances," Journal of Applied Physics, vol. 116, no. 4 , p. $043710,2014$.

[53] D. Sood and C. C. Tripathi, "A compact ultrathin ultra-wideband metamaterial microwave absorber," Journal of Microwaves, Optoelectronics and Electromagnetic Applications, vol. 16, no. 2, pp. 514-528, 2017.

[54] S. Ghosh, S. Bhattacharyya, D. Chaurasiya, and K. V. Srivastava, "An ultrawideband ultrathin metamaterial absorber based on circular split rings," IEEE antennas and wireless propagation letters, vol. 14, pp. 1172-1175, 2015.

[55] T. T. Nguyen and S. Lim, "Design of metamaterial absorber using eightresistive-arm cell for simultaneous broadband and wide-incidence-angle absorption," Scientific reports, vol. 8, 2018. 
[56] M. Yoo and S. Lim, "Polarization-independent and ultrawideband metamaterial absorber using a hexagonal artificial impedance surface and a resistorcapacitor layer," IEEE transactions on antennas and propagation, vol. 62, no. 5, pp. 2652-2658, 2014.

[57] A. P. Sohrab and Z. Atlasbaf, "A circuit analog absorber with optimum thickness and response in X-band," IEEE Antennas and Wireless Propagation Letters, vol. 12, pp. 276-279, 2013.

[58] M. D. Banadaki, A. A. Heidari, and M. Nakhkash, "A metamaterial absorber with a new compact unit cell," IEEE Antennas and Wireless Propagation Letters, vol. 17, no. 2, pp. 205-208, 2017.

[59] D. J. Cho, F. Wang, X. Zhang, and Y. R. Shen, "Contribution of the electric quadrupole resonance in optical metamaterials," Physical Review B, vol. 78, no. 12, p. 121101, 2008.

[60] Y. Cheng, H. Zou, J. Yang, X. Mao, and R. Gong, "Dual and broadband terahertz metamaterial absorber based on a compact resonator structure," Optical Materials Express, vol. 8, no. 10, pp. 3104-3114, 2018.

[61] N. T. Q. Hoa, T. S. Tuan, L. T. Hieu, and B. L. Giang, "Facile design of an ultra-thin broadband metamaterial absorber for C-band applications," Scientific reports, vol. 9, no. 1, p. 468, 2019.

[62] A. Sarkhel and S. R. B. Chaudhuri, "Compact quad-band polarizationinsensitive ultrathin metamaterial absorber with wide angle stability," IEEE Antennas and Wireless Propagation Letters, vol. 16, pp. 3240-3244, 2017.

[63] D. Lee, N. T. Trung, U.-C. Moon, and S. Lim, "Optimal parameter retrieval for metamaterial absorbers using the least-square method for wide incidence angle insensitivity," Applied optics, vol. 56, no. 16, pp. 4670-4674, 2017.

[64] J. Yang and Z. Shen, "A thin and broadband absorber using double-square loops," IEEE Antennas and Wireless Propagation Letters, vol. 6, pp. 388-391, 2007.

[65] E. K. Chong and S. H. Zak, An introduction to optimization, vol. 76. John Wiley \& Sons, 2013.

[66] D. M. Pozar, "RCS reduction for a microstrip antenna using a normally biased ferrite substrate," IEEE microwave and guided wave letters, vol. 2, no. 5, pp. 196-198, 1992. 
[67] J. Volakis, A. Alexanian, and J. Jin, "Broadband RCS reduction of rectangular patch by using distributed loading," Electronics Letters, vol. 28, no. 25, pp. 2322-2323, 1992.

[68] M. Gustafsson, "RCS reduction of integrated antenna arrays and radomes with resistive sheets," in 2006 IEEE Antennas and Propagation Society International Symposium, pp. 3479-3482, IEEE, 2006.

[69] J. Zhang, J. Wang, M. Chen, and Z. Zhang, "RCS reduction of patch array antenna by electromagnetic band-gap structure," IEEE Antennas and Wireless Propagation Letters, vol. 11, pp. 1048-1051, 2012.

[70] Y. Liu and X. Zhao, "Perfect absorber metamaterial for designing low-RCS patch antenna," IEEE Antennas and Wireless Propagation Letters, vol. 13, pp. 1473-1476, 2014.

[71] J. Ren, S. Gong, and W. Jiang, "Low-RCS monopolar patch antenna based on a dual-ring metamaterial absorber," IEEE Antennas and Wireless Propagation Letters, vol. 17, no. 1, pp. 102-105, 2018.

[72] T. Liu, X. Cao, J. Gao, Q. Zheng, W. Li, and H. Yang, "RCS reduction of waveguide slot antenna with metamaterial absorber," IEEE Transactions on Antennas and Propagation, vol. 61, no. 3, pp. 1479-1484, 2013.

[73] M. Jiang and L. Hanzo, "Multiuser MIMO-OFDM for next-generation wireless systems," Proceedings of the IEEE, vol. 95, no. 7, pp. 1430-1469, 2007.

[74] R. Zhang and C. K. Ho, "MIMO broadcasting for simultaneous wireless information and power transfer," IEEE Transactions on Wireless Communications, vol. 12, no. 5, pp. 1989-2001, 2013.

[75] S. J. Spiegel and I. I. Kovacs, "An efficient integration of GPS and WCDMA radio front-ends," IEEE Transactions on Microwave Theory and Techniques, vol. 52, no. 4, pp. 1125-1131, 2004.

[76] B. C. Pan, W. X. Tang, M. Q. Qi, H. F. Ma, Z. Tao, and T. J. Cui, "Reduction of the spatially mutual coupling between dual-polarized patch antennas using coupled metamaterial slabs," Scientific reports, vol. 6, p. 30288, 2016.

[77] G. Zhai, Z. N. Chen, and X. Qing, "Enhanced isolation of a closely spaced four-element MIMO antenna system using metamaterial mushroom," IEEE Transactions on Antennas and Propagation, vol. 63, no. 8, pp. 3362-3370, 2015. 
[78] F. Yang and Y. Rahmat-Samii, "Microstrip antennas integrated with electromagnetic band-gap (EBG) structures: A low mutual coupling design for array applications," IEEE transactions on antennas and propagation, vol. 51, no. 10, pp. 2936-2946, 2003.

[79] D. Guha, S. Biswas, M. Biswas, J. Y. Siddiqui, and Y. M. Antar, "Concentric ring-shaped defected ground structures for microstrip applications," IEEE Antennas and Wireless Propagation Letters, vol. 5, pp. 402-405, 2006.

[80] F.-G. Zhu, J.-D. Xu, and Q. Xu, "Reduction of mutual coupling between closely-packed antenna elements using defected ground structure," Electronics Letters, vol. 45, no. 12, pp. 601-602, 2009.

[81] Qualcomm, "5G spectrum access," 2017. https://www.itu.int/dms_pub/ itu-r/oth/0a/OE/ROAOE0000C60001PDFE. pdf, Last accessed on 2019-0712. 